ESAIM: M2AN 48 (2014) 815-858

DOI: $10.1051 / \mathrm{m} 2 \mathrm{an} / 2013122$
ESAIM: Mathematical Modelling and Numerical Analysis

www.esaim-m2an.org

\title{
MULTISCALE FINITE ELEMENT APPROACH FOR "WEAKLY" RANDOM PROBLEMS AND RELATED ISSUES
}

\author{
Claude Le Bris ${ }^{1,2}$, Frédéric Legoll $^{2,3}$ And Florian Thomines ${ }^{2,3}$
}

\begin{abstract}
We address multiscale elliptic problems with random coefficients that are a perturbation of multiscale deterministic problems. Our approach consists in taking benefit of the perturbative context to suitably modify the classical Finite Element basis into a deterministic multiscale Finite Element basis. The latter essentially shares the same approximation properties as a multiscale Finite Element basis directly generated on the random problem. The specific reference method that we use is the Multiscale Finite Element Method. Using numerical experiments, we demonstrate the efficiency of our approach and the computational speed-up with respect to a more standard approach. In the stationary setting, we provide a complete analysis of the approach, extending that available for the deterministic periodic setting.
\end{abstract}

Mathematics Subject Classification. 35B27, 65M60, 65M12, 35R60,60H.

Received November 7, 2011.

Published online April 8, 2014.

\section{Overview OF OUR APPROACH AND RESUlts}

The Multiscale Finite Element Method (henceforth abbreviated as MsFEM) is a popular numerical approach for multiscale problems (see [3,11,18,20,30-32,37-39]). It consists in a Galerkin approximation of the original problem over a finite dimensional space generated by basis functions that are specifically adapted to the problem under consideration.

This approach is popular for a twofold reason. First, its use is not restricted to multiscale problems that converge to a homogenized problem in the limit of vanishing ratio between the small scale and the macroscopic scale. It may be applied to much more general situations. Second, when the problem does converge to a homogenization problem, the MsFEM approach is meant to approximate the solution of the problem with the small scale $\varepsilon$ at its actual small value and not "only" in the asymptotic regime $\varepsilon \rightarrow 0$, which is the regime addressed by homogenization theory.

Keywords and phrases. Weakly stochastic homogenization, finite elements, Galerkin methods, highly oscillatory PDE.

1 CERMICS, École Nationale des Ponts et Chaussées, Université Paris-Est, 6 et 8 avenue Blaise Pascal, 77455 Marne-La-Vallée Cedex 2, France. lebris@cermics.enpc.fr

2 INRIA Rocquencourt, MICMAC team-project, Domaine de Voluceau, B.P. 105, 78153 Le Chesnay Cedex, France.

3 Laboratoire Navier, École Nationale des Ponts et Chaussées, Université Paris-Est, 6 et 8 avenue Blaise Pascal, 77455 Marne-

La-Vallée Cedex 2, France. legoll@lami.enpc.fr; thominef@lami.enpc.fr 
To fix the ideas, consider the problem of finding $u^{\varepsilon}$ solving

$$
-\operatorname{div}\left[A^{\varepsilon} \nabla u^{\varepsilon}\right]=f \quad \text { in } \mathcal{D}, \quad u^{\varepsilon}=0 \text { on } \partial \mathcal{D},
$$

on a bounded domain $\mathcal{D} \subset \mathbb{R}^{d}$, with $f \in L^{2}(\mathcal{D})$, and where $A^{\varepsilon}$ is a uniformly bounded, coercive matrix that varies at scale $\varepsilon$. A standard Finite Element Method (FEM) would require a space discretization of the domain at the scale $\varepsilon$ in order to capture the oscillations of $u^{\varepsilon}$ at scale $\varepsilon$. This is prohibitively expensive. The MsFEM aims at accurately approximating $u^{\varepsilon}$ using a limited number of degrees of freedom. It does not require the matrix $A^{\varepsilon}$ to be periodic (namely $A^{\varepsilon}(x)=A_{\text {per }}(x / \varepsilon)$ for a fixed periodic matrix $A_{\text {per}}$ ) or stationary.

We now briefly describe the approach and present the aim of this article. Starting from a coarse mesh $\mathcal{T}_{h}$ with a standard (say $\left.\mathbb{P}_{1}\right)$ Finite Element basis set of functions $\left\{\phi_{i}^{0}\right\}_{i=1}^{L}$, generating the associated space

$$
\mathcal{V}_{h}:=\operatorname{span}\left(\phi_{i}^{0}, i=1, \ldots, L\right),
$$

we first numerically build the MsFEM basis functions $\phi_{i}^{\varepsilon}$. Several definitions of these basis functions have been proposed in the literature (yielding different numerical methods), and we detail this in the sequel (see e.g. (2.7)(2.8)-(2.9)). For the moment, it is sufficient to know that, to each $\phi_{i}^{0}$, which varies at the macroscopic scale, is associated a function $\phi_{i}^{\varepsilon}$, with variations at the scale $\varepsilon$. In practice, $\phi_{i}^{\varepsilon}$ is numerically computed (in fact, pre-computed), using the specificities of the problem addressed. These highly oscillatory functions $\phi_{i}^{\varepsilon}$ generate the finite dimensional space

$$
\mathcal{W}_{h}:=\operatorname{span}\left(\phi_{i}^{\varepsilon}, i=1, \ldots, L\right) .
$$

Note that $\mathcal{W}_{h}$ and $\mathcal{V}_{h}$ share the same dimension.

We next define the MsFEM solution $u_{M}$ using a Galerkin approximation of (1.1) on $\mathcal{W}_{h}$, instead of $\mathcal{V}_{h}$. Again, details will be given below. The MsFEM solution $u_{M}$ provided by the approach reads

$$
u_{M}(x)=\sum_{i=1}^{L}\left(U_{M}\right)_{i} \phi_{i}^{\varepsilon}(x),
$$

for some coefficients $\left\{\left(U_{M}\right)_{i}\right\}_{i=1}^{L}$. Of course, these coefficients depend on $\varepsilon$, but this dependency is kept implicit in the sequel.

We now turn our attention to the stochastic problem

$$
-\operatorname{div}\left[A^{\varepsilon}(\cdot, \omega) \nabla u^{\varepsilon}(\cdot, \omega)\right]=f \text { in } \mathcal{D}, \quad u^{\varepsilon}(\cdot, \omega)=0 \text { on } \partial \mathcal{D},
$$

and a typical quantity of interest $\mathbb{E}\left[u^{\varepsilon}(x, \cdot)\right]$, which is traditionally approximated using a Monte Carlo method. Introducing a set of $\mathcal{M}$ realizations of the stochastic matrix $\left\{A^{\varepsilon, m}\right\}_{1 \leq m \leq \mathcal{M}}$, a direct, naïve application of the MsFEM paradigm would consist in first computing for each realization $m$ the stochastic MsFEM basis functions $\phi_{i}^{\varepsilon, m}(x, \omega)$, next performing a Galerkin approximation of (1.2) using this MsFEM basis set to compute $\left\{u_{M}^{m}(x, \omega)\right\}_{1 \leq m \leq \mathcal{M}}$, and eventually approximating $\mathbb{E}\left[u^{\varepsilon}(x, \cdot)\right]$ by

$$
\mathbb{E}\left[u^{\varepsilon}(x, \cdot)\right] \approx \frac{1}{\mathcal{M}} \sum_{m=1}^{\mathcal{M}} u_{M}^{m}(x, \omega) .
$$

Such an approach is unpractical because of the prohibitively expensive computational load.

To reduce the computational cost and make the MsFEM approach practical in such a stochastic context, a natural idea we investigate in this article is to consider a less generic setting, for which a dedicated, more computationally affordable approach, can be designed. One possibility is to consider matrices $A^{\varepsilon}(x, \omega) \equiv A^{\varepsilon}(x)+$ $B(x, \omega)$ in $(1.2)$ that are not highly oscillatory in their stochastic part. In such cases, dedicated approaches have been proposed, we refer to [35] for more details. Another approach is to reduce the number of Monte-Carlo 
simulations used for the computation of the multiscale basis functions. In $[1,26]$, the authors assume that their coefficient can be written as a Karhunen-Loève type expansion, and apply a collocation method to a priori choose some sparse realizations for which they compute the multiscale basis functions.

In this article, we consider one of the many alternate variants of problem (1.2). We suppose that $A^{\varepsilon}(x, \omega)$ is highly oscillatory in both its deterministic and stochastic components, but that it is a perturbation of a deterministic matrix. More precisely, we assume that

$$
A^{\varepsilon}(x, \omega) \equiv A_{\eta}^{\varepsilon}(x, \omega)=A_{0}^{\varepsilon}(x)+\eta A_{1}^{\varepsilon}(x, \omega),
$$

where $A_{0}^{\varepsilon}$ is a deterministic matrix and $\eta$ is a small deterministic parameter. This model may be well suited for heterogeneous materials (or, more generally, media) that, although not periodic, are not fully stochastic, in the sense that they may be considered as a perturbation of a deterministic material. We call this setting the weakly stochastic setting. Note that many practical situations, involving actual materials or media, can be considered, at a good level of approximation, as perturbations of a deterministic (often periodic) setting (see e.g. [41]).

In a series of recent works (see $[14,15,25]$ and [6-8]; see also [5] for a unified presentation), we have considered such a setting, in the context of homogenization theory (the matrix $A_{\eta}^{\varepsilon}(x, \omega)$ in $(1.2)-(1.3)$ reads $A_{\eta}^{\varepsilon}(x, \omega)=$ $A_{\eta}(x / \varepsilon, \omega)$ for a stationary matrix $A_{\eta}(x, \omega)$, which is, in a sense to be made precise, a perturbation of a periodic matrix). We have shown there that, in such a case, the workload for computing the homogenized solution is significantly lighter than for generic stochastic homogenization, and actually comparable to the workload for periodic homogenization. We will show in the sequel that the MsFEM can be adapted to this weakly stochastic setting, providing an approximation of the solution $u_{\eta}^{\varepsilon}$ to (1.2)-(1.3), for fixed $\varepsilon$, at a much smaller computational cost than the direct approach.

The main idea of our proposed approach is to compute a set of deterministic MsFEM basis functions using $A_{0}^{\varepsilon}$, the deterministic part of $A_{\eta}^{\varepsilon}$ in the expansion (1.3), and then to perform Monte Carlo realizations at the macroscale level using a set of $\mathcal{M}$ realizations of the random matrix $\left\{A_{\eta}^{\varepsilon, m}(x, \omega)\right\}_{1 \leq m \leq \mathcal{M}}$ (see Sect. 2 for a detailed presentation). Note that, for each of these realizations, we solve the original problem, with the complete matrix $A_{\eta}^{\varepsilon}$, and not only its deterministic part. Only the basis set is taken deterministic. By construction, the approach provides an approximation

$$
u_{S}(x, \omega)=\sum_{i=1}^{L}\left(U_{S}(\omega)\right)_{i} \phi_{i}^{\varepsilon}(x)
$$

of $u_{\eta}^{\varepsilon}(x, \omega)$, where the basis functions $\phi_{i}^{\varepsilon}$ are deterministic. These basis functions are computed only once, hence the cost to compute $\left\{u_{S}^{m}(x, \omega)\right\}_{1 \leq m \leq \mathcal{M}}$ is much smaller than the cost to compute $\left\{u_{M}^{m}(x, \omega)\right\}_{1 \leq m \leq \mathcal{M}}$. This is especially true if (1.2) has to be solved for many right-hand sides $f$. We expect that this approximation $u_{S}$ is as accurate as $u_{M}$ for small $\eta$. We show below that this is indeed the case, when $A_{\eta}^{\varepsilon}$ is a perturbation of $A_{0}^{\varepsilon}$ (see Sect. 3 for numerical tests).

We would like to note that the MsFEM is not the only multiscale technique based on finite elements. The bottom line of our approach, consisting of generating suitable multiscale functions for the discretization of a weakly stochastic problem, using for this purpose the deterministic reference problem, can in principle be applied to other multiscale techniques. Another popular technique is the HMM method [27-29], for which our approach could in principle be easily adapted.

In the numerical tests reported on in Section 3, we compare, in the $H^{1}$ norm, $u_{\eta}^{\varepsilon}$ (the exact solution to (1.2) with the matrix $A^{\varepsilon} \equiv A_{\eta}^{\varepsilon}$ given by (1.3)) with $u_{S}$ (the solution provided by our approach) and $u_{M}$ (the solution provided by the ideal, expensive approach). The quantity $\left\|u_{\eta}^{\varepsilon}-u_{M}\right\|_{H^{1}(\mathcal{D})}$ represents the best possible accuracy that we can achieve, in the sense that our approach inherits the limitations of the MsFEM approach. We thus cannot expect our approximation $u_{S}$ to be more accurate than $u_{M}$. We can only hope to compute an approximation of comparable quality with a much reduced workload. The numerical results we obtain confirm that, for small $\eta$ in (1.3), the quantity $\left\|u_{S}-u_{\eta}^{\varepsilon}\right\|_{H^{1}(\mathcal{D})}$ is of the same order of magnitude as $\left\|u_{M}-u_{\eta}^{\varepsilon}\right\|_{H^{1}(\mathcal{D})}$, 
although, we repeat it, the computational cost to compute $u_{S}$ is much smaller than that to compute $u_{M}$. In Section 3, we also show the advantage of performing Monte Carlo realizations at the macroscale level (using the random matrix $A_{\eta}^{\varepsilon, m}$ ) over solving a deterministic macroscale problem with only the deterministic part of $A_{\eta}^{\varepsilon}$.

We next derive error bounds for our approach in Section 4. We recall that, in the deterministic setting, a classical context for proving convergence of the MsFEM approach is the case when, in the reference problem (1.1), the matrix reads $A^{\varepsilon}(x)=A_{\text {per }}\left(\frac{x}{\varepsilon}\right)$ for a fixed periodic matrix $A_{\text {per. }}$ Likewise, to be able to perform our theoretical analysis in the stochastic setting, we assume in Section 4 that $A_{\eta}^{\varepsilon}(x, \omega)=A_{\eta}\left(\frac{x}{\varepsilon}, \omega\right)$ for a fixed stationary random matrix $A_{\eta}$. The problem (1.2)-(1.3) then admits a homogenized limit when $\varepsilon$ vanishes.

Our proof follows the same lines as that in the deterministic setting, which we now briefly review (see the introduction of Sect. 4 for more details on the structure of the proof). The MsFEM is a Galerkin approximation, that we assume momentarily, for the sake of clarity, to be a conforming approximation (this is indeed the case when, for defining the highly oscillatory basis functions $\phi_{i}^{\varepsilon}$, oversampling is not used). The error is then estimated using the Céa lemma:

$$
\left\|u^{\varepsilon}-u_{M}\right\|_{H^{1}(\mathcal{D})} \leq C \inf _{v_{h} \in \mathcal{W}_{h}}\left\|u^{\varepsilon}-v_{h}\right\|_{H^{1}(\mathcal{D})},
$$

where $u^{\varepsilon}$ is the solution to the reference deterministic highly oscillatory problem (1.1), $u_{M}$ is the MsFEM solution and $C$ is a constant independent of $\varepsilon$ and $h$. Taking advantage of the homogenization setting, we introduce the two-scale expansion

$$
v^{\varepsilon}=u^{\star}+\varepsilon \sum_{i=1}^{d} w_{e_{i}}^{0}\left(\frac{\dot{z}}{\varepsilon}\right) \partial_{i} u^{\star}
$$

of $u^{\varepsilon}$, where $u^{\star}$ is the homogenized solution, $w_{e_{i}}^{0}$ is the periodic corrector associated to $e_{i} \in \mathbb{R}^{d}$, and $\partial_{i} u^{\star}$ denotes the partial derivative $\frac{\partial u^{\star}}{\partial x_{i}}$. We next write

$$
\left\|u^{\varepsilon}-u_{M}\right\|_{H^{1}(\mathcal{D})} \leq C\left(\left\|u^{\varepsilon}-v^{\varepsilon}\right\|_{H^{1}(\mathcal{D})}+\inf _{v_{h} \in \mathcal{W}_{h}}\left\|v^{\varepsilon}-v_{h}\right\|_{H^{1}(\mathcal{D})}\right)
$$

The first term in the above right-hand side is estimated using standard homogenization results on the rate of convergence of $v^{\varepsilon}-u^{\varepsilon}$. To estimate the second term, one considers a suitably chosen element $v_{h} \in \mathcal{W}_{h}$, for which $\left\|v^{\varepsilon}-v_{h}\right\|_{H^{1}(\mathcal{D})}$ can be directly bounded.

Following the same strategy in our stochastic setting, we estimate the distance between the solution $u_{\eta}^{\varepsilon}$ to the reference stochastic problem (1.2)-(1.3) and the weakly stochastic MsFEM solution $u_{S}$ as

$$
\left\|u_{\eta}^{\varepsilon}(\cdot, \omega)-u_{S}(\cdot, \omega)\right\|_{H^{1}(\mathcal{D})} \leq C\left(\left\|u_{\eta}^{\varepsilon}(\cdot, \omega)-v_{\eta}^{\varepsilon}(\cdot, \omega)\right\|_{H^{1}(\mathcal{D})}+\inf _{v_{h} \in \mathcal{W}_{h}}\left\|v_{\eta}^{\varepsilon}(\cdot, \omega)-v_{h}\right\|_{H^{1}(\mathcal{D})}\right) .
$$

We observe that a key ingredient for the proof is the rate of convergence of the difference between the reference solution $u_{\eta}^{\varepsilon}$ and its two-scale expansion $v_{\eta}^{\varepsilon}$. Such a result is classical in periodic homogenization, but, to the best of our knowledge, open in the general stationary case (in dimensions higher than one). One only knows that $u_{\eta}^{\varepsilon}-v_{\eta}^{\varepsilon}$ vanishes (in some appropriate norm) when $\varepsilon \rightarrow 0$. However, in the particular case when $A_{\eta}^{\varepsilon}$ is only weakly stochastic, it is possible to obtain such a result, as we have shown in [42]. Hence, exploiting the specificity of our weakly stochastic setting, we are able to obtain (see our main result, Thm. 4.5 and estimate (4.37)):

$$
\sqrt{\mathbb{E}\left[\left\|u_{\eta}^{\varepsilon}-u_{S}\right\|_{H_{h}^{1}}^{2}\right]} \leq C\left(\sqrt{\varepsilon}+h+\frac{\varepsilon}{h}+\eta\left(\frac{\varepsilon}{h}\right)^{d / 2} \ln (N(h))+\eta+\eta^{2} \mathcal{C}(\eta)\right),
$$

where $\|\cdot\|_{H_{h}^{1}}$ is a broken $H^{1}$ norm, $C$ is a constant independent of $\varepsilon, h$ and $\eta, \mathcal{C}$ is a bounded function as $\eta$ goes to 0 , and $N(h)$ is the number of elements in the mesh (roughly of order $h^{-d}$ in dimension $d$ ). As is often the 
case in the deterministic setting, we use here (both for our numerical tests and in the analysis) the oversampling technique. Consequently, the basis functions $\phi_{i}^{\varepsilon}$ do not belong to $H_{0}^{1}(\mathcal{D})$, hence the use of a broken $H^{1}$ norm in the above estimate. As we point out below, when $\eta=0$ in (1.3), our approach reduces to the standard deterministic MsFEM (with oversampling), and the above estimates then agree with those proved in [32].

This article is organized as follows. First, in Section 2, we describe the MsFEM approach. For consistency, we begin by the deterministic setting in Section 2.1, and point out there that the direct adaptation to the general stochastic setting yields a prohibitively expensive approach. The adaptation of the approach to the weakly stochastic setting is described in Section 2.2. We next turn to numerical simulations, in Section 3. Some procedures to efficiently implement the approach are first described in Section 3.1. We next consider a onedimensional test (see Sect. 3.2), which is useful for several reasons. First, it allows to calibrate some numerical parameters, such as the number $M$ of independent realizations when estimating the exact expectation by an empirical mean. Second, we assess the accuracy of our approach with respect to the magnitude of $\eta$. We demonstrate there that $\eta$ does not have to be extremely small for our method to be very efficient. For instance, on the test case considered in Section 3.2, we show that our approach is as accurate as the expensive, direct approach as soon as $\eta$ is such that

$$
\left\|\frac{\eta a_{1}^{\varepsilon}}{a_{0}^{\varepsilon}}\right\|_{L^{\infty}(\mathbb{R} \times \Omega)} \text { is equal to or smaller than 0.1, }
$$

where $a_{0}^{\varepsilon}$ is the deterministic component of the diffusion coefficient $a_{\eta}^{\varepsilon}$ and $\eta a_{1}^{\varepsilon}$ is the stochastic component (see expansion (1.3)). On the other hand, as pointed out above, our approach is not meant to address the regime when $\eta \approx 1$. Lastly, we also assess the accuracy of our approach with respect to the presence of frequencies in the random coefficient $a_{\eta}^{\varepsilon}$ that are not taken into account in the MsFEM basis set. We next turn to two test cases in dimension two, where we observe that our approach performs as well as in the one-dimensional case (see Sect. 3.3). In particular, in Section 3.3.2, we successfully address a classical test-case of the literature. In Section 3.4, we compare our approach with a fully deterministic approach. All the information about variance is lost when using the latter approach. In contrast, using our approach, we show that we can accurately approximate some quantities of interest which are random in nature, such as the variance of the solution.

Section 4 is devoted to the analysis of the approach, in the homogenization setting (i.e. when the matrix in (1.2) reads $A^{\varepsilon}(x, \omega)=A\left(\frac{x}{\varepsilon}, \omega\right)$ where $A$ is stationary). Our main result, Theorem 4.5 , is presented in Section 4.1, and proved in Section 4.2. The proofs of some technical results are collected in Appendix A. In addition, we specifically consider the one dimensional case in Section 4.3.

\section{MSFEM-TYPE APPROACHES}

For consistency and the convenience of the reader, we present in this section the MsFEM approach to solve the original elliptic problem (1.1). For clarity, we begin by presenting the approach in a deterministic setting. The reader familiar with the MsFEM may easily skip this section and directly proceed to Section 2.2, where we present our approach in a weakly stochastic setting.

\subsection{Description in a classical deterministic setting}

Let $u^{\varepsilon} \in H^{1}(\mathcal{D})$ be the solution to (1.1), where the matrix $A^{\varepsilon} \in\left(L^{\infty}(\mathcal{D})\right)^{d \times d}$ satisfies the standard coercivity condition: there exists two constants $a_{+} \geq a_{-}>0$ such that, for any $\varepsilon$,

$$
\forall \xi \in \mathbb{R}^{d}, \quad a_{-}|\xi|^{2} \leq \xi^{T} A^{\varepsilon}(x) \xi \text { a.e. in } \mathcal{D} \quad \text { and } \quad\left\|A^{\varepsilon}\right\|_{L^{\infty}(\mathcal{D})} \leq a_{+} .
$$

Note that the MsFEM approach is not restricted to the periodic setting. We therefore do not assume that $A^{\varepsilon}(x)=A_{\text {per }}(x / \varepsilon)$ for a fixed periodic matrix $A_{\text {per }}$. 
The MsFEM approach consists in performing a variational approximation of (1.1) where the basis functions are precomputed and encode the fast oscillations present in (1.1). In the sequel we argue on the following formulation, equivalent to (1.1):

$$
\text { Find } u^{\varepsilon} \in H_{0}^{1}(\mathcal{D}) \text { such that, for any } v \in H_{0}^{1}(\mathcal{D}), \quad \mathcal{A}_{\varepsilon}\left(u^{\varepsilon}, v\right)=b(v),
$$

where

$$
\mathcal{A}_{\varepsilon}(u, v)=\int_{\mathcal{D}}(\nabla v(x))^{T} A^{\varepsilon}(x) \nabla u(x) \mathrm{d} x \text { and } b(v)=\int_{\mathcal{D}} f(x) v(x) \mathrm{d} x .
$$

The MsFEM is a three-step approach:

1. introduce a standard discretization of the domain $\mathcal{D}$ using a coarse mesh as compared to the small scale oscillations of $A^{\varepsilon}$;

2. for each element $\mathbf{K}$ of the coarse mesh, compute the basis function $\phi_{i}^{\varepsilon, \mathbf{K}}$ as the solution of an elliptic equation posed in $\mathbf{K}$ (see e.g. (2.7)-(2.8)-(2.9) below);

3. solve the Galerkin approximation of (2.1), for the set of basis functions defined at Step 2.

The advantage of the approach is that, for the same accuracy of the approximation as that provided by a standard FEM, the macroscale mesh can be chosen sufficiently coarse so that the resulting discretized problem has a limited number of degrees of freedom, and may thus be computationally solved inexpensively. This is observed in practice [37], and proven by a theoretical analysis (see [32,38]) when the problem $(2.1)$ admits a homogenized limit. See also [30] and references therein.

To further illustrate this fact, we reproduce here a simple one-dimensional analysis we borrow from A. Lozinski (see [44], Chap. 6 and [17]). This analysis explains remarkably well the interest of the approach, and, in contrast to $[32,38]$, is not restricted to a homogenization setting. Consider the one-dimensional domain $\mathcal{D}=(0,1)$ and the reference problem

$$
\mathcal{L} u=f, \quad u(0)=u(1)=0,
$$

for the operator $\mathcal{L} u:=-\left(\nu u^{\prime}\right)^{\prime}$, where $f \in L^{2}(0,1)$ and $\nu \in L^{\infty}(0,1)$ with $\nu(x) \geq \nu_{\min }>0$ almost everywhere on $(0,1)$. The function $\nu$ may have oscillations at a small scale. The associated weak formulation reads

$$
\text { Find } u \in H_{0}^{1}(0,1) \text { such that, for any } v \in H_{0}^{1}(0,1), \quad a(u, v)=b(v) \text {, }
$$

with

$$
a(u, v)=\int_{0}^{1} \nu(x) u^{\prime}(x) v^{\prime}(x) \mathrm{d} x \quad \text { and } \quad b(v)=\int_{0}^{1} f(x) v(x) \mathrm{d} x .
$$

We now introduce the nodes $0=x_{0}<x_{1}<\cdots<x_{L}=1$ that define the elements $K_{i}=\left[x_{i-1}, x_{i}\right]$. Let $h=\max \left|x_{i}-x_{i-1}\right|$ be the mesh size. The multiscale finite element space

$$
\mathcal{W}_{h}=\left\{v_{h} \in C^{0}(0,1) \text { such that } \mathcal{L} v_{h}=0 \text { on each } K_{i}\right\},
$$

defined using the operator $\mathcal{L}$, is adapted to the problem under study. We next proceed with a Galerkin approximation of $(2.2)$ using the space $\mathcal{W}_{h}$ :

$$
\text { Find } u_{h} \in \mathcal{W}_{h} \text { such that, for any } v_{h} \in \mathcal{W}_{h}, \quad a\left(u_{h}, v_{h}\right)=b\left(v_{h}\right) .
$$

The solution $u_{h}$ then satisfies

$$
\left\|u-u_{h}\right\|_{E} \leq \frac{h}{\pi \sqrt{\nu_{\text {min }}}}\|f\|_{L^{2}(0,1)}
$$

where $\|\cdot\|_{E}=\sqrt{a(\cdot, \cdot)}$ is the energy norm. The proof of this estimate goes as follows. By definition of $u$ and $u_{h}$, we have $a\left(u-u_{h}, v_{h}\right)=0$ for any $v_{h} \in \mathcal{W}_{h}$. Hence, $u_{h}$ is the orthogonal projection of $u$ on $\mathcal{W}_{h}$ according to the scalar product $a(\cdot, \cdot)$. Since $\|\cdot\|_{E}$ is the norm associated to that scalar product, we have

$$
\left\|u-u_{h}\right\|_{E}=\inf _{v_{h} \in \mathcal{W}_{h}}\left\|u-v_{h}\right\|_{E} .
$$


Choose $v_{h}$ to be the finite element interpolant of $u$, which is defined by $v_{h}\left(x_{i}\right)=u\left(x_{i}\right)$ for any $i=0,1, \ldots, L$, and consider the interpolation error $e=u-v_{h}$. On each element $K_{i}$, we have, precisely because the space $\mathcal{W}_{h}$ is defined as (2.3),

$$
\mathcal{L} e=-\left(\nu e^{\prime}\right)^{\prime}=f \quad \text { with } \quad e\left(x_{i-1}\right)=e\left(x_{i}\right)=0 .
$$

We multiply by $e$, integrate by part and obtain

$$
\int_{x_{i-1}}^{x_{i}} \nu(x)\left|e^{\prime}(x)\right|^{2} \mathrm{~d} x=\int_{x_{i-1}}^{x_{i}} f(x) e(x) \mathrm{d} x \leq\|f\|_{L^{2}\left(K_{i}\right)}\|e\|_{L^{2}\left(K_{i}\right)} .
$$

Since $e$ vanishes on the boundary of $K_{i}$, the Poincaré inequality with the best constant $\left(x_{i}-x_{i-1}\right) / \pi$ yields

$$
\|e\|_{L^{2}\left(K_{i}\right)} \leq \frac{x_{i}-x_{i-1}}{\pi}\left\|e^{\prime}\right\|_{L^{2}\left(K_{i}\right)} \leq \frac{h}{\pi \sqrt{\nu_{\min }}}\left(\int_{x_{i-1}}^{x_{i}} \nu(x)\left|e^{\prime}(x)\right|^{2} \mathrm{~d} x\right)^{1 / 2} .
$$

By substitution in (2.6), we obtain

$$
\int_{x_{i-1}}^{x_{i}} \nu(x)\left|e^{\prime}(x)\right|^{2} \mathrm{~d} x \leq \frac{h^{2}}{\pi^{2} \nu_{\min }}\|f\|_{L^{2}\left(K_{i}\right)}^{2} .
$$

Summing over the elements and using (2.5) yields (2.4). Using again that $\nu$ is bounded from below, we deduce from (2.4) that

$$
\left\|u-u_{h}\right\|_{H^{1}(0,1)} \leq \frac{h}{C_{\mathcal{D}} \pi \nu_{\min }}\|f\|_{L^{2}(0,1)},
$$

where $C_{\mathcal{D}}$ is the Poincaré constant of the domain $\mathcal{D}=(0,1)$. As pointed out in Chapter 6 of [44], the interest of the above estimate (or of estimate (2.4)) lies in the fact that the constant in the right-hand side only depends on $\nu$ through $\nu_{\text {min }}$, and remains the same even if $\nu$ oscillates at a small scale. In contrast, for a standard finite element method, the error is also proportional to $h$, but with a constant that depends on the $H^{2}$ norm of the exact solution $u$. With a standard finite element space $\mathcal{W}_{h}$, we indeed classically deduce by Céa's lemma that

$$
\left\|u-u_{h}\right\|_{H^{1}(0,1)} \leq \frac{\|\nu\|_{L^{\infty}(0,1)}}{C_{\mathcal{D}} \nu_{\min }} \inf _{v_{h} \in \mathcal{W}_{h}}\left\|u-v_{h}\right\|_{H^{1}(0,1)}=\frac{\|\nu\|_{L^{\infty}(0,1)}}{C_{\mathcal{D}} \nu_{\min }}\left\|u-R_{h} u\right\|_{H^{1}(0,1)}
$$

where $C_{\mathcal{D}}$ is the Poincaré constant of the domain $\mathcal{D}=(0,1)$, and $R_{h} u$ is the projection of $u$ on $\mathcal{W}_{h}$ according to the $H^{1}$ scalar product. We thus obtain that

$$
\left\|u-u_{h}\right\|_{H^{1}(0,1)} \leq C h \frac{\|\nu\|_{L^{\infty}(0,1)}}{\nu_{\min }}\left\|u^{\prime \prime}\right\|_{L^{2}(0,1)},
$$

where $C$ is independent from the functions $\nu$ and $u$. If $\nu$ oscillates at a small scale $(e . g . \nu(x)=\bar{\nu}(x / \varepsilon)$ for a fixed function $\bar{\nu}$ ), the $H^{2}$ norm of $u$ may be large (of the order of $\varepsilon^{-1}$ ). A FEM approach then requires $h$ to be smaller than $\varepsilon$ to reach a good accuracy.

We conclude this illustration by noting that such a general analysis of the MsFEM approach is not available in dimension $d \geq 2$. The analysis presented in [32,38], which is performed without any restriction on the dimension, additionally assumes that the matrix $A^{\varepsilon}$ in $(2.1)$ reads $A^{\varepsilon}(x)=A_{\text {per }}(x / \varepsilon)$ for a fixed periodic matrix $A_{\text {per }}$.

We now describe the MsFEM in a multidimensional setting.

\subsubsection{Definition of the coarse mesh}

For simplicity (see Rem. 2.1 below), we consider a classical $\mathbb{P}_{1}$ discretization of the domain $\mathcal{D}$. We denote by $\mathcal{T}_{h}$ the corresponding mesh, with $L$ nodes. Let $\phi_{i}^{0}, i=1, \ldots, L$, be the basis functions. We introduce the finite element space

$$
\mathcal{V}_{h}:=\operatorname{span}\left(\phi_{i}^{0}, i=1, \ldots, L\right),
$$




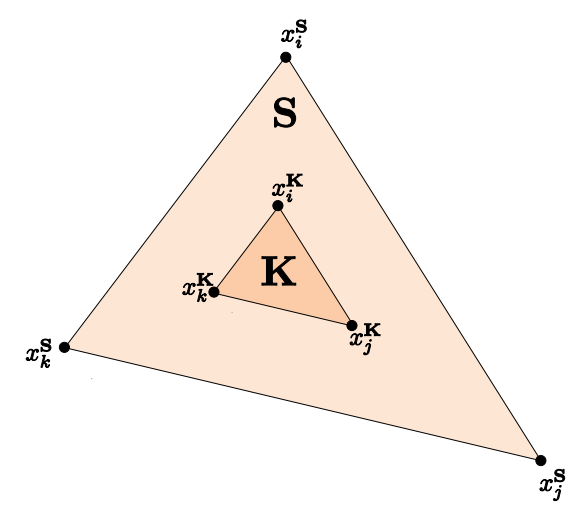

Figure 1. Definition of $\mathbf{S}$ (in 2D for clarity).

and define the restriction

$$
\phi_{i}^{0, \mathbf{K}}:=\left.\phi_{i}^{0}\right|_{\mathbf{K}}
$$

of these functions in each element $\mathbf{K}$.

Remark 2.1. We refer to [3] for a presentation of a MsFEM method that uses $\mathbb{P}_{2}$ macroscale basis functions.

\subsubsection{Definition of the MsFEM basis}

Several definitions of the MsFEM basis functions have been proposed in the literature (see e.g. $[3,30,32,37-39])$. They all follow the same pattern but they give rise to various methods. We present in the following the particular method that we have implemented. It makes use of the oversampling technique introduced in [37] and developed in [36].

For any element $\mathbf{K}$, we consider a domain $\mathbf{S} \supset \mathbf{K}$ (see Fig. 1), obtained from $\mathbf{K}$ by an homothetic transformation of center the centroid of $\mathbf{K}$, and of ratio larger than 1 .

Let $x_{j}^{\mathbf{S}}$ denote the coordinate of the vertex $j$ of the domain $\mathbf{S}$. For any vertex $i$ of $\mathbf{S}$, we introduce the affine function $\chi_{i}^{0, \mathbf{S}}$ (defined on $\mathbf{S}$ ) that satisfies the condition $\chi_{i}^{0, \mathbf{S}}\left(x_{j}^{\mathbf{S}}\right)=\delta_{i j}$ for all $j$. Let $\chi_{i}^{\varepsilon, \mathbf{S}} \in H^{1}(\mathbf{S})$ be the unique solution to the problem

$$
-\operatorname{div}\left[A^{\varepsilon}(x) \nabla \chi_{i}^{\varepsilon, \mathbf{S}}(x)\right]=0 \quad \text { in } \mathbf{S}, \quad \chi_{i}^{\varepsilon, \mathbf{S}}=\chi_{i}^{0, \mathbf{S}} \text { on } \partial \mathbf{S},
$$

which, in practice, is numerically solved e.g. using a finite element method with a mesh size adapted to the small scale $\varepsilon$. We then define the local basis functions

$$
\phi_{i}^{\varepsilon, \mathbf{K}}=\left.\sum_{j=1}^{d+1} \alpha_{i j} \chi_{j}^{\varepsilon, \mathbf{S}}\right|_{\mathbf{K}}
$$

as linear combinations of the restrictions of $\chi_{i}^{\varepsilon, \mathbf{S}}$ on $\mathbf{K}$, with $\alpha_{i j}$ chosen such that

$$
\forall 1 \leq i, j \leq d+1, \quad \phi_{i}^{0, \mathbf{K}}\left(x_{j}^{\mathbf{K}}\right)=\sum_{j=1}^{d+1} \alpha_{i j} \chi_{j}^{0, \mathbf{S}}\left(x_{j}^{\mathbf{K}}\right)=\delta_{i j},
$$

where $x_{j}^{\mathbf{K}}$ denotes the coordinate of the $j$ th vertex of the element $\mathbf{K}$. Note that the condition (2.9) is enforced on the function $\phi_{i}^{0, \mathbf{K}}$, and not on $\phi_{i}^{\varepsilon, \mathbf{K}}$. The coefficients $\alpha_{i j}$ are consequently independent from $\varepsilon$. As $\phi_{i}^{0, \mathbf{K}}$ and 
$\left.\chi_{j}^{0, \mathbf{S}}\right|_{\mathbf{K}}$ are both affine on $\mathbf{K}$, condition (2.9) implies that

$$
\forall 1 \leq i \leq d+1, \quad \forall x \in \mathbf{K}, \quad \phi_{i}^{0, \mathbf{K}}(x)=\sum_{j=1}^{d+1} \alpha_{i j} \chi_{j}^{0, \mathbf{S}}(x) .
$$

We next introduce the functions $\phi_{i}^{\varepsilon}$ defined on $\mathcal{D}$ by $\left.\phi_{i}^{\varepsilon}\right|_{\mathbf{K}}=\phi_{i}^{\varepsilon, \mathbf{K}}$ for all elements $\mathbf{K}$.

Note that the problems (2.7), indexed by $\mathbf{S}$, are all independent from one another. They may be solved in parallel.

\subsubsection{Macroscale problem}

We now introduce the finite dimensional space

$$
\mathcal{W}_{h}:=\operatorname{span}\left(\phi_{i}^{\varepsilon}, i=1, \ldots, L\right),
$$

and proceed with the approximation

$$
\text { Find } u_{M} \in \mathcal{W}_{h} \text { such that, for any } v \in \mathcal{W}_{h}, \quad \mathcal{A}_{\varepsilon}^{h}\left(u_{M}, v\right)=b(v),
$$

of (2.1), where

$$
\mathcal{A}_{\varepsilon}^{h}(u, v)=\sum_{\mathbf{K}} \int_{\mathbf{K}}(\nabla v(x))^{T} A^{\varepsilon}(x) \nabla u(x) \mathrm{d} x \text { and } b(v)=\int_{\mathcal{D}} f(x) v(x) \mathrm{d} x .
$$

Observe that $\phi_{i}^{\varepsilon}$ has jumps across the edges of the triangulation (due to the use of the oversampling technique), hence $\mathcal{W}_{h} \not \subset H^{1}(\mathcal{D})$, thus the broken integral used to define $\mathcal{A}_{\varepsilon}^{h}(u, v)$. On the other hand, since $\mathcal{W}_{h} \subset L^{2}(\mathcal{D})$, the linear form $b$ is well defined for $v \in \mathcal{W}_{h}$. The formulation (2.11) is a non-conforming Galerkin approximation of (2.1). This brings additional error terms in the error estimation (see Lem. 4.7 in Section 4). On another note, remark that the dimension of $\mathcal{W}_{h}$ is equal to $L$. The formulation (2.11) hence requires solving a linear system with only a limited number of degrees of freedom.

We are now in position to substantiate our claim in the introduction, where we briefly mentioned that, in the stochastic setting, a direct application of the MsFEM to approximate the solution to (1.2) is unpractical. It would indeed lead to compute, for each realization of $A^{\varepsilon}(x, \omega)$, first a basis set and second a macroscale solution. This approach has been briefly examined theoretically in [21]. It is prohibitively expensive. We therefore turn to an alternate approach.

\subsection{A weakly stochastic setting}

We now restrict the general setting and propose a dedicated, practical MsFEM type approach. Following up on previous works (see $[5,13,24,41]$ ) and as announced in (1.3), we assume here that the random matrix $A^{\varepsilon}(x, \omega)$ in $(1.2)$ is a perturbation of a deterministic matrix, in the sense that

$$
A^{\varepsilon}(x, \omega) \equiv A_{\eta}^{\varepsilon}(x, \omega)=A_{0}^{\varepsilon}(x)+\eta A_{1}^{\varepsilon}(x, \omega),
$$

where $\eta \in \mathbb{R}$ is a small deterministic parameter, $A_{0}^{\varepsilon}$ and $A_{1}^{\varepsilon}$ are bounded matrices, and $A_{0}^{\varepsilon}$ is coercive, uniformly in $\varepsilon$. We also assume that the matrix $A_{\eta}^{\varepsilon}$ itself satisfies the coercivity and boundedness assumptions, uniformly in $\eta$ and $\varepsilon$ (we refer to $[6-8]$ and $[15,25]$ for other perturbative settings).

The principle of the proposed approach is to compute the MsFEM basis set of functions with the deterministic part $A_{0}^{\varepsilon}$ of the matrix $A_{\eta}^{\varepsilon}$, and next to perform Monte-Carlo realizations for the macroscale problem (1.2)-(2.12), where we keep the exact matrix $A_{\eta}^{\varepsilon}$ (and not only its deterministic part). Following the approach sketched in Section 2.1, we first solve (2.7) with $A^{\varepsilon}(x) \equiv A_{0}^{\varepsilon}(x)$, and build the deterministic finite dimensional space

$$
\mathcal{W}_{h}:=\operatorname{span}\left(\phi_{i}^{\varepsilon}, i=1, \ldots, L\right)
$$


following $(2.8)-(2.9)$. We next proceed with a standard Galerkin approximation of (1.2)-(2.12) using $\mathcal{W}_{h}$. For each $m \in\{1, \ldots, M\}$, we consider a realization $A_{\eta}^{\varepsilon, m}(\cdot, \omega)$ and compute $u_{S}^{m}(\cdot, \omega) \in \mathcal{W}_{h}$ such that

$$
\forall v \in \mathcal{W}_{h}, \quad \sum_{\mathbf{K}} \int_{\mathbf{K}}(\nabla v(x))^{T} A_{\eta}^{\varepsilon, m}(x, \omega) \nabla u_{S}^{m}(x, \omega) \mathrm{d} x=\int_{\mathcal{D}} f(x) v(x) \mathrm{d} x .
$$

Since the MsFEM basis functions are only computed once (rather than for each realization of $A_{\eta}^{\varepsilon}(x, \omega)$ ), a large computational gain is expected, and obtained, in comparison to the direct approach described above.

\section{Numerical Simulations}

This section is devoted to the many numerical simulations we have performed. We first discuss some implementation details. Next, we numerically estimate the performance of our approach on various test cases, and assess its sensitivity with respect to the magnitude of $\eta$. We consider in Section 3.2 a test case in dimension one. In Section 3.3, we next study two test cases in dimension two. We also study how the presence in $A_{1}^{\varepsilon}$ (the random component of the matrix $A_{\eta}^{\varepsilon}$ ) of high frequencies that are not present in the deterministic component $A_{0}^{\varepsilon}$, and that are thus not encoded in the highly oscillatory basis functions, affects the accuracy of our approach. In Section 3.4, we eventually compare our approach with a fully deterministic approach.

Let $u_{\eta}^{\varepsilon}$ be the reference solution to $(1.2)-(1.3)$ obtained using a finite element method with a mesh size adapted to the small scale $\varepsilon, u_{S}$ be the approximation given by our approach (described in Sect. 2.2) and $u_{M}$ be the approximation given by the direct approach (in which the MsFEM basis set is recomputed for each realization $A_{\eta}^{\varepsilon, m}(x, \omega)$, as explained at the end of Sect. 2.1). Our goal is to compare the error $u_{S}-u_{\eta}^{\varepsilon}$ of our numerical approximation with the error $u_{M}-u_{\eta}^{\varepsilon}$ of the direct and expensive approach. When $\eta$ is small, we expect the approximation $u_{S}$ to be essentially as accurate as the approximation $u_{M}$, and we show below that this is indeed the case.

In the sequel, we assess the accuracy using the estimators

$$
e_{L^{2}}\left(u_{1}, u_{2}\right)=\mathbb{E}\left(\frac{\left\|u_{1}-u_{2}\right\|_{L^{2}(\mathcal{D})}}{\left\|u_{2}\right\|_{L^{2}(\mathcal{D})}}\right) \quad \text { and } \quad e_{H^{1}}\left(u_{1}, u_{2}\right)=\mathbb{E}\left(\frac{\left\|u_{1}-u_{2}\right\|_{H_{h}^{1}}}{\left\|u_{2}\right\|_{H_{h}^{1}}}\right),
$$

where $u_{1}$ and $u_{2}$ are the solutions obtained with any two different methods, and

$$
\|u\|_{H_{h}^{1}}:=\left(\sum_{\mathbf{K} \in \mathcal{T}_{h}}\|u\|_{H^{1}(\mathbf{K})}^{2}\right)^{1 / 2}
$$

is the broken $H^{1}$ norm. The expectation is in turn computed using a Monte-Carlo method. Considering $M$ realizations $\left\{X_{m}(\omega)\right\}_{1 \leq m \leq M}$ of a random variable, e.g. $X(\omega)=\frac{\left\|u_{1}(\cdot, \omega)-u_{2}(\cdot, \omega)\right\|_{H_{h}^{1}}}{\left\|u_{2}(\cdot, \omega)\right\|_{H_{h}^{1}}}$, we compute the empirical mean $\mu_{M}$ and the empirical standard deviation $\sigma_{M}$ as

$$
\mu_{M}(X)=\frac{1}{M} \sum_{m=1}^{M} X_{m}(\omega), \quad \sigma_{M}^{2}(X)=\frac{1}{M-1} \sum_{m=1}^{M}\left(X_{m}(\omega)-\mu_{M}(X)\right)^{2} .
$$

As a classical consequence of the Central Limit Theorem, the following estimate is commonly employed:

$$
\left|\mathbb{E}(X)-\mu_{M}(X)\right| \leq 1.96 \frac{\sigma_{M}(X)}{\sqrt{M}} .
$$

It provides a practical evaluation of $\mathbb{E}(X)$ from the knowledge of $\mu_{M}(X)$ and $\sigma_{M}(X)$. The numerical parameters have been determined by an empirical study of convergence. For instance, for the reference solution, we choose 
the mesh size $h$ such that the quantity $\frac{\left\|u_{\eta}^{\varepsilon, h}-u_{\eta}^{\varepsilon, h / 2}\right\|_{H^{1}(\mathcal{D})}}{\left\|u_{\eta}^{\varepsilon, h / 2}\right\|_{H^{1}(\mathcal{D})}}$ is smaller than $0.03 \%$, thereby formally admitting that the approximation has converged in $h$. The MsFEM parameters are determined likewise.

All the computations have been performed using FreeFem ++ [33], with the MPI tools.

\subsection{Implementation details}

In the deterministic version of the MsFEM, the same matrix $A^{\varepsilon}$ appears in the definition (2.7) of the basis functions and in the macroscale variational formulation (2.11). This can be used to expedite the computation of the stiffness matrix associated with (2.11). In our approach, described in Section 2.2, the matrix that appears in the definition of the basis functions is $A_{0}^{\varepsilon}$, whereas the macroscale variational problem involves $A_{\eta}^{\varepsilon} \equiv A_{0}^{\varepsilon}+\eta A_{1}^{\varepsilon}$. An additional numerical computation is thus needed.

To solve (2.13), we need to compute, for each element $\mathbf{K}$ and each realization $A_{\eta}^{\varepsilon, \bar{m}}(x, \omega)$, the integrals

$$
\mathcal{K}_{i j}^{\eta, \bar{m}}(\omega)=\int_{\mathbf{K}}\left(\nabla \phi_{i}^{\varepsilon, \mathbf{K}}(x)\right)^{T} A_{\eta}^{\varepsilon, \bar{m}}(x, \omega) \nabla \phi_{j}^{\varepsilon, \mathbf{K}}(x) \mathrm{d} x
$$

where $\phi_{i}^{\varepsilon, \mathbf{K}}$ are deterministic functions. We recall that $A_{\eta}^{\varepsilon}(x, \omega)=A_{0}^{\varepsilon}(x)+\eta A_{1}^{\varepsilon}(x, \omega)$ (see (2.12)). To allow for an efficient evaluation of (3.4), we assume henceforth that $A_{1}^{\varepsilon}$ is of the form

$$
A_{1}^{\varepsilon}(x, \omega)=\sum_{k \in \mathbb{Z}^{d}} \mathbf{1}_{Q+k}\left(\frac{x}{\varepsilon}\right) X_{k}(\omega) B_{\varepsilon}^{k}(x),
$$

where $Q=(0,1)^{d}$, where $\left(X_{k}\right)_{k \in \mathbb{Z}^{d}}$ are scalar random variables, and for any $k \in \mathbb{Z}^{d}, x \mapsto B_{\varepsilon}^{k}(x) \in \mathbb{R}^{d \times d}$ are some deterministic functions. We comment on this assumption in Remark 3.1 below. The important consequence of (3.5) is that we can write the integral (3.4) as a linear combination of deterministic integrals over cells of size $\varepsilon$, with random coefficients. To simplify the notation, we assume that the spatial dimension is $d=2$. We define

$$
p=\left\lfloor\min \left(\frac{y_{i}}{\varepsilon}, \frac{y_{j}}{\varepsilon}, \frac{y_{k}}{\varepsilon}\right)\right\rfloor, \quad q=\left\lfloor\max \left(\frac{y_{i}}{\varepsilon}, \frac{y_{j}}{\varepsilon}, \frac{y_{k}}{\varepsilon}\right)\right\rfloor+1,
$$

where $y_{i}, y_{j}$ and $y_{k}$ are the y-axis coordinates of the three vertex of $\mathbf{K}$ (see Fig. 2). We likewise define the integers $l$ and $m$ (see Fig. 2). We can then write (3.4) as

$$
\mathcal{K}_{i j}^{\eta, \bar{m}}(\omega)=\int_{\mathbf{K}}\left(\nabla \phi_{i}^{\varepsilon, \mathbf{K}}(x)\right)^{T} A_{\eta}^{\varepsilon, \bar{m}}(x, \omega) \nabla \phi_{j}^{\varepsilon, \mathbf{K}}(x) \mathrm{d} x=\mathcal{K}_{i j}^{0, \mathbf{K}}+\eta \sum_{\alpha=p}^{q-1} \sum_{\beta=l}^{m-1} X_{\alpha, \beta}^{\bar{m}}(\omega) \mathcal{K}_{\alpha \beta i j}^{1, \mathbf{K}},
$$

where

$$
\begin{aligned}
\mathcal{K}_{i j}^{0, \mathbf{K}} & =\int_{\mathbf{K}}\left(\nabla \phi_{i}^{\varepsilon, \mathbf{K}}(x)\right)^{T} A_{0}^{\varepsilon}(x) \nabla \phi_{j}^{\varepsilon, \mathbf{K}}(x) \mathrm{d} x, \\
\mathcal{K}_{\alpha \beta i j}^{1, \mathbf{K}} & =\int_{\alpha \varepsilon}^{(\alpha+1) \varepsilon} \int_{\beta \varepsilon}^{(\beta+1) \varepsilon} \mathbf{1}_{\mathbf{K}}(x)\left(\nabla \phi_{i}^{\varepsilon, \mathbf{K}}(x)\right)^{T} B_{\varepsilon}^{\alpha, \beta}(x) \nabla \phi_{j}^{\varepsilon, \mathbf{K}}(x) \mathrm{d} x .
\end{aligned}
$$

We thus compute once the deterministic integrals (3.7) and (3.8). Next, for each realization of $A_{\eta}^{\varepsilon}$, we evaluate the stiffness matrix elements $\mathcal{K}_{i j}^{\eta, \bar{m}}(\omega)$ using the right hand side of (3.6). No numerical quadrature is needed. As a consequence of (3.5), most of the work for assembling the stiffness matrix is only performed once, independently of the number of Monte Carlo realizations. This significantly contributes to the gain in term of computational cost. 


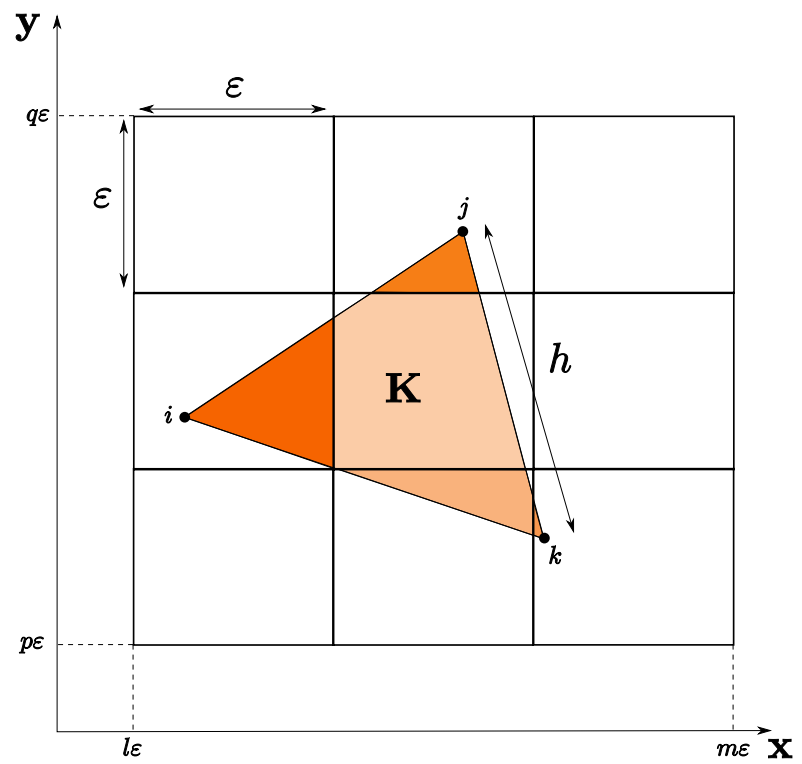

Figure 2. To practically compute the integral (3.4), we write that each element $\mathbf{K}$ (here in dimension $d=2$ ) is a subset of a quadrangle (here $[l \varepsilon, m \varepsilon] \times[p \varepsilon, q \varepsilon]$ ) composed of cells of size $\varepsilon^{d}$.

Remark 3.1. Assumption (3.5) is quite general, and already covers many interesting cases in practice. As explained above, the point in (3.5) is that $A_{1}^{\varepsilon}$ is a direct product (or here, a sum of direct products) of a function depending on $x$ with a random variable that only depends on $\omega$. Otherwise stated, $A_{1}^{\varepsilon}(x, \omega)$ depends linearly, in an explicit way, of $\omega$. A similar assumption is made when applying reduced basis methods [45] to a problem of the type

Find $u_{\lambda}$ such that, for any $v, a\left(u_{\lambda}, v ; \lambda\right)=b(v)$,

where $a(\cdot, \cdot ; \lambda)$ is a bilinear form parameterized by $\lambda$. Assume this problem has been solved for some values $\left\{\lambda_{i}\right\}_{i=1}^{I}$ of the parameter, yielding the functions $\left\{u_{\lambda_{i}}\right\}_{i=1}^{I}$. Under the assumption that $a(\cdot, \cdot ; \lambda)=a_{0}(\cdot, \cdot)+\lambda a_{1}(\cdot, \cdot)$ (namely, $a(\cdot, \cdot ; \lambda)$ depends linearly on $\lambda$ ), one can precompute the stiffness matrix elements $a_{0}\left(u_{\lambda_{i}}, u_{\lambda_{j}}\right)$ and $a_{1}\left(u_{\lambda_{i}}, u_{\lambda_{j}}\right)$ for any $1 \leq i, j \leq I$. This allows to next perform a very efficient Galerkin approximation of the problem (3.9) (for any $\lambda$ ) on the space $\operatorname{Span}\left(u_{\lambda_{i}}, i=1, \ldots, I\right)$.

\subsection{One-dimensional test-case}

The purpose of this section is threefold. We first calibrate the number $M$ of realizations considered for the Monte-Carlo method for the two-dimensional numerical experiments that we consider in the sequel. We next investigate how the accuracy of our approach depends on $\eta$ and on the presence of frequencies in the random coefficient $a_{\eta}^{\varepsilon}$ that are not taken into account in the MsFEM basis set functions. The low computational costs that we face in this one-dimensional situation allow us to test our approach more comprehensively than in the two-dimensional test-cases described below.

Let $\left(X_{k}\right)_{k \in \mathbb{Z}}$ denote a sequence of independent, identically distributed scalar random variables uniformly distributed in $[0,1]$. We consider the random coefficient

$$
a_{\eta}^{\varepsilon}(x, \omega)=\sum_{k \in \mathbb{Z}} \mathbf{1}_{(k, k+1]}\left(\frac{x}{\varepsilon}\right)\left(5+50 \sin ^{2}\left(\frac{\pi x}{\varepsilon}\right)+\eta X_{k}(\omega) \kappa \sin ^{2}\left(\frac{\zeta \pi x}{\varepsilon}\right)\right),
$$




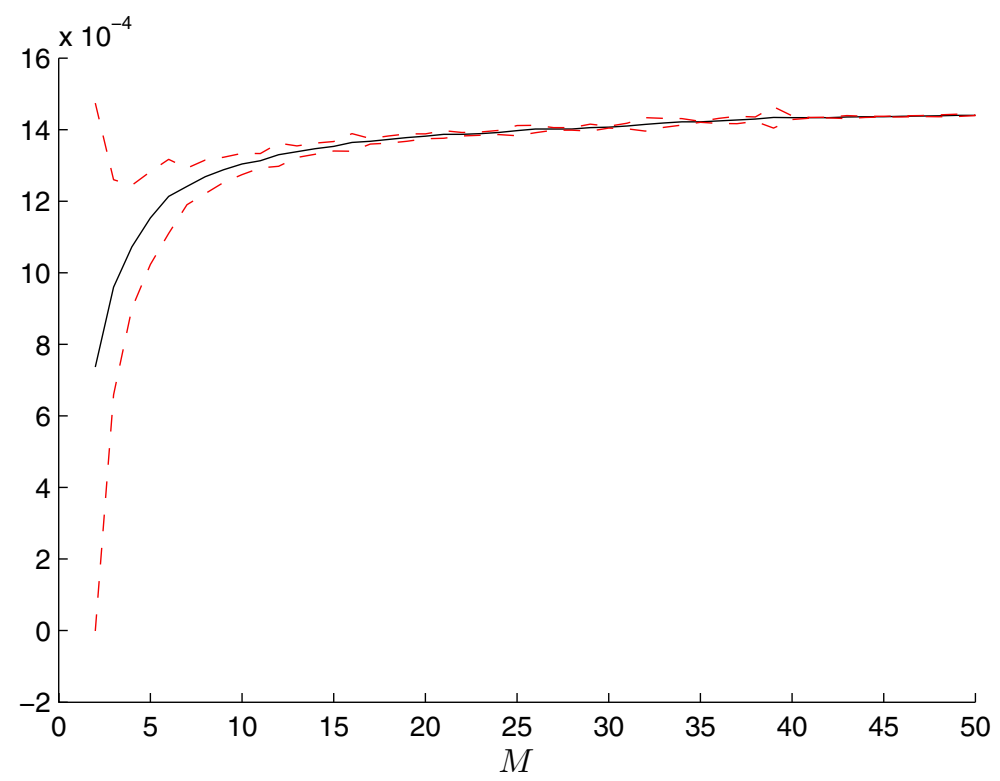

Figure 3. Convergence of the indicator $e_{H^{1}}\left(u_{M}, u_{\eta}^{\varepsilon}\right.$ ) (see (3.1)), for $\eta=1, \zeta=1$ and $\kappa=55$. For each value of $M$, we plot the empirical mean along with its confidence interval, computed from the first $M$ realizations. We only plot the results for the first 50 realizations.

which is a particular example of the expansion (2.12) with

$$
a_{0}^{\varepsilon}(x)=5+50 \sin ^{2}\left(\frac{\pi x}{\varepsilon}\right) \quad \text { and } \quad a_{1}^{\varepsilon}(x, \omega)=\sum_{k \in \mathbb{Z}} \mathbf{1}_{(k, k+1]}\left(\frac{x}{\varepsilon}\right) X_{k}(\omega) \kappa \sin ^{2}\left(\frac{\zeta \pi x}{\varepsilon}\right),
$$

and that satisfies the structural assumption (3.5). We set $\varepsilon=0.025$ and choose $\kappa$ such that the quantity

$$
\mathcal{R}(\kappa, \zeta)=\left\|\frac{a_{1}^{\varepsilon}}{a_{0}^{\varepsilon}}\right\|_{L^{\infty}(\mathcal{D} \times \Omega)}=\operatorname{SupEss}_{\omega \in \Omega}\left\|\frac{a_{1}^{\varepsilon}(\cdot, \omega)}{a_{0}^{\varepsilon}}\right\|_{L^{\infty}(\mathcal{D})}
$$

has the same value $\mathcal{R}(\kappa, \zeta)=1$ for the three different values of $\zeta=\{1,3,7\}$ we consider below. This yields the choices $(\kappa, \zeta)=(55,1),(\kappa, \zeta)=(14.38,3)$ and $(\kappa, \zeta)=(8.39,7)$. We analytically compute the reference function $u_{\eta}^{\varepsilon}$, solution to

$$
-\frac{\mathrm{d}}{\mathrm{d} x}\left(a_{\eta}^{\varepsilon}(x, \omega) \frac{d u_{\eta}^{\varepsilon}}{\mathrm{d} x}(x, \omega)\right)=1 \text { in }(0,1), \quad u_{\eta}^{\varepsilon}(0, \omega)=u_{\eta}^{\varepsilon}(1, \omega)=0,
$$

as well as the MsFEM basis functions for both approaches. Let $u_{M}$ and $u_{S}$ be the approximation of $u_{\eta}^{\varepsilon}$ by the two MsFEM approaches described above, where the coarse mesh size is $h=1 / 30$.

We first calibrate the number of independent realizations to accurately approximate the exact expectation in (3.1) by the empirical mean (3.3). To this aim, we present on Figure 3 the mean and the confidence interval computed using (3.3) for an increasing number $M$ of realizations (we compute up to 1000 independent realizations). We check that this indicator reaches a plateau for $M \geq 30$, and thus converges fast. On this example, considering 30 realizations is hence sufficient to accurately compute the error (3.1). Based on this observation, we will only consider $M=30$ realizations in the two dimensional examples of Section 3.3.

Remark 3.2. There is no reason to think that the calibration of our parameters that we perform in the onedimensional situation provides an adequate adaptation of these parameters for the higher dimensional setting. 
TABLE $1 . H^{1}(0,1)$ error $(3.1)$ (in $\%$ ) for $\kappa=55$ and $\zeta=1$.

\begin{tabular}{lccc}
\hline$\eta$ & $e_{H^{1}}\left(u_{M}, u_{\eta}^{\varepsilon}\right)$ & $e_{H^{1}}\left(u_{S}, u_{\eta}^{\varepsilon}\right)$ & $e_{H^{1}}\left(u_{S}, u_{M}\right)$ \\
\hline 1 & $0.14644 \pm 0.00036$ & $2.62550 \pm 0.02696$ & $2.44359 \pm 0.02696$ \\
0.1 & $0.16001 \pm 0.00006$ & $0.15021 \pm 0.00051$ & $0.07036 \pm 0.00044$ \\
0.01 & $0.16258 \pm 0.00000$ & $0.10837 \pm 0.00002$ & $0.04825 \pm 0.00025$ \\
\hline
\end{tabular}

TABLe 2. $H^{1}(0,1)$ error $(3.1)$ (in $\%$ ) for $\kappa=14.38$ and $\zeta=3$

\begin{tabular}{lccc}
\hline$\eta$ & $e_{H^{1}}\left(u_{M}, u_{\eta}^{\varepsilon}\right)$ & $e_{H^{1}}\left(u_{S}, u_{\eta}^{\varepsilon}\right)$ & $e_{H^{1}}\left(u_{S}, u_{M}\right)$ \\
\hline 1 & $0.18269 \pm 0.00030$ & $2.38950 \pm 0.02277$ & $2.23869 \pm 0.02230$ \\
0.1 & $0.16529 \pm 0.00003$ & $0.14959 \pm 0.00055$ & $0.08082 \pm 0.00041$ \\
0.01 & $0.16314 \pm 0.00000$ & $0.10840 \pm 0.00000$ & $0.04954 \pm 0.00001$ \\
\hline
\end{tabular}

TABLE 3. $H^{1}(0,1)$ error $(3.1)$ (in $\%$ ) for $\kappa=8.39$ and $\zeta=7$.

\begin{tabular}{lccc}
\hline$\eta$ & $e_{H^{1}}\left(u_{M}, u_{\eta}^{\varepsilon}\right)$ & $e_{H^{1}}\left(u_{S}, u_{\eta}^{\varepsilon}\right)$ & $e_{H^{1}}\left(u_{S}, u_{M}\right)$ \\
\hline 1 & $0.17436 \pm 0.00026$ & $2.34495 \pm 0.02105$ & $2,27358 \pm 0.02089$ \\
0.1 & $0.16465 \pm 0.00004$ & $0.15748 \pm 0.00067$ & $0.09803 \pm 0.00053$ \\
0.01 & $0.16308 \pm 0.00000$ & $0.10846 \pm 0.00000$ & $0.05054 \pm 0.00001$ \\
\hline
\end{tabular}

We however see no other manner to proceed and the approach has indeed provided us with good results (see Rem. 3.4 below).

Note also that the MsFEM approach is much more accurate in the one-dimensional setting than in the twodimensional setting (compare Tabs. 1, 2 and 3 with Tabs. 9 and 10 below). This is due to the specificity of the one dimensional setting. However, one-dimensional examples remain relevant for e.g. assessing how the MsFEM accuracy depends on $\eta$.

Note finally that the fact that considering $M=30$ independent realizations is sufficient is related to our specific context, namely stochastic homogenization. In other application fields of Monte Carlo methods, larger values of $M$ are often required.

We now check how the accuracy of our approach depends on $\eta$. In Tables 1, 2, 3, 4, 5 and 6 , we report the estimators (3.1), along with their confidence intervals, for various choices of $(\kappa, \zeta)$ that all correspond to $\mathcal{R}(\kappa, \zeta)=1$. For $\eta \leq 0.1$, we observe that $\left\|u_{S}-u_{\eta}^{\varepsilon}\right\|$ and $\left\|u_{M}-u_{\eta}^{\varepsilon}\right\|$ are of the same order of magnitude, and are both larger than $\left\|u_{M}-u_{S}\right\|$ (both in $L^{2}$ and broken $H^{1}$ norms). We thus obtain the same accuracy with the direct and the weak stochastic MsFEM approaches, whereas the weak stochastic MsFEM is computationally (much) less expensive. For $\eta=1$, as expected, the accuracy of the approximation $u_{S}$ deteriorates. The accuracy of $u_{M}$ is independent of $\eta$.

Remark 3.3. In Section 4, we estimate in the $H^{1}$ (broken) norm the error between the reference solution $u_{\eta}^{\varepsilon}$ and the weak stochastic MsFEM solution $u_{S}$. For information, we also include in Tables $1-6$ the numerical comparison in the $L^{2}$ norm.

We now turn to a different question. In the example considered here, some frequencies present in $a_{1}^{\varepsilon}$ do not appear in $a_{0}^{\varepsilon}$, and are thus not captured in the highly oscillatory basis functions $\phi_{i}^{\varepsilon}$. We now show that our approach can still handle this case, provided the amplitude of these modes remains small.

We first consider the case when the amplitude $\kappa$ associated to the frequency $\zeta$ is kept constant, and compare the performance of our approach in the case $\zeta=1$ and $\zeta=3$. In the latter case, a relevant high frequency is not taken into account in the basis set functions. Comparing Tables 1 and 4 (corresponding to $\zeta=1$ ) with Tables 7 and 8 (corresponding to $\zeta=3$ ) for a given value of $\eta$, we see that the accuracy of our approach deteriorates. This is not unexpected, of course. Otherwise stated, to achieve a given accuracy (say an error of $0.15 \%$ in the 
TABLE 4. $L^{2}(0,1)$ error $(3.1)$ (in $\%$ ) for $\kappa=55$ and $\zeta=1$.

\begin{tabular}{lccc}
\hline$\eta$ & $e_{L^{2}}\left(u_{M}, u_{\eta}^{\varepsilon}\right)$ & $e_{L^{2}}\left(u_{S}, u_{\eta}^{\varepsilon}\right)$ & $e_{L^{2}}\left(u_{S}, u_{M}\right)$ \\
\hline 1 & $0.00018 \pm 0.00000$ & $0.07286 \pm 0.00317$ & $0.06861 \pm 0.00306$ \\
0.1 & $0.00018 \pm 0.00000$ & $0.00045 \pm 0.00002$ & $0.00024 \pm 0.00001$ \\
0.01 & $0.00018 \pm 0.00000$ & $0.00015 \pm 0.00000$ & $0.00002 \pm 0.00000$ \\
\hline
\end{tabular}

TABLe 5. $L^{2}(0,1)$ error (3.1) (in $\%$ ) for $\kappa=14.38$ and $\zeta=3$.

\begin{tabular}{lccc}
\hline$\eta$ & $e_{L^{2}}\left(u_{M}, u_{\eta}^{\varepsilon}\right)$ & $e_{L^{2}}\left(u_{S}, u_{\eta}^{\varepsilon}\right)$ & $e_{L^{2}}\left(u_{S}, u_{M}\right)$ \\
\hline 1 & $0.00019 \pm 0.00000$ & $0.06658 \pm 0.00270$ & $0.06238 \pm 0.00261$ \\
0.1 & $0.00018 \pm 0.00000$ & $0.00036 \pm 0.00001$ & $0.00019 \pm 0.00001$ \\
0.01 & $0.00018 \pm 0.00000$ & $0.00015 \pm 0.00000$ & $0.00002 \pm 0.00000$ \\
\hline
\end{tabular}

TABLE 6. $L^{2}(0,1)$ error $(3.1)$ (in $\%$ ) for $\kappa=8.39$ and $\zeta=7$.

\begin{tabular}{lccc}
\hline$\eta$ & $e_{L^{2}}\left(u_{M}, u_{\eta}^{\varepsilon}\right)$ & $e_{L^{2}}\left(u_{S}, u_{\eta}^{\varepsilon}\right)$ & $e_{L^{2}}\left(u_{S}, u_{M}\right)$ \\
\hline 1 & $0.00018 \pm 0.00000$ & $0.08903 \pm 0.00310$ & $0.08410 \pm 0.00261$ \\
0.1 & $0.00018 \pm 0.00000$ & $0.00037 \pm 0.00002$ & $0.00016 \pm 0.00000$ \\
0.01 & $0.00018 \pm 0.00000$ & $0.00015 \pm 0.00000$ & $0.00003 \pm 0.00000$ \\
\hline
\end{tabular}

TABle 7. $H^{1}(0,1)$ error (3.1) (in $\left.\%\right)$ for $\kappa=55$ and $\zeta=3$.

\begin{tabular}{lccc}
\hline$\eta$ & $e_{H^{1}}\left(u_{M}, u_{\eta}^{\varepsilon}\right)$ & $e_{H^{1}}\left(u_{S}, u_{\eta}^{\varepsilon}\right)$ & $e_{H^{1}}\left(u_{S}, u_{M}\right)$ \\
\hline 1 & $0.21826 \pm 0.00073$ & $12.30047 \pm 0.10647$ & $12.01694 \pm 0.10617$ \\
0.1 & $0.17142 \pm 0.00013$ & $0.59293 \pm 0.00519$ & $0.49523 \pm 0.00489$ \\
0.01 & $0.16383 \pm 0.00001$ & $0.11448 \pm 0.00014$ & $0.05247 \pm 0.00007$ \\
\hline
\end{tabular}

TABLE 8. $L^{2}(0,1)$ error (3.1) (in \%) for $\kappa=55$ and $\zeta=3$.

\begin{tabular}{lccc}
\hline$\eta$ & $e_{L^{2}}\left(u_{M}, u_{\eta}^{\varepsilon}\right)$ & $e_{L^{2}}\left(u_{S}, u_{\eta}^{\varepsilon}\right)$ & $e_{L^{2}}\left(u_{S}, u_{M}\right)$ \\
\hline 1 & $0.00022 \pm 0.00000$ & $1.53780 \pm 0.03878$ & $1.51837 \pm 0.00385$ \\
0.1 & $0.00019 \pm 0.00000$ & $0.00503 \pm 0.00027$ & $0.00406 \pm 0.00024$ \\
0.01 & $0.00018 \pm 0.00000$ & $0.00018 \pm 0.00000$ & $0.00005 \pm 0.00000$ \\
\hline
\end{tabular}

broken $H^{1}$ norm), we need to take smaller values of $\eta$ (namely $\eta \leq 0.01$ ) when $\zeta=3$ than when $\zeta=1$ (in which case $\eta=0.1$ is already a sufficiently small value).

We now run the comparison differently. As we increase the gap between the frequency present in $a_{1}^{\varepsilon}$ and that present in $a_{0}^{\varepsilon}$ (i.e., as we increase $\zeta$ ), we simultaneously decrease the amplitude $\kappa$ of that mode. In practice, we do this by keeping constant the parameter $\mathcal{R}(\kappa, \zeta)$ defined by (3.10). Then the accuracy of our approach remains constant, and is independent of $\zeta$. See indeed the numerical results of Tables $1-6$, that all correspond to the choice $\mathcal{R}(\kappa, \zeta)=1$, for three different values of $\zeta$. We observe that, at fixed $\eta$, errors are comparable, and independent of the value of $(\kappa, \zeta)$.

In conclusion, the accuracy of our approach depends both on the amplitude $\kappa$ and the value $\zeta$ of the high frequency not taken into account in the MsFEM basis set functions. If $\zeta$ and $\kappa$ are scaled so that $\mathcal{R}(\kappa, \zeta)$ remains constant (which implies that $\kappa$ decreases if $\zeta$ increases), then the accuracy of our approach remains constant.

\subsection{Two-dimensional test-cases}

We now test our approach on two-dimensional test cases. Using the first test case, we show, similarly to the one-dimensional situation, that the weak stochastic MsFEM yields accurate results, provided the parameter $\eta$ is sufficiently small, and provided that the amplitude associated to frequencies present in $A_{\eta}^{\varepsilon}$ but not encoded 
TABLE $9 . H^{1}(\mathcal{D})$ error (3.1) (in \%) for $\kappa=73.61$ and $\zeta=1$.

\begin{tabular}{lccc}
\hline$\eta$ & $e_{H^{1}}\left(u_{M}, u_{\eta}^{\varepsilon}\right)$ & $e_{H^{1}}\left(u_{S}, u_{\eta}^{\varepsilon}\right)$ & $e_{H^{1}}\left(u_{S}, u_{M}\right)$ \\
\hline 1 & $7.8437 \pm 0.1350$ & $19.8818 \pm 0.4123$ & $18.8662 \pm 0.4216$ \\
0.1 & $6.8053 \pm 0.0165$ & $7.3868 \pm 0.0276$ & $3.1528 \pm 0.0517$ \\
0.01 & $6.7338 \pm 0.0017$ & $6.9795 \pm 0.0016$ & $1.8763 \pm 0.0013$ \\
\hline
\end{tabular}

TABLE $10 . H^{1}(\mathcal{D})$ error $(3.1)$ (in $\%$ ) for $\kappa=10$ and $\zeta=3$.

\begin{tabular}{lccc}
\hline$\eta$ & $e_{H^{1}}\left(u_{M}, u_{\eta}^{\varepsilon}\right)$ & $e_{H^{1}}\left(u_{S}, u_{\eta}^{\varepsilon}\right)$ & $e_{H^{1}}\left(u_{S}, u_{M}\right)$ \\
\hline 1 & $6.7224 \pm 0.0368$ & $12.7292 \pm 0.2172$ & $10.8128 \pm 0.2442$ \\
0.1 & $6.7154 \pm 0.0044$ & $7.1069 \pm 0.0128$ & $2.2925 \pm 0.0206$ \\
0.01 & $6.1725 \pm 0.0004$ & $6.9770 \pm 0.0010$ & $1.8504 \pm 0.0003$ \\
\hline
\end{tabular}

in the deterministic basis functions is small (see Sect. 3.3.1). Next, in Section 3.3.2, we consider a test case similar to a classical benchmark test case of the literature. We again observe that our approach is efficient. For both cases, we show that the parameter $\eta$ does not need to be extremely small for our approach to be highly competitive.

\subsubsection{A multi-frequency case}

In line with what we observed in the one-dimensional case, we show here that the weak stochastic MsFEM provides interesting results even in the case when not all the frequencies present in $A_{\eta}^{\varepsilon}$ are captured in the deterministic basis functions, provided their amplitude is not too large. To this aim, we consider the following numerical example.

Let $\left(X_{k, l}\right)_{(k, l) \in \mathbb{Z}^{2}}$ denote a sequence of independent, identically distributed scalar random variables uniformly distributed in the interval $[0,1]$. We consider the random matrix

$$
A_{\eta}^{\varepsilon}(x, y, \omega)=a_{0}^{\varepsilon}(x, y) \operatorname{Id}_{2}+\eta a_{1}^{\varepsilon}(x, y, \omega) \operatorname{Id}_{2},
$$

with

$$
\begin{aligned}
a_{0}^{\varepsilon}(x, y) & =5+50 \sin ^{2}\left(\frac{\pi x}{\varepsilon}\right) \sin ^{2}\left(\frac{\pi y}{\varepsilon}\right), \\
a_{1}^{\varepsilon}(x, y, \omega) & =\sum_{(k, l) \in \mathbb{Z}^{2}} \mathbf{1}_{(k, k+1]}\left(\frac{x}{\varepsilon}\right) \mathbf{1}_{(l, l+1]}\left(\frac{y}{\varepsilon}\right)\left(X_{k, l}(\omega) \kappa \sin ^{2}\left(\frac{\zeta \pi x}{\varepsilon}\right) \sin ^{2}\left(\frac{\zeta \pi y}{\varepsilon}\right)\right) .
\end{aligned}
$$

Again, this choice is a particular example of the expansion (2.12) satisfying the structural assumption (3.5). We consider two different values of $\zeta$, namely $\zeta=1$ and $\zeta=3$. As in the previous test case, the frequency $\zeta$ is not present in the deterministic part of $A_{\eta}^{\varepsilon}$, and thus not encoded in the basis functions. In line with what we observed in Section 3.2, we choose the amplitude $\kappa$ associated to that frequency such that the quantity (3.10) has the same value $\mathcal{R}(\kappa, \zeta)=1$ for both values of $\zeta$, which yields the choices $(\kappa, \zeta)=(73.61,1)$ and $(\kappa, \zeta)=(10,3)$. We compute $u_{\eta}^{\varepsilon}$ solution to

$$
-\operatorname{div}\left[A_{\eta}^{\varepsilon}(\cdot, \omega) \nabla u_{\eta}^{\varepsilon}(\cdot, \omega)\right]=1 \text { in } \mathcal{D}, \quad u_{\eta}^{\varepsilon}(\cdot, \omega)=0 \quad \text { on } \partial \mathcal{D},
$$

on the domain $\mathcal{D}=(0,1)^{2}$ with $\varepsilon=0.025$. Let $u_{M}$ and $u_{S}$ be its approximation by the two MsFEM approaches described above. The numerical parameters for the computation are again determined using an empirical study of convergence. We use for the reference solution $u_{\eta}^{\varepsilon}$ a fine mesh of size $h_{f}=\varepsilon / 40$. The MsFEM basis functions are computed in each element $\mathbf{K}$ using a mesh of size $h_{M}=\varepsilon / 80$. The oversampling parameter (i.e. the scale ratio of the homothetic transformation between $\mathbf{K}$ and $\mathbf{S}$, see Fig. 1) is equal to 3 . The coarse mesh size is 
TABLE 11. $L^{2}(\mathcal{D})$ error $(3.1)$ (in $\%$ ) for $\kappa=73.61$ and $\zeta=1$.

\begin{tabular}{lccc}
\hline$\eta$ & $e_{L^{2}}\left(u_{M}, u_{\eta}^{\varepsilon}\right)$ & $e_{L^{2}}\left(u_{S}, u_{\eta}^{\varepsilon}\right)$ & $e_{L^{2}}\left(u_{S}, u_{M}\right)$ \\
\hline 1 & $1.4355 \pm 0.0795$ & $4.1649 \pm 0.1652$ & $2.8468 \pm 0.1694$ \\
0.1 & $1.0630 \pm 0.0108$ & $1.1369 \pm 0.0075$ & $0.1441 \pm 0.0354$ \\
0.01 & $1.0211 \pm 0.0011$ & $1.1512 \pm 0.0007$ & $0.1351 \pm 0.0014$ \\
\hline
\end{tabular}

TABLE $12 . L^{2}(\mathcal{D})$ error $(3.1)$ (in $\%$ ) for $\kappa=10$ and $\zeta=3$.

\begin{tabular}{lccc}
\hline$\eta$ & $e_{L^{2}}\left(u_{M}, u_{\eta}^{\varepsilon}\right)$ & $e_{L^{2}}\left(u_{S}, u_{\eta}^{\varepsilon}\right)$ & $e_{L^{2}}\left(u_{S}, u_{M}\right)$ \\
\hline 1 & $1.0744 \pm 0.0127$ & $1.8433 \pm 0.0582$ & $0.8426 \pm 0.0832$ \\
0.1 & $1.0226 \pm 0.0015$ & $1.1249 \pm 0.0038$ & $0.1147 \pm 0.0073$ \\
0.01 & $1.0170 \pm 0.0001$ & $1.1551 \pm 0.0004$ & $0.1427 \pm 0.0003$ \\
\hline
\end{tabular}

$h=1 / 30$. In view of the results of Section 3.2, we consider $M=30$ independent realizations, which will prove to again be sufficient to obtain accurate results.

In Tables 9 and 10 (Tabs. 11 and 12 respectively), we report the estimator (3.1), along with its confidence interval, for the broken $H^{1}(\mathcal{D})$ norm and for the $L^{2}(\mathcal{D})$ norm, respectively. The results obtained here confirm our observations in the one-dimensional setting (Sect. 3.2):

- for given $\zeta$ and $\kappa$, we observe that, when $\eta$ is sufficiently small (here, $\eta \leq 0.1$ ), the alternative approach provides a solution $u_{S}$ that is an approximation of $u_{\eta}^{\varepsilon}$ as accurate as $u_{M}$, for a much smaller computational cost (as the MsFEM basis set has only been computed once rather than for each independent realization of $\left.A_{\eta}^{\varepsilon}\right)$.

- our approach yields accurate results even if the frequency $\zeta$ is not encoded in the basis functions $\phi_{i}^{\varepsilon}$, provided the associated amplitude $\kappa$ is scaled accordingly. Figures in Table 9 (respectively Tab. 11) are very close to those of Table 10 (respectively Tab. 12). This confirms that the error made by the weak stochastic MsFEM seems to be independent of $\kappa$ and $\zeta$, provided these two parameters are scaled so that $\mathcal{R}(\kappa, \zeta)$ remains constant. If $\zeta$ becomes different than 1 , the frequency present in $a_{0}^{\varepsilon}$, then the amplitude $\kappa$ associated to the frequency $\zeta$ has to decrease to keep $\mathcal{R}(\kappa, \zeta)$ (and thus the accuracy of $u_{S}$ ) constant.

These observations again demonstrate the efficiency of the approach.

Remark 3.4. In Tables 9-12, we observe that the size of the confidence interval is much smaller than the distance between two different errors. This a posteriori validates the choice of the number $M$ of Monte Carlo realizations according to the calibration we performed in the one-dimensional setting. In the two-dimensional setting studied here, we observe that considering $M=30$ realizations is again sufficient. The same conclusion holds for results presented in Tables 13-16 below.

\subsubsection{A classical test case}

We consider in this section a test case similar to a classical test case of the literature (see e.g. $[19,32,37,39]$ ). Let $\left(X_{k, l}\right)_{(k, l) \in \mathbb{Z}^{2}}$ denote a sequence of independent, identically distributed scalar random variables uniformly distributed in the interval $[0,1]$. We consider the random matrix

$$
A_{\eta}^{\varepsilon}(x, y, \omega)=\sum_{(k, l) \in \mathbb{Z}^{2}} \mathbf{1}_{(k, k+1]}\left(\frac{x}{\varepsilon}\right) \mathbf{1}_{(l, l+1]}\left(\frac{y}{\varepsilon}\right)\left(\frac{2+P \sin (2 \pi x / \varepsilon)}{2+P \sin (2 \pi y / \varepsilon)}+\frac{2+\sin (2 \pi y / \varepsilon)}{2+P \sin (2 \pi x / \varepsilon)}\right)\left(1+\eta X_{k, l}(\omega)\right) \operatorname{Id}_{2},
$$

with $P=1.8$ and $\varepsilon=0.025$. We compute the reference solution $u_{\eta}^{\varepsilon}$ and its two approximations $u_{M}$ and $u_{S}$ with the same numerical parameters as in Section 3.3.1.

In Tables 13 and 14, we report the estimator (3.1), along with its confidence interval, for the broken $H^{1}(\mathcal{D})$ norm and for the $L^{2}(\mathcal{D})$ norm, respectively. We again see that, when $\eta$ is sufficiently small, $u_{S}$ is an approximation 
TABLE $13 . H^{1}(\mathcal{D})$ error $(3.1)$ (in $\left.\%\right)$.

\begin{tabular}{lccc}
\hline$\eta$ & $e_{H^{1}}\left(u_{M}, u_{\eta}^{\varepsilon}\right)$ & $e_{H^{1}}\left(u_{S}, u_{\eta}^{\varepsilon}\right)$ & $e_{H^{1}}\left(u_{S}, u_{M}\right)$ \\
\hline 1 & $8.1154 \pm 0.1913$ & $17.3678 \pm 0.7784$ & $15.5113 \pm 0.8689$ \\
0.1 & $7.1664 \pm 0.0199$ & $7.0524 \pm 0.0705$ & $2.5638 \pm 0.1006$ \\
0.01 & $7.1453 \pm 0.0020$ & $7.2837 \pm 0.0067$ & $1.3882 \pm 0.0020$ \\
\hline
\end{tabular}

TABLE $14 . L^{2}(\mathcal{D})$ error $(3.1)$ (in \%).

\begin{tabular}{lccc}
\hline$\eta$ & $e_{L^{2}}\left(u_{M}, u_{\eta}^{\varepsilon}\right)$ & $e_{L^{2}}\left(u_{S}, u_{\eta}^{\varepsilon}\right)$ & $e_{L^{2}}\left(u_{S}, u_{M}\right)$ \\
\hline 1 & $0.5620 \pm 0.0803$ & $1.6855 \pm 0.4860$ & $1.4739 \pm 0.5048$ \\
0.1 & $0.5354 \pm 0.0160$ & $0.5688 \pm 0.0630$ & $0.1984 \pm 0.0712$ \\
0.01 & $0.5347 \pm 0.0012$ & $0.6192 \pm 0.0054$ & $0.1072 \pm 0.0032$ \\
\hline
\end{tabular}

TABLE 15. $H^{1}(\mathcal{D})$ error $(3.1)($ in $\%)$.

\begin{tabular}{lc}
\hline$\eta$ & $e_{H^{1}}\left(u_{S}, u_{\eta}^{\varepsilon}\right)$ \\
\hline 1 & $17.3678 \pm 0.7784$ \\
0.5 & $15.9578 \pm 0.3461$ \\
0.25 & $10.6130 \pm 0.1591$ \\
0.1 & $7.0524 \pm 0.0705$ \\
0.01 & $7.2837 \pm 0.0067$ \\
\hline
\end{tabular}

TABLE 16. $L^{2}(\mathcal{D})$ error $(3.1)$ (in $\left.\%\right)$.

\begin{tabular}{lc}
\hline$\eta$ & $e_{L^{2}}\left(u_{S}, u_{\eta}^{\varepsilon}\right)$ \\
\hline 1 & $1.6855 \pm 0.4860$ \\
0.5 & $1.0246 \pm 0.4414$ \\
0.25 & $0.5291 \pm 0.2285$ \\
0.1 & $0.5688 \pm 0.0630$ \\
0.01 & $0.6192 \pm 0.0054$ \\
\hline
\end{tabular}

of the reference solution $u_{\eta}^{\varepsilon}$ as accurate as $u_{M}$. In Tables 15 and 16, we report on the accuracy of $u_{S}$ for more values of $\eta$. Assuming that the accuracy of $u_{M}$ does not depend on $\eta$ (which is consistent with the results reported in Tabs. 13 and 14), we see that our approach is as accurate as the direct, expensive MsFEM approach, as soon as $\eta \leq 0.1$ (if we use the broken $H^{1}$ norm to assess accuracy) and $\eta \leq 0.25$ (if we rather use the $L^{2}$ norm). The parameter $\eta$ hence does not need to be extremely small for our approach to be highly competitive.

\subsection{Comparison to a fully deterministic approach}

Our setting being a perturbation of a deterministic setting,

$$
A^{\varepsilon}(x, \omega) \equiv A_{\eta}^{\varepsilon}(x, \omega)=A_{0}^{\varepsilon}(x)+\eta A_{1}^{\varepsilon}(x, \omega),
$$

it seems a good, simple strategy to consider the following completely deterministic MsFEM approach (compare with our approach described in Sect. 2.2):

- consider the same deterministic finite dimensional space $\mathcal{W}_{h}$ as in our approach, where we solve (2.7) with $A^{\varepsilon}(x) \equiv A_{0}^{\varepsilon}(x)$ 
TABle 17. Estimator (3.12) for the test-case of Section 3.2 (left: $\kappa=8.39$ and $\zeta=7$; right: $\kappa=55$ and $\zeta=1)$.

\begin{tabular}{lccc}
\hline$\eta$ & $\operatorname{Std}\left(u_{\eta}^{\varepsilon}\right)$ & $\operatorname{Std}\left(u_{M}\right)$ & $\operatorname{Std}\left(u_{S}\right)$ \\
\hline 1 & 4.02 & 4.02 & 5.56 \\
0.25 & 1.20 & 1.20 & 1.40 \\
0.1 & 0.505 & 0.505 & 0.559 \\
0.05 & 0.257 & 0.257 & 0.280 \\
0.01 & 0.0522 & 0.0522 & 0.0560 \\
\hline
\end{tabular}

\begin{tabular}{lccc}
\hline$\eta$ & $\operatorname{Std}\left(u_{\eta}^{\varepsilon}\right)$ & $\operatorname{Std}\left(u_{M}\right)$ & $\operatorname{Std}\left(u_{S}\right)$ \\
\hline 1 & 5.05 & 5.06 & 6.50 \\
0.25 & 1.44 & 1.44 & 1.63 \\
0.1 & 0.60 & 0.60 & 0.65 \\
0.05 & 0.30 & 0.30 & 0.33 \\
0.01 & 0.061 & 0.061 & 0.065 \\
\hline
\end{tabular}

- define next $u_{D} \in \mathcal{W}_{h}$ such that

$$
\forall v \in \mathcal{W}_{h}, \quad \sum_{\mathbf{K}} \int_{\mathbf{K}}(\nabla v)^{T} A_{0}^{\varepsilon} \nabla u_{D}=\int_{\mathcal{D}} f v
$$

In contrast to our approach (see (2.13)), the above macroscopic problem only depends on the deterministic part of $A_{\eta}^{\varepsilon}$.

It turns out that, with respect to estimators (3.1), the approximation $u_{D}$ is essentially as accurate as our approximation $u_{S}$ when $\eta$ is small (results not shown). However, the approximation $u_{D}$ being deterministic, all the information about the variance of the reference solution $u_{\eta}^{\varepsilon}(\cdot, \omega)$ cannot be approximated using $u_{D}$. In sharp contrast, our approximation $u_{S}(\cdot, \omega)$ yields accurate approximations for many such quantities of interest, as we now show.

Consider first the one-dimensional test case of Section 3.2, and define, for any $u$,

$$
\operatorname{Std}(u)=\frac{\sqrt{\int_{0}^{1} \operatorname{Var} u(x, \cdot) \mathrm{d} x}}{\mathbb{E}\left[\|u\|_{L^{2}(0,1)}\right]} .
$$

This quantity measures the variance of $u$, integrated over the computational domain $(0,1)$. The results are shown on Table 17 . We see that the results provided by our approximation $u_{S}$ are very close to the reference results (there is less than $10 \%$ of error when $\eta \leq 0.1$ ). The variance of the solution (in the sense of $(3.12)$ ) is therefore well-captured by the approach. This is not the case for the deterministic approach described above, since $\operatorname{Std}\left(u_{D}\right)=0$.

Consider now, for the two-dimensional test-case of Section 3.3.1, the normalized standard error of $\|u(\cdot, \omega)\|_{L^{2}(\mathcal{D})}$ defined by

$$
\operatorname{Std}\left(\|u\|_{L^{2}}\right)=\frac{\sqrt{\operatorname{Var}\|u\|_{L^{2}(\mathcal{D})}}}{\mathbb{E}\left[\|u\|_{L^{2}(\mathcal{D})}\right]} .
$$

The results for this estimator are shown on Table 18. Again, we see that the results provided by our approximation $u_{S}$ are very close to the reference results. The variance of the $L^{2}$ norm of the solution (in the sense of (3.13)) is therefore well-captured.

We thus see that our approach is more informative than the deterministic approach described above, as it provides accurate approximations not only in terms of $L^{2}$ and $H^{1}$ errors according to the estimators (3.1) but also, and this is important practically, for quantities of interest which are random in nature, such as (3.12) and (3.13). The additional cost of our approach consists in solving $M$ macroscopic problems (2.13) rather than 
TABLE 18. Estimator (3.13) for the test-case of Section 3.3.1 (left: $\kappa=73.61$ and $\zeta=1$; right: $\kappa=10$ and $\zeta=3)$.

\begin{tabular}{lccc}
\hline$\eta$ & $\operatorname{Std}\left(\left\|u_{\eta}^{\varepsilon}\right\|_{L^{2}}\right)$ & $\operatorname{Std}\left(\left\|u_{M}\right\|_{L^{2}}\right)$ & $\operatorname{Std}\left(\left\|u_{S}\right\|_{L^{2}}\right)$ \\
\hline 0.25 & $1.05 \times 10^{-3}$ & $1.11 \times 10^{-3}$ & $1.11 \times 10^{-3}$ \\
0.1 & $4.56 \times 10^{-4}$ & $4.82 \times 10^{-4}$ & $4.59 \times 10^{-4}$ \\
0.05 & $2.35 \times 10^{-4}$ & $2.48 \times 10^{-4}$ & $2.33 \times 10^{-4}$ \\
\hline
\end{tabular}

\begin{tabular}{lcc}
\hline$\eta$ & $\operatorname{Std}\left(\left\|u_{\eta}^{\varepsilon}\right\|_{L^{2}}\right)$ & $\operatorname{Std}\left(\left\|u_{S}\right\|_{L^{2}}\right)$ \\
\hline 0.25 & $4.47 \times 10^{-4}$ & $4.53 \times 10^{-4}$ \\
0.1 & $1.87 \times 10^{-4}$ & $1.84 \times 10^{-4}$ \\
0.05 & $9.51 \times 10^{-5}$ & $9.27 \times 10^{-5}$ \\
\hline
\end{tabular}

the unique macroscopic problem (3.11). However, as is standard for MsFEM approaches, the main cost of our approach is the offline cost, when constructing the highly oscillatory basis functions $\phi_{i}^{\varepsilon}$ of the approximation space $\mathcal{W}_{h}$. In comparison, the online cost, when solving (2.13), is very limited. Hence, our approach and the fully deterministic approach described above essentially share the same cost.

\section{ANALYSis}

This section is devoted to the analysis of the approach introduced in Section 2.2, and to the derivation of error bounds. As is often the case for the MsFEM (see e.g. [32]), we perform the analysis in a setting where the problem (1.2) - (1.3) that we consider admits a homogenized limit as $\varepsilon$ vanishes (although, we repeat it, the approach can be used in practice for more general cases, but is not supported by certified error bounds). The structure of our proof is similar to that for the deterministic setting, which we now overview (we refer to [32] for all the details).

In the case when the oversampling technique is not used, the MsFEM is a conforming Galerkin approximation, and the error is estimated using the Céa lemma:

$$
\left\|u^{\varepsilon}-u_{M}\right\|_{H^{1}} \leq C \inf _{v_{h} \in \mathcal{W}_{h}}\left\|u^{\varepsilon}-v_{h}\right\|_{H^{1}}
$$

where $u^{\varepsilon}$ is the solution to the reference deterministic highly oscillatory problem (1.1), $u_{M}$ is the MsFEM solution, and the constant $C$ is independent from $\varepsilon$ and $h$. On the other hand, when the oversampling technique is used, the MsFEM is a non-conforming Galerkin method. The error is then bounded from above by the sum of the best approximation error (the right-hand side of the above estimate) and the non-conforming error (that we do not detail here):

$$
\left\|u^{\varepsilon}-u_{M}\right\|_{H^{1}} \leq C\left[\inf _{v_{h} \in \mathcal{W}_{h}}\left\|u^{\varepsilon}-v_{h}\right\|_{H^{1}}+\text { non-conforming error }\right] .
$$

Note that, in the non-conforming case, the MsFEM solution $u_{M}$ does not belong to $H^{1}$, and one should write the above estimate with a broken $H^{1}$ norm and not the $H^{1}$ norm. For clarity, we ignore this distinction in this formal discussion.

Taking advantage of the homogenization setting, we introduce the two-scale expansion

$$
v^{\varepsilon}=u^{\star}+\varepsilon \sum_{i=1}^{d} w_{e_{i}}^{0}\left(\frac{\dot{\varepsilon}}{\varepsilon}\right) \partial_{i} u^{\star}
$$

of $u^{\varepsilon}$, where $u^{\star}$ is the homogenized solution, $w_{e_{i}}^{0}$ is the periodic corrector associated to $e_{i} \in \mathbb{R}^{d}$, and $\partial_{i} u^{\star}$ denotes the partial derivative $\frac{\partial u^{\star}}{\partial x_{i}}$. We next write

$$
\left\|u^{\varepsilon}-u_{M}\right\|_{H^{1}} \leq C\left[\left\|u^{\varepsilon}-v^{\varepsilon}\right\|_{H^{1}}+\inf _{v_{h} \in \mathcal{W}_{h}}\left\|v^{\varepsilon}-v_{h}\right\|_{H^{1}}+\text { non-conforming error }\right] .
$$


The first term in the right-hand side is estimated using standard homogenization results. To estimate the second term, one considers a suitably chosen element $v_{h} \in \mathcal{W}_{h}$, for which $\left\|v^{\varepsilon}-v_{h}\right\|_{H^{1}}$ can be estimated directly. The main idea is that the highly oscillating part of $v^{\varepsilon}$ can be well approached by an element in $\mathcal{W}_{h}$, since, by construction, the highly oscillatory basis functions are defined by a problem similar to the corrector problem, and thus encode the same highly oscillatory behavior as that present in the correctors $w_{e_{i}}^{0}$. We are thus left with approximating the slowly varying components of $v^{\varepsilon}$, for which standard FEM estimates are used. Lastly, we again use the fact that our problem admits a homogenized limit to estimate the third term, i.e. the non-conforming error.

In the sequel, we follow the same strategy in our stochastic setting. We hence first write (see (4.38) below) that

$$
\left\|u_{\eta}^{\varepsilon}(\cdot, \omega)-u_{S}(\cdot, \omega)\right\|_{H^{1}} \leq C\left[\inf _{v_{h} \in \mathcal{W}_{h}}\left\|u_{\eta}^{\varepsilon}(\cdot, \omega)-v_{h}(\cdot, \omega)\right\|_{H^{1}}+\text { non-conforming error }\right],
$$

where $u_{\eta}^{\varepsilon}$ is the solution to the reference stochastic problem (1.2)-(1.3) and $C$ is a deterministic constant independent from $\varepsilon, h$ and $\eta$ (note that, in (4.38), we use a broken $H^{1}$ norm rather than the $H^{1}$ norm; as pointed out above, this is due to the fact that our approach is a non-conforming Galerkin approximation; we ignore this distinction in the current discussion). To estimate the best approximation error (the first term in the right-hand side of (4.1) above), we use the triangle inequality, and write (see (4.58) below) that

$$
\inf _{v_{h} \in \mathcal{W}_{h}}\left\|u_{\eta}^{\varepsilon}(\cdot, \omega)-v_{h}(\cdot, \omega)\right\|_{H^{1}} \leq\left\|u_{\eta}^{\varepsilon}(\cdot, \omega)-v_{\eta}^{\varepsilon}(\cdot, \omega)\right\|_{H^{1}}+\inf _{v_{h} \in \mathcal{W}_{h}}\left\|v_{\eta}^{\varepsilon}(\cdot, \omega)-v_{h}(\cdot, \omega)\right\|_{H^{1}}
$$

where $v_{\eta}^{\varepsilon}$ is the two-scale expansion of the solution $u_{\eta}^{\varepsilon}$ truncated at order $\varepsilon^{2}$. A first difficulty owes to the fact that, in the general stochastic setting, no estimate is known on $\left\|u_{\eta}^{\varepsilon}(\cdot, \omega)-v_{\eta}^{\varepsilon}(\cdot, \omega)\right\|_{H^{1}}$. One only knows that its expectation vanishes when $\varepsilon \rightarrow 0$. However, in the present article, we consider a weakly stochastic case. In that setting, we have derived such a convergence rate type result in [42], and we can thus bound the first term of (4.2) (see Sect. 4.1.2 below for more details). The second term of (4.2), $\inf _{v_{h} \in \mathcal{W}_{h}}\left\|v_{\eta}^{\varepsilon}(\cdot, \omega)-v_{h}\right\|_{H^{1}}$, is estimated using an explicit construction of a suitable $v_{h}$ (see (4.59)), similarly to the deterministic setting. We again use there our specific weakly stochastic setting. Lastly, the non-conforming error (the second term in the right-hand side of (4.1) above) is estimated following arguments similar to those of the deterministic case, using that our problem admits a homogenized limit and is weakly stochastic.

This section is organized as follows. The error estimation is presented in Section 4.1. We first recall in Section 4.1.1 the formulation of the homogenized problem, and some results specific to the weakly stochastic case. Next, in Section 4.1.2, we establish an error bound between the reference solution $u_{\eta}^{\varepsilon}$ and its two-scale expansion $v_{\eta}^{\varepsilon}$ (see Thm. 4.2), which allows to bound the first term in the right-hand side of (4.2). Our main result, Theorem 4.5, is given in Section 4.1.3, and proved in Section 4.2. The proof essentially consists in explicitly building a function $v_{h} \in \mathcal{W}_{h}$ such that the second term of (4.2) can be directly estimated. It also makes use of several technical results (Lems. 4.8, 4.9 and 4.11 below) to bound the non-conforming error, i.e. the second term in the right hand side of (4.1). The proof of these technical results is postponed until Appendix A. Last, in Section 4.3, we specifically consider the one dimensional case.

Before proceeding further, we recall the setting of stochastic homogenization we work with. The reader familiar with this theory may directly proceed to Section 4.1 . Let $(\Omega, \mathcal{F}, \mathbb{P})$ be a probability space. For a random variable $X \in L^{1}(\Omega, d \mathbb{P})$, we denote by $\mathbb{E}(X)=\int_{\Omega} X(\omega) d \mathbb{P}(\omega)$ its expectation value. We assume that the group $\left(\mathbb{Z}^{d},+\right)$ acts on $\Omega$. We denote by $\left(\tau_{k}\right)_{k \in \mathbb{Z}^{d}}$ this action, and assume that it preserves the measure $\mathbb{P}$, i.e.

$$
\forall k \in \mathbb{Z}^{d}, \quad \forall A \in \mathcal{F}, \quad \mathbb{P}\left(\tau_{k} A\right)=\mathbb{P}(A) .
$$

We assume that $\tau$ is ergodic, that is,

$$
\forall A \in \mathcal{F}, \quad\left(\forall k \in \mathbb{Z}^{d}, \quad \tau_{k} A=A\right) \Rightarrow(\mathbb{P}(A)=0 \quad \text { or } \quad 1) .
$$


We define the following notion of stationarity: any $F \in L_{\mathrm{loc}}^{1}\left(\mathbb{R}^{d}, L^{1}(\Omega)\right)$ is said to be stationary if

$$
\forall k \in \mathbb{Z}^{d}, \quad F(x+k, \omega)=F\left(x, \tau_{k} \omega\right) \text { almost everywhere, almost surely. }
$$

Note that we have chosen to present the theory in a discrete stationary setting, which is more appropriate for our specific purpose, which is to consider a setting close to periodic homogenization. Random homogenization is more often presented in the continuous stationary setting. This is only a matter of small modifications. We refer to the bibliography for the latter.

For the sake of analysis, we assume in this section that the matrix $A_{\eta}^{\varepsilon}(x, \omega)$ in $(1.2)-(1.3)$ reads $A_{\eta}^{\varepsilon}(x, \omega)=$ $A_{\eta}\left(\frac{x}{\varepsilon}, \omega\right)$, where the random matrix $A_{\eta}$ is stationary in the sense of (4.3). The problem (1.2) now reads

$$
-\operatorname{div}\left[A_{\eta}\left(\frac{\dot{-}}{\varepsilon}, \omega\right) \nabla u_{\eta}^{\varepsilon}(\cdot, \omega)\right]=f \text { in } \mathcal{D}, \quad u_{\eta}^{\varepsilon}(\cdot, \omega)=0 \quad \text { on } \partial \mathcal{D},
$$

where $A_{\eta}(\cdot, \omega) \in\left(L^{\infty}\left(\mathbb{R}^{d}\right)\right)^{d \times d}$ satisfies the standard coercivity and boundedness conditions: there exist two deterministic constants $a_{+} \geq a_{-}>0$ such that

$$
\forall \eta, \forall \xi \in \mathbb{R}^{d}, \quad a_{-}|\xi|^{2} \leq A_{\eta}(x, \omega) \xi \cdot \xi \text { a.e. on } \mathbb{R}^{d} \text {, a.s. and }\left\|A_{\eta}(\cdot, \omega)\right\|_{L^{\infty}\left(\mathbb{R}^{d}\right)} \leq a_{+} \text {a.s. }
$$

Due to the stationarity assumption on $A_{\eta}$, the problem (4.4) admits a homogenized limit when $\varepsilon \rightarrow 0$. Note that, to the best of our knowledge, all analyses of the MsFEM approach in the deterministic setting that have been proposed in the literature are performed under a similar assumption (the matrix $A^{\varepsilon}$ in (1.1) is assumed to read $A^{\varepsilon}(x)=A_{\text {per }}\left(\frac{x}{\varepsilon}\right)$ for a fixed periodic matrix $A_{\text {per }}$, see e.g. $\left.[32,38]\right)$.

In addition, in line with (1.3) and (2.12), we assume that $A_{\eta}$ is of the form

$$
A_{\eta}(x, \omega)=A_{\text {per }}(x)+\eta A_{1}(x, \omega),
$$

where $\eta \in \mathbb{R}$ is small parameter (we henceforth assume that $|\eta| \leq 1$ ), $A_{\text {per }}$ is a symmetric bounded $Q$-periodic matrix $\left(Q=[0,1]^{d}\right)$ satisfying the ellipticity condition almost everywhere on $\mathbb{R}^{d}$, and $A_{1}$ is a symmetric bounded stationary matrix: $\left|A_{1}(x, \omega)\right| \leq C$ almost everywhere in $\mathbb{R}^{d}$, almost surely. Since $\eta$ is small, our problem is weakly stochastic.

In line with (3.5), we furthermore assume that $A_{1}$ is of the form

$$
A_{1}(x, \omega)=\sum_{k \in \mathbb{Z}^{d}} \mathbf{1}_{Q+k}(x) X_{k}(\omega) B_{\mathrm{per}}(x),
$$

where $\left(X_{k}(\omega)\right)_{k \in \mathbb{Z}^{d}}$ is a sequence of i.i.d. scalar random variables such that

$$
\exists C, \forall k \in \mathbb{Z}^{d}, \quad\left|X_{k}(\omega)\right| \leq C \quad \text { almost surely, }
$$

and $B_{\text {per }} \in\left(L^{\infty}\left(\mathbb{R}^{d}\right)\right)^{d \times d}$ is a $Q$-periodic matrix. Besides being used in Theorem 4.2 below, this assumption is also used in the proof of Lemma 4.11, to recognize that some quantity (namely, (A.16) below) is a normalized sum of i.i.d. variables, on which we can use Central Limit Theorem arguments. As mentioned in Section 3.1 above, the form (4.7) is not essential. The point in (4.7) is that $A_{1}$ is a sum of direct products of a function depending on $x$ with a random variable only depending on $\omega$. Assumptions alternative to (4.7) could be made, that still satisfy this framework.

Finally, we assume that

$A_{\text {per }}$ is Hölder continuous,

$B_{\text {per }}$ is Hölder continuous. 
We use these assumptions to obtain a rate of convergence of the two-scale expansion of $u_{\eta}^{\varepsilon}$ (see [42] and Thm. 4.2 below), and hence control the first term in the right-hand side of (4.2). Such assumptions are standard when proving convergence rates of two-scale expansions (see e.g. [40], p. 28). In turn, to control the second term in (4.2) and the non-conforming error (the second term in (4.1)), we do not need $B_{\text {per }}$ to be Hölder continuous, and only use the fact that $A_{\text {per }}$ is Hölder continuous (to obtain e.g. Lems. 4.4, 4.8, 4.9 and 4.12). The numerical examples that we have considered in Section 3 satisfy assumptions (4.8)-(4.9) (remark that assumption (4.8) is also satisfied in the numerical examples considered in e.g. [30]).

Note that we have assumed $A_{\text {per }}$ and $B_{\text {per }}$ to be symmetric only for the sake of simplicity. The arguments used below carry over to the non-symmetric case up to slight modifications.

\subsection{Error estimation}

To bound the error between the reference solution $u_{\eta}^{\varepsilon}$ and the MsFEM solution $u_{S}$, we use in many instances that we work in a weakly stochastic homogenization setting. We first recall in Section 4.1.1 some results specific to weakly stochastic homogenization. This setting also allows to state rates of convergence for the two-scale expansion of $u_{\eta}^{\varepsilon}$, as we explain in Section 4.1.2. Our main result, Theorem 4.5, is given in Section 4.1.3.

\subsubsection{The homogenized equation}

Under the conditions recalled above, it is known (see e.g. $[12,40])$ that the solution $u_{\eta}^{\varepsilon}(\cdot, \omega)$ to $(4.4)$ a.s. converges weakly in $H_{0}^{1}(\mathcal{D})$ as $\varepsilon \rightarrow 0$ to the deterministic solution $u_{\eta}^{\star}$ of the homogenized equation

$$
-\operatorname{div}\left[A_{\eta}^{\star} \nabla u_{\eta}^{\star}\right]=f \quad \text { in } \mathcal{D}, \quad u_{\eta}^{\star}=0 \quad \text { on } \partial \mathcal{D} .
$$

The homogenized matrix is given by

$$
\left(A_{\eta}^{\star}\right)_{i j}=\mathbb{E}\left(\int_{Q}\left(e_{i}+\nabla w_{e_{i}}^{\eta}(y, \cdot)\right)^{T} A_{\eta}(y, \cdot)\left(e_{j}+\nabla w_{e_{j}}^{\eta}(y, \cdot)\right) \mathrm{d} y\right),
$$

where, for any $p \in \mathbb{R}^{d}, w_{p}^{\eta}$ is the unique (up to the addition of a random constant) solution to the corrector problem

$$
\left\{\begin{array}{l}
-\operatorname{div}\left[A_{\eta}(\cdot, \omega)\left(p+\nabla w_{p}^{\eta}(\cdot, \omega)\right)\right]=0 \quad \text { in } \mathbb{R}^{d}, \\
\nabla w_{p}^{\eta} \text { is stationary in the sense of }(4.3), \quad \mathbb{E}\left(\int_{Q} \nabla w_{p}^{\eta}(y, \cdot) \mathrm{d} y\right)=0 .
\end{array}\right.
$$

The variational problem associated with (4.10) writes: find $u_{\eta}^{\star} \in H_{0}^{1}(\mathcal{D})$ such that

$$
\forall v \in H_{0}^{1}(\mathcal{D}), \quad \mathcal{A}_{\eta}^{\star}\left(u_{\eta}^{\star}, v\right)=b(v),
$$

where

$$
\mathcal{A}_{\eta}^{\star}(u, v)=\int_{\mathcal{D}}(\nabla v(x))^{T} A_{\eta}^{\star} \nabla u(x) \mathrm{d} x \quad \text { and } \quad b(v)=\int_{\mathcal{D}} f(x) v(x) \mathrm{d} x .
$$

As shown in $[13,24]$, in the weakly stochastic setting, the homogenized matrix $A_{\eta}^{\star}$ can be expanded in terms of a series in powers of $\eta$ :

$$
A_{\eta}^{\star}=A_{\mathrm{per}}^{\star}+\eta A_{1}^{\star}+\eta^{2} A_{2}^{\star}(\eta),
$$

where $A_{2}^{\star}(\eta)$ is a deterministic matrix, that depends on $\eta$ and is bounded as $\eta \rightarrow 0$, and where, for any $1 \leq i, j \leq d$,

$$
\begin{aligned}
\left(A_{\mathrm{per}}^{\star}\right)_{i j} & =\int_{Q}\left(e_{i}+\nabla w_{e_{i}}^{0}\right)^{T} A_{\mathrm{per}}\left(e_{j}+\nabla w_{e_{j}}^{0}\right), \\
\left(A_{1}^{\star}\right)_{i j} & =\int_{Q}\left(e_{i}+\nabla w_{e_{i}}^{0}\right)^{T} \mathbb{E}\left(A_{1}\right)\left(e_{j}+\nabla w_{e_{j}}^{0}\right),
\end{aligned}
$$


where, for any $p \in \mathbb{R}^{d}, w_{p}^{0}$ is the unique (up to the addition of a constant) solution to the deterministic corrector problem associated to the periodic matrix $A_{\text {per }}$ :

$$
-\operatorname{div}\left[A_{\mathrm{per}}\left(p+\nabla w_{p}^{0}\right)\right]=0, \quad w_{p}^{0} \text { is } Q \text {-periodic. }
$$

Under the assumption (4.7), we have $A_{1}^{\star}=\mathbb{E}\left(X_{0}\right) \bar{B}$, with

$$
\forall 1 \leq i, j \leq d, \quad \bar{B}_{i j}=\int_{Q}\left(e_{i}+\nabla w_{e_{i}}^{0}\right)^{T} B_{\mathrm{per}}\left(e_{j}+\nabla w_{e_{j}}^{0}\right) .
$$

Remark 4.1. In general, when $A_{\text {per }}$ is not symmetric, the expression of $A_{1}^{\star}$ includes additional terms. Indeed, writing $\nabla w_{p}^{\eta}=\nabla w_{p}^{0}+\eta \nabla w_{p}^{1}+O\left(\eta^{2}\right)$, we in general need $\mathbb{E}\left(\nabla w_{p}^{1}\right)$ to compute $A_{1}^{\star}$ (see e.g. $\left.[13,24]\right)$. In the symmetric case, these additional terms vanish, see e.g. Remark 4.2, page 117 of [4]. In the non-symmetric case, the expression (4.16) of $A_{1}^{\star}$ needs to be slightly modified, but the expansion (4.14) remains true. Our arguments hence carry over to the non-symmetric case.

Using the expansion (4.14) of $A_{\eta}^{\star}$ with respect to $\eta$, it is easy to see that the solution $u_{\eta}^{\star}$ to (4.10) can also be expanded in a series in powers of $\eta$. We have

$$
u_{\eta}^{\star}=u_{0}^{\star}+\eta \mathbb{E}\left(X_{0}\right) \bar{u}_{1}^{\star}+\eta^{2} r_{\eta} \quad \text { with } \quad\left\|r_{\eta}\right\|_{H^{1}(\mathcal{D})} \leq C,
$$

where $C$ is a constant independent of $\eta$, and where $u_{0}^{\star}$ solves

$$
-\operatorname{div}\left[A_{\mathrm{per}}^{\star} \nabla u_{0}^{\star}\right]=f \text { in } \mathcal{D}, \quad u_{0}^{\star}=0 \text { on } \partial \mathcal{D},
$$

and $\bar{u}_{1}^{\star}$ solves

$$
-\operatorname{div}\left[A_{\mathrm{per}}^{\star} \nabla \bar{u}_{1}^{\star}\right]=\operatorname{div}\left[\bar{B} \nabla u_{0}^{\star}\right] \quad \text { in } \mathcal{D}, \quad \bar{u}_{1}^{\star}=0 \quad \text { on } \partial \mathcal{D} .
$$

The expansion (4.19) will be useful in the sequel. We will also need a bound on $u_{\eta}^{\star}$ and $r_{\eta}$ in the $H^{2}$ norm. Recall that $u_{\eta}^{\star}$ is the solution to (4.10), whereas $r_{\eta}$ is solution to

$$
-\operatorname{div}\left[A_{\eta}^{\star} \nabla r_{\eta}\right]=\operatorname{div}\left[A_{2}^{\star}(\eta)\left(\nabla u_{0}^{\star}+\eta \mathbb{E}\left(X_{0}\right) \nabla \bar{u}_{1}^{\star}\right)+\mathbb{E}\left(X_{0}\right) A_{1}^{\star} \nabla \bar{u}_{1}^{\star}\right] \quad \text { in } \mathcal{D}, \quad r_{\eta}=0 \quad \text { on } \partial \mathcal{D} .
$$

In view of (4.5), we have, almost surely and almost everywhere, $a_{-} \operatorname{Id} \leq A_{\eta} \leq a_{+}$Id in the sense of symmetric matrices. Recalling that homogenization preserves the order of symmetric matrices (see e.g. [48], p. 12), we deduce that

$$
\forall \eta, \forall \xi \in \mathbb{R}^{d}, \quad a_{-}|\xi|^{2} \leq A_{\eta}^{\star} \xi \cdot \xi \leq a_{+}|\xi|^{2} .
$$

In addition, the right-hand sides of (4.10) and (4.22) are bounded uniformly in $\eta$ in the $L^{2}$ norm. Using Theorems 9.15 and 9.14 of [34], we obtain that there exists $C$ such that

$$
\forall \eta, \quad\left\|u_{\eta}^{\star}\right\|_{H^{2}(\mathcal{D})} \leq C \text { and } \quad\left\|r_{\eta}\right\|_{H^{2}(\mathcal{D})} \leq C .
$$

\subsubsection{Two scale expansion of the reference solution $u_{\eta}^{\varepsilon}$}

As recalled above, the standard error analysis for the MsFEM in the deterministic setting is performed in the case when the matrix $A^{\varepsilon}$ in (1.1) reads $A^{\varepsilon}(x) \equiv A_{\text {per }}(x / \varepsilon)$ for a fixed periodic matrix $A_{\text {per. }}$ The problem (1.1) then admits a homogenized limit. To obtain bounds on the MsFEM error, one step of the proof is to approximate the oscillatory solution $u^{\varepsilon}$ by its two-scale expansion $u^{\star}+\varepsilon \sum_{i=1}^{d} w_{e_{i}}^{0}\left(\frac{\dot{\varepsilon}}{\varepsilon}\right) \partial_{i} u^{\star}$, where $u^{\star}$ is the homogenized solution, $w_{p}^{0}$ is the periodic corrector associated to $p \in \mathbb{R}^{d}$, and $\partial_{i} u^{\star}=\frac{\partial u^{\star}}{\partial x_{i}}$. In the deterministic case, it is known (see e.g. $[12,23,40]$ ) that, under some regularity assumptions on $A_{\text {per }}$ and $u^{\star}$,

$$
\left\|u^{\varepsilon}-\left[u^{\star}+\varepsilon \sum_{i=1}^{d} w_{e_{i}}^{0}\left(\frac{\dot{\varepsilon}}{\varepsilon}\right) \partial_{i} u^{\star}\right]\right\|_{H^{1}(\mathcal{D})} \leq C \sqrt{\varepsilon}
$$

for a constant $C$ independent of $\varepsilon$. 
In the stochastic case, it is known that $\mathbb{E}\left[\left\|u^{\varepsilon}-\left[u^{\star}+\varepsilon \sum_{i=1}^{d} w_{e_{i}}\left(\frac{\cdot}{\varepsilon}, \omega\right) \partial_{i} u^{\star}\right]\right\|_{H^{1}(\mathcal{D})}^{2}\right]$ converges to 0 as $\varepsilon \rightarrow 0$ (see [47], Thm. 3), but no rate of convergence is known (except in some one-dimensional situations, see e.g. $[10,16,43])$. However, in the present article, and as announced above, we consider a weakly stochastic case. In this setting, we have derived in [42] a result similar to (4.24). We now state this result, which will be useful for our analysis.

Theorem 4.2 (from [42], Thm. 2). Assume $d>1$. Let $u_{\eta}^{\varepsilon}$ be the solution to (4.4), and assume that $A_{\eta}$ satisfies (4.6)-(4.7)-(4.8)-(4.9). Let $A_{\mathrm{per}}^{\star}, w_{p}^{0}, u_{0}^{\star}$ and $\bar{u}_{1}^{\star}$ be defined by (4.15), (4.17), (4.20) and (4.21). The two-scale expansion $v_{\eta}^{\varepsilon}$ of $u_{\eta}^{\varepsilon}$ reads

$$
\begin{aligned}
v_{\eta}^{\varepsilon}(\cdot, \omega)= & u_{0}^{\star}+\eta \mathbb{E}\left(X_{0}\right) \bar{u}_{1}^{\star}+\varepsilon \sum_{i=1}^{d}\left[w_{e_{i}}^{0}\left(\frac{\dot{\varepsilon}}{\varepsilon}\right)\left(\partial_{i} u_{0}^{\star}+\eta \mathbb{E}\left(X_{0}\right) \partial_{i} \bar{u}_{1}^{\star}\right)\right. \\
& \left.+\eta \mathbb{E}\left(X_{0}\right) \psi_{e_{i}}\left(\frac{\dot{z}}{\varepsilon}\right) \partial_{i} u_{0}^{\star}+\eta \sum_{k \in I_{\varepsilon}}\left(X_{k}(\omega)-\mathbb{E}\left(X_{0}\right)\right) \chi_{e_{i}}\left(\frac{\dot{\varepsilon}}{\varepsilon}-k\right) \partial_{i} u_{0}^{\star}\right],
\end{aligned}
$$

where

$$
I_{\varepsilon}=\left\{k \in \mathbb{Z}^{d} \text { such that } \varepsilon(Q+k) \cap \mathcal{D} \neq \emptyset\right\},
$$

and where, for any $p \in \mathbb{R}^{d}, \psi_{p}$ is the solution (unique up to the addition of a constant) to

$$
-\operatorname{div}\left[A_{\mathrm{per}} \nabla \psi_{p}\right]=\operatorname{div}\left[B_{\mathrm{per}}\left(p+\nabla w_{p}^{0}\right)\right], \quad \psi_{p} \text { is } Q \text {-periodic, }
$$

and $\chi_{p}$ is the unique solution to

$$
\left\{\begin{array}{l}
-\operatorname{div}\left[A_{\mathrm{per}} \nabla \chi_{p}\right]=\operatorname{div}\left[\mathbf{1}_{Q} B_{\mathrm{per}}\left(p+\nabla w_{p}^{0}\right)\right] \quad \text { in } \mathbb{R}^{d}, \\
\chi_{p} \in L_{l o c}^{2}\left(\mathbb{R}^{d}\right), \quad \nabla \chi_{p} \in\left(L^{2}\left(\mathbb{R}^{d}\right)\right)^{d}, \quad \lim _{|x| \rightarrow \infty} \chi_{p}(x)=0 .
\end{array}\right.
$$

We assume that $u_{0}^{\star} \in W^{2, \infty}(\mathcal{D})$ and $\bar{u}_{1}^{\star} \in W^{2, \infty}(\mathcal{D})$. Then

$$
\sqrt{\mathbb{E}\left[\left\|u_{\eta}^{\varepsilon}-v_{\eta}^{\varepsilon}\right\|_{H^{1}(\mathcal{D})}^{2}\right]} \leq C\left(\sqrt{\varepsilon}+\eta \sqrt{\varepsilon \ln (1 / \varepsilon)}+\eta^{2}\right)
$$

where $C$ is a constant independent of $\varepsilon$ and $\eta$.

As pointed out above and in [42], the assumptions (4.8)-(4.9) are standard assumptions when proving convergence rates of two-scale expansions (see e.g. [40], p. 28). Likewise, the assumption $u_{0}^{\star} \in W^{2, \infty}(\mathcal{D})$ (and subsequently $\bar{u}_{1}^{\star} \in W^{2, \infty}(\mathcal{D})$ ) is a standard assumption (see e.g. [2], Thm. 2.1 and [40], p. 28). In view of (4.20), this assumption implies that the right hand side $f$ in $(4.4)$ belongs to $L^{\infty}(\mathcal{D})$.

In dimension $d=1$, the boundary conditions of (4.27) need to be modified for this problem to have a solution. We have derived in [42] the following result, which is the one-dimensional version of Theorem 4.2 (note that we need below weaker assumptions than in Theorem 4.2, as pointed out in [42]: we do not need to assume (4.8)-(4.9), and the assumption $f \in L^{2}(\mathcal{D})$ is enough):

Theorem 4.3 (from [42], Theorem 3). Assume that the dimension $d$ is equal to one. Let $u_{\eta}^{\varepsilon}$ be the solution to (4.4) in the domain $\mathcal{D}$ with $f \in L^{2}(\mathcal{D})$, and assume that $A_{\eta}$ satisfies $(4.6)-(4.7)$. Let $v_{\eta}^{\varepsilon}$ be defined by (4.25), where the definition (4.27) is replaced by

$$
\left\{\begin{array}{l}
-\left[A_{\mathrm{per}} \chi^{\prime}\right]^{\prime}=\left[\mathbf{1}_{(0,1)} B_{\mathrm{per}}\left(1+\left(w^{0}\right)^{\prime}\right)\right]^{\prime} \text { in } \mathbb{R} \\
\chi \in L_{l o c}^{2}(\mathbb{R}), \quad \chi^{\prime} \in L^{2}(\mathbb{R})
\end{array}\right.
$$


where $w^{0}$ solves (4.17). Then

$$
\sqrt{\mathbb{E}\left[\left\|u_{\eta}^{\varepsilon}-v_{\eta}^{\varepsilon}\right\|_{L^{\infty}(\mathcal{D})}^{2}\right]}+\sqrt{\mathbb{E}\left[\left\|u_{\eta}^{\varepsilon}-v_{\eta}^{\varepsilon}\right\|_{H^{1}(\mathcal{D})}^{2}\right]} \leq C\left(\varepsilon+\eta \sqrt{\varepsilon}+\eta^{2}\right),
$$

where $C$ is a constant independent of $\varepsilon$ and $\eta$.

The following estimate, which is established in the proof of Proposition 11 of [42] and useful to demonstrate (4.28), will also be useful here:

Lemma 4.4 (from [42], proof of Prop. 11). We assume (4.8) and $d>1$. For any $p \in \mathbb{R}^{d}$, any $k \in \mathbb{Z}^{d}$, and any bounded domain $\mathcal{D} \subset \mathbb{R}^{d}$, the function $\chi_{p}$ defined by (4.27) satisfies

$$
\left\|\chi_{p}\left(\frac{\dot{\varepsilon}}{\varepsilon}-k\right)\right\|_{L^{2}(\mathcal{D})}^{2} \leq C \varepsilon^{d} R_{d, \varepsilon}
$$

for a constant $C$ independent of $k$ and $\varepsilon$, where $R_{d, \varepsilon}=1$ if $d>2$, and $R_{d, \varepsilon}=1+\ln (1 / \varepsilon)$ if $d=2$.

\subsubsection{Main result}

Before presenting our main result, we need some useful notation. Following the approach presented in Section 2.2 , we recall that

$$
\mathcal{W}_{h}:=\operatorname{span}\left(\phi_{i}^{\varepsilon}, i=1, \ldots, L\right),
$$

where $\phi_{i}^{\varepsilon}$ are the highly oscillatory MsFEM basis functions. By construction, the solution $u_{S} \in \mathcal{W}_{h}$ of the weak stochastic MsFEM approach (2.13) satisfies

$$
\forall v_{h} \in \mathcal{W}_{h}, \quad \mathcal{A}_{\varepsilon, \eta}^{h}\left(u_{S}, v_{h}\right)=b\left(v_{h}\right) \quad \text { a.s. }
$$

where, for any $u$ and $v$ in $\mathcal{W}_{h}$,

$$
\mathcal{A}_{\varepsilon, \eta}^{h}(u, v)=\sum_{\mathbf{K} \in \mathcal{T}_{h}} \int_{\mathbf{K}}(\nabla v(x))^{T} A_{\eta}\left(\frac{x}{\varepsilon}, \omega\right) \nabla u(x) \mathrm{d} x \quad \text { and } \quad b(v)=\int_{\mathcal{D}} f(x) v(x) \mathrm{d} x .
$$

For future use, we also define, on the standard finite element space

$$
\mathcal{V}_{h}:=\operatorname{span}\left(\phi_{i}^{0}, i=1, \ldots, L\right)
$$

the forms

$$
\widetilde{\mathcal{A}}_{\varepsilon, \eta}^{h}(u, v)=\sum_{\mathbf{K} \in \mathcal{T}_{h}} \int_{\mathbf{K}}\left(\nabla\left(\mathcal{R}_{\mathbf{K}}^{\varepsilon}(v)\right)(x)\right)^{T} A_{\eta}\left(\frac{x}{\varepsilon}, \omega\right) \nabla\left(\mathcal{R}_{\mathbf{K}}^{\varepsilon}(u)\right)(x) \mathrm{d} x, \quad \widetilde{b}_{h}(v)=\sum_{\mathbf{K} \in \mathcal{T}_{h}} \int_{\mathbf{K}} f(x) \mathcal{R}_{\mathbf{K}}^{\varepsilon}(v)(x) \mathrm{d} x,
$$

where the local, linear operators $\mathcal{R}_{\mathbf{K}}^{\varepsilon}$ are defined on $\mathcal{V}_{h}$ by

$$
\forall 1 \leq i \leq L, \quad \mathcal{R}_{\mathbf{K}}^{\varepsilon}\left(\left.\phi_{i}^{0}\right|_{\mathbf{K}}\right)=\left.\phi_{i}^{\varepsilon}\right|_{\mathbf{K}} .
$$

These local operators give rise to the global operator $\mathcal{R}^{\varepsilon}: \mathcal{V}_{h} \rightarrow \mathcal{W}_{h}$ defined by

$$
\forall \mathbf{K}, \quad \forall v \in \mathcal{V}_{h},\left.\quad \mathcal{R}^{\varepsilon}(v)\right|_{\mathbf{K}}=\mathcal{R}_{\mathbf{K}}^{\varepsilon}\left(\left.v\right|_{\mathbf{K}}\right) .
$$

As pointed out above, the space $\mathcal{W}_{h}$ is not a subspace of $H_{0}^{1}(\mathcal{D})$, as the basis functions $\phi_{i}^{\varepsilon}$ may have jumps at the finite element boundaries (due to the use of the oversampling technique). We will hence work with the broken $H^{1}$-norm introduced in (3.2), that reads, we recall,

$$
\forall v_{h} \in \mathcal{W}_{h}, \quad\left\|v_{h}\right\|_{H_{h}^{1}}=\left[\sum_{\mathbf{K} \in \mathcal{T}_{h}}\left\|v_{h}\right\|_{H^{1}(\mathbf{K})}^{2}\right]^{1 / 2} .
$$


We are now in position to present the main result of this article. We introduce the notation $Q_{i}^{\varepsilon}=\varepsilon(i+Q)$ for any $i \in \mathbb{Z}^{d}$, and denote by $N_{\mathbf{K}}$ the number of cells $Q_{i}^{\varepsilon}$ in the element $\mathbf{K}: N_{\mathbf{K}}=\operatorname{Card}\left(i ; Q_{i}^{\varepsilon} \subset \mathbf{K}\right)$. We make in the theorem below a regularity hypothese on the macroscopic mesh, assuming that the volume of each element is bounded from below by $\alpha h^{d}$, for some $\alpha>0$, and hence that $N_{\mathbf{K}} \geq \alpha(h / \varepsilon)^{d}$.

Theorem 4.5. Assume that $A_{\eta}$ satisfies (4.6)-(4.7)-(4.8)-(4.9). We assume that $u_{0}^{\star}$ and $\bar{u}_{1}^{\star}$ respectively defined by (4.20) and (4.21) satisfy $u_{0}^{\star} \in W^{2, \infty}(\mathcal{D})$ and $\bar{u}_{1}^{\star} \in W^{2, \infty}(\mathcal{D})$. Let $u_{\eta}^{\varepsilon}$ be the solution to (4.4) and $u_{S}$ be the weakly stochastic MsFEM solution to (4.32). Suppose that $d>1, \varepsilon \leq h,|\eta| \leq 1$, and that there exists $\alpha>0$, independent of $\mathbf{K}, h$ and $\varepsilon$, such that $N_{\mathbf{K}} \geq \alpha\left(\frac{h}{\varepsilon}\right)^{d}$. We then have

$$
\sqrt{\mathbb{E}\left[\left\|u_{\eta}^{\varepsilon}-u_{S}\right\|_{H_{h}^{1}}^{2}\right]} \leq C\left(\sqrt{\varepsilon}+h+\frac{\varepsilon}{h}+\eta\left(\frac{\varepsilon}{h}\right)^{d / 2} \ln (N(h))+\eta+\eta^{2} \mathcal{C}(\eta)\right),
$$

where $C$ is a constant independent of $\varepsilon, h$ and $\eta, N(h)$ is the number of elements $\mathbf{K}$ in the domain $\mathcal{D}$ (which is of order $h^{-d}$ in dimension $\left.d\right)$, and $\mathcal{C}$ is a bounded function as $\eta$ goes to 0.

The restriction to $d>1$ comes from the fact that the proof of this result uses the rate of convergence on the two-scale expansion of $u_{\eta}^{\varepsilon}$ that we stated in Theorem 4.2. This rate of convergence is not optimal in dimension one, as can be seen from the comparison of (4.28) and (4.30). The one-dimensional version of the above result is stated in Section 4.3 below (see Thm. 4.13), where we briefly consider the one-dimensional situation.

Remark 4.6. In the case $\eta=0$, our approach reduces to the standard deterministic MsFEM and we obtain the same estimate as in the deterministic case with oversampling (see e.g. [32], Thm. 3.1). Note also that the linear dependency of the estimate with respect to $\eta$ is consistent with the numerical results of Section 3 (at least when parameters are chosen such that the error associated to $\eta$ dominates the overall error).

\subsection{Proof of Theorem 4.5}

The proof of Theorem 4.5 is the direct consequence of three lemmas. First we recall the second Strang's Lemma (see e.g. Thm. 4.2.2, p. 210 of [22]).

Lemma 4.7. Consider a family of Hilbert spaces $\mathcal{W}_{h}$ with the norm $\|\cdot\|_{H_{h}^{1}}$, a family of continuous bilinear forms $\mathcal{A}_{\varepsilon, \eta}^{h}$ on $\mathcal{W}_{h}$ that are uniformly $\mathcal{W}_{h}$-elliptic, and a continuous linear form $b$ on $\mathcal{W}_{h}$. For any $h>0$, introduce $u_{S}$ solution to

and $u_{\eta}^{\varepsilon} \in H_{0}^{1}(\mathcal{D})$ solution to

$$
\forall v_{h} \in \mathcal{W}_{h}, \quad \mathcal{A}_{\varepsilon, \eta}^{h}\left(u_{S}, v_{h}\right)=b\left(v_{h}\right)
$$

$$
\forall v \in H_{0}^{1}(\mathcal{D}), \quad \mathcal{A}_{\varepsilon, \eta}^{h}\left(u_{\eta}^{\varepsilon}, v\right)=b(v) .
$$

Then there exists a constant $C$ independent of $\eta, h$ and $\varepsilon$ such that

$$
\left\|u_{\eta}^{\varepsilon}-u_{S}\right\|_{H_{h}^{1}} \leq C\left(\inf _{v_{h} \in \mathcal{W}_{h}}\left\|u_{\eta}^{\varepsilon}-v_{h}\right\|_{H_{h}^{1}}+\sup _{w_{h} \in \mathcal{W}_{h}} \frac{\left|\mathcal{A}_{\varepsilon, \eta}^{h}\left(u_{\eta}^{\varepsilon}, w_{h}\right)-b\left(w_{h}\right)\right|}{\left\|w_{h}\right\|_{H_{h}^{1}}}\right) .
$$

The first term in the right hand side of (4.38) is the so-called best approximation error. The main part (Step 2) of the proof of Theorem 4.5 is devoted to its estimation, following up on the estimate (4.28) provided by Theorem 4.2 .

The second term in the right hand side of (4.38) is the so-called nonconforming error, which vanishes in the case $\mathcal{W}_{h} \subset H_{0}^{1}(\mathcal{D})$ (the method is then conforming, and we are left with the standard Céa lemma). In our case, we use the oversampling technique, hence our approximation is not conforming, and this second term does not vanish. It will be estimated in the step 3 of the proof of Theorem 4.5, using the following two results, which are proved in Appendix A. 
Lemma 4.8. Consider the two bilinear forms $\mathcal{A}_{\eta}^{\star}$ and $\widetilde{\mathcal{A}}_{\varepsilon, \eta}^{h}$ respectively defined in (4.13) and (4.34). Under assumption (4.8), there exists a deterministic constant $C$, independent of $\eta$, $\varepsilon$ and $h$, such that, for any $v_{h} \in \mathcal{V}_{h}$,

$$
\sup _{w_{h} \in \mathcal{V}_{h}} \frac{\left|\widetilde{\mathcal{A}}_{\varepsilon, \eta}^{h}\left(v_{h}, w_{h}\right)-\mathcal{A}_{\eta}^{\star}\left(v_{h}, w_{h}\right)\right|}{\left\|w_{h}\right\|_{H^{1}(\mathcal{D})}} \leq C\left(\frac{\varepsilon}{h}+\eta \lambda(\omega, h, \varepsilon)+\eta^{2} \mathcal{C}(\eta)\right)\left\|v_{h}\right\|_{H^{1}(\mathcal{D})} \quad \text { a.s. },
$$

where $\mathcal{C}$ is a deterministic function independent of $\varepsilon$ and $h$ and bounded when $\eta \rightarrow 0$, and $\lambda$ is defined by

$$
\lambda(\omega, h, \varepsilon)=\max _{\mathbf{K}} \max _{1 \leq p, m \leq d}\left|\frac{1}{\left|I_{\mathbf{K}}^{\varepsilon}\right|} \int_{I_{\mathbf{K}}^{\varepsilon}}\left[e_{p}+\nabla w_{e_{p}}^{0}\left(\frac{x}{\varepsilon}\right)\right]^{T}\left[A_{1}\left(\frac{x}{\varepsilon}, \omega\right)-\mathbb{E}\left(A_{1}\left(\frac{x}{\varepsilon}, \cdot\right)\right)\right]\left[e_{m}+\nabla w_{e_{m}}^{0}\left(\frac{x}{\varepsilon}\right)\right] \mathrm{d} x\right|
$$

where $I_{\mathbf{K}}^{\varepsilon}$ is the largest domain composed of cells of size $\varepsilon$ included in $\mathbf{K}$ :

$$
I_{\mathbf{K}}^{\varepsilon}=\bigcup_{Q_{i}^{\varepsilon} \subset \mathbf{K}} Q_{i}^{\varepsilon}, \quad Q_{i}^{\varepsilon}=\varepsilon(i+Q), i \in \mathbb{Z}^{d}
$$

Lemma 4.9. Consider the two linear forms $b$ and $\widetilde{b}_{h}$ respectively defined in (4.13) and (4.34). Under assumption (4.8), there exists a deterministic constant $C$ independent of $\eta, \varepsilon$ and $h$ such that

$$
\sup _{w_{h} \in \mathcal{V}_{h}} \frac{\left|\widetilde{b}_{h}\left(w_{h}\right)-b\left(w_{h}\right)\right|}{\left\|w_{h}\right\|_{H^{1}(\mathcal{D})}} \leq C \varepsilon\|f\|_{L^{2}(\mathcal{D})} .
$$

Before turning to the proof of Theorem 4.5, we first give some properties of the random variable $\lambda(\omega, h, \varepsilon)$ that appears in the right hand side of (4.39), and we next detail a two scale expansion of the highly oscillatory basis functions $\phi_{i}^{\varepsilon}$, which will be useful in the sequel.

Remark 4.10. We will show in Lemma 4.11 below that $\lambda$ defined by (4.40) is uniformly bounded with respect to $h, \varepsilon$ and $\omega$. Since $\mathcal{A}_{\eta}^{\star}$ is coercive, we deduce from (4.39) that $\widetilde{\mathcal{A}}_{\varepsilon, \eta}^{h}$ is also coercive, in the sense that there exists a deterministic constant $\alpha>0$, independent of $h, \varepsilon$ and $\eta$, such that

$$
\forall v_{h} \in \mathcal{V}_{h}, \quad \alpha\left\|v_{h}\right\|_{H^{1}(\mathcal{D})}^{2} \leq \widetilde{\mathcal{A}}_{\varepsilon, \eta}^{h}\left(v_{h}, v_{h}\right) .
$$

\subsubsection{Properties of $\lambda(\omega, h, \varepsilon)$}

We state here some useful properties of the random variable $\lambda(\omega, h, \varepsilon)$ that appears in (4.39). They will be proved in Appendix A. As mentioned above, we recall that the assumption $N_{\mathbf{K}} \geq \alpha(h / \varepsilon)^{d}$ that we make below is a regularity assumption on the macroscopic mesh (the volume of each element $\mathbf{K}$ is bounded from below by $\left.\alpha h^{d}\right)$.

Lemma 4.11. Let $\lambda(\omega, h, \varepsilon)$ be defined by (4.40). Then, there exists a deterministic constant $C$ such that, for any $h$ and $\varepsilon$, we have $0 \leq \lambda(\omega, h, \varepsilon) \leq C$ almost surely.

Assume now that the random matrix $A_{1}$ satisfies (4.7), where the law of $X_{k}(\omega)$ is absolutely continuous with respect to the Lebesgue measure. Assume furthermore that the number $N_{\mathbf{K}}=\operatorname{Card}\left(i ; Q_{i}^{\varepsilon} \subset \mathbf{K}\right)$ of cells in $\mathbf{K}$ satisfies $N_{\mathbf{K}} \geq \alpha\left(\frac{h}{\varepsilon}\right)^{d}$ for some $\alpha>0$ independent of the element $\mathbf{K}, h$ and $\varepsilon$. Then

$$
\mathbb{E}\left(\lambda(\cdot, h, \varepsilon)^{2}\right) \leq C \frac{\varepsilon^{d}}{h^{d}}[\ln (N(h))]^{2},
$$

where, we recall, $N(h)$ is the number of elements $\mathbf{K}$ in the domain $\mathcal{D}$ (which is of order $h^{-d}$ in dimension $d$ ) and $C$ is a deterministic constant independent of $h$ and $\varepsilon$. 
Because of the specific form (4.7) of $A_{1}$, we will see in the proof of that result (see Appendix A below) that

$$
\lambda(\omega, h, \varepsilon)=\max _{\mathbf{K}} \max _{1 \leq m, p \leq d}\left|S_{\mathbf{K}}^{m, p}\right|,
$$

where each random variable $S_{\mathbf{K}}^{m, p}$ is a normalized sum of $(h / \varepsilon)^{d}$ i.i.d. variables. Applying the Central Limit Theorem, we hence know that $S_{\mathbf{K}}^{m, p}$ converges, when $\varepsilon \rightarrow 0$, to a Gaussian random variable (up to an appropriate renormalization). Likewise, computing the expectation of $\left(S_{\mathbf{K}}^{m, p}\right)^{2}$ is not difficult. However, in the above lemma, the difficulty stems from the fact that $\lambda(\omega, h, \varepsilon)$ is the maximum of many such random variables $S_{\mathbf{K}}^{m, p}$ (in (4.43), the number of elements $\mathbf{K}$ is indeed of the order of $\left.h^{-d}\right)$. Our main task is hence to control how $\mathbb{E}\left(\lambda(\cdot, h, \varepsilon)^{2}\right)$ depends on $h$. See also Remark A.1.

\subsubsection{Two-scale expansion of the highly oscillatory basis functions}

Following [32], we recall here an expansion of $\phi_{i}^{\varepsilon, \mathbf{K}}$ that will be useful in the sequel. By definition (see (2.8) and (2.9)), we have, for any $1 \leq i \leq d+1$,

$$
\phi_{i}^{\varepsilon, \mathbf{K}}=\left.\sum_{j=1}^{d+1} \alpha_{i j} \chi_{j}^{\varepsilon, \mathbf{S}}\right|_{\mathbf{K}},
$$

where $\alpha_{i j}$ is such that

$$
\phi_{i}^{0, \mathbf{K}}=\left.\sum_{j=1}^{d+1} \alpha_{i j} \chi_{j}^{0, \mathbf{S}}\right|_{\mathbf{K}} .
$$

We thus first turn to $\chi_{i}^{\varepsilon, \mathbf{S}}$, which, by definition (see (2.7)), is the unique solution to

$$
-\operatorname{div}\left[A_{\text {per }}\left(\frac{x}{\varepsilon}\right) \nabla \chi_{i}^{\varepsilon, \mathbf{S}}(x)\right]=0 \text { in } \mathbf{S}, \quad \chi_{i}^{\varepsilon, \mathbf{S}}(x)=\chi_{i}^{0, \mathbf{S}}(x) \text { on } \partial \mathbf{S} .
$$

We introduce the function

$$
\theta_{i}^{\varepsilon, \mathbf{S}}(x)=\varepsilon^{-1}\left(\chi_{i}^{0, \mathbf{S}}(x)+\varepsilon \sum_{j=1}^{d} w_{e_{j}}^{0}\left(\frac{x}{\varepsilon}\right) \partial_{j} \chi_{i}^{0, \mathbf{S}}(x)-\chi_{i}^{\varepsilon, \mathbf{S}}(x)\right),
$$

where $w_{e_{i}}^{0}$ is solution to the periodic corrector problem (4.17). By construction, using (4.47), (4.17), (4.46) and the fact that $\nabla \chi_{i}^{0, \mathbf{S}}$ is constant on $\mathbf{S}$, we have

$$
\begin{aligned}
-\operatorname{div}\left[A_{\text {per }}\left(\frac{x}{\varepsilon}\right) \nabla \theta_{i}^{\varepsilon, \mathbf{S}}(x)\right] & =\frac{1}{\varepsilon} \operatorname{div}\left[A_{\text {per }}\left(\frac{x}{\varepsilon}\right) \nabla\left(\chi_{i}^{\varepsilon, \mathbf{S}}(x)-\chi_{i}^{0, \mathbf{S}}(x)-\varepsilon \sum_{j=1}^{d} w_{e_{j}}^{0}\left(\frac{x}{\varepsilon}\right) \partial_{j} \chi_{i}^{0, \mathbf{S}}\right)\right] \\
& =\frac{1}{\varepsilon} \operatorname{div}\left[A_{\text {per }}\left(\frac{x}{\varepsilon}\right) \nabla \chi_{i}^{\varepsilon, \mathbf{S}}(x)\right]-\frac{1}{\varepsilon} \sum_{j=1}^{d} \partial_{j} \chi_{i}^{0, \mathbf{S}} \operatorname{div}\left[A_{\mathrm{per}}\left(\frac{x}{\varepsilon}\right)\left(e_{j}+\nabla w_{e_{j}}^{0}\left(\frac{x}{\varepsilon}\right)\right)\right] \\
& =0,
\end{aligned}
$$

while, from (4.47), $\theta_{i}^{\varepsilon, \mathbf{S}}(x)=\sum_{j=1}^{d} \partial_{j} \chi_{i}^{0, \mathbf{S}}(x) w_{e_{j}}^{0}\left(\frac{x}{\varepsilon}\right)$ on $\partial \mathbf{S}$. So, by linearity, we obtain

$$
\theta_{i}^{\varepsilon, \mathbf{S}}(x)=\sum_{j=1}^{d} \partial_{j} \chi_{i}^{0, \mathbf{S}} \xi_{\varepsilon}^{j}(x)
$$


where $\xi_{\varepsilon}^{j} \in H^{1}(\mathbf{S})$ is the unique solution to

$$
-\operatorname{div}\left[A_{\text {per }}\left(\frac{x}{\varepsilon}\right) \nabla \xi_{\varepsilon}^{j}(x)\right]=0 \quad \text { in } \mathbf{S}, \quad \xi_{\varepsilon}^{j}(x)=w_{e_{j}}^{0}\left(\frac{x}{\varepsilon}\right) \quad \text { on } \partial \mathbf{S} .
$$

Using (4.47), we now obtain a useful relation between $\phi_{i}^{\varepsilon, \mathbf{K}}$ and $\phi_{i}^{0, \mathbf{K}}$. Indeed, collecting (4.44), (4.45), (4.47) and (4.48), we obtain the exact expression

$$
\phi_{i}^{\varepsilon, \mathbf{K}}(x)=\phi_{i}^{0, \mathbf{K}}(x)+\varepsilon \sum_{j=1}^{d}\left(w_{e_{j}}^{0}\left(\frac{x}{\varepsilon}\right)-\left.\xi_{\varepsilon}^{j}(x)\right|_{\mathbf{K}}\right) \partial_{j} \phi_{i}^{0, \mathbf{K}} .
$$

Recall now that $\phi_{i}^{\varepsilon, \mathbf{K}}(x)=\mathcal{R}_{\mathbf{K}}^{\varepsilon}\left(\phi_{i}^{0, \mathbf{K}}\right)$, by definition of the local operator $\mathcal{R}_{\mathbf{K}}^{\varepsilon}$ (see (4.35)). Correspondingly, the global operator $\mathcal{R}^{\varepsilon}$, defined on $\mathcal{V}_{h}$ by (4.36), equivalently writes

$$
\forall u \in \mathcal{V}_{h}, \quad \mathcal{R}^{\varepsilon}(u)=u+\varepsilon \sum_{j=1}^{d}\left(w_{e_{j}}^{0}\left(\frac{\dot{\varepsilon}}{\varepsilon}\right)-\overline{\xi_{\varepsilon}^{j}}\right) \partial_{j} u,
$$

where $\overline{\xi_{\varepsilon}^{j}}$ is locally defined on each element $\mathbf{K}$ by $\left.\overline{\xi_{\varepsilon}^{j}}\right|_{\mathbf{K}}=\left.\xi_{\varepsilon}^{j}\right|_{\mathbf{K}}$. By construction, for each $\mathbf{K}, \overline{\xi_{\varepsilon}^{j}} \in H^{1}(\mathbf{K})$, but it a priori does not belong to $H^{1}(\mathcal{D})$. The relation (4.51) allows to extend the operator $\mathcal{R}^{\varepsilon}$ on $H^{1}(\mathcal{D})$.

We now recall the following bound on the function $\xi_{\varepsilon}^{j}$, that appears in (4.50). In [32], this lemma is stated in dimension $d=2$, but its proof, which essentially makes use Lemma 16 of [9], carries over to any dimension.

Lemma 4.12 (see [32], Lem. 2.1). Let $\xi_{\varepsilon}^{j}$ be the solution to (4.49), with $A_{\text {per }}$ satisfying (4.8). Consider $\mathbf{K} \subset \mathbf{S}$, with $\operatorname{diam}(\mathbf{K})=h$ and $\operatorname{dist}(\mathbf{K}, \partial \mathbf{S}) \geq h$. Then there exists a constant $C$ independent of $h$ and $\varepsilon$ such that

$$
\left\|\nabla \xi_{\varepsilon}^{j}\right\|_{L^{\infty}(\mathbf{K})} \leq \frac{C}{h}
$$

\subsubsection{Proof of Theorem 4.5}

The proof is based on the bound (4.38) in Lemma 4.7, where the bilinear form $\mathcal{A}_{\varepsilon, \eta}^{h}$ and the linear form $b$ are defined by (4.33). In Step 1, we show that the bilinear form $\mathcal{A}_{\varepsilon, \eta}^{h}$ is coercive for the norm $\|\cdot\|_{H_{h}^{1}}$ defined by (3.2). Step 2 is devoted to appropriately selecting an element $v_{h} \in \mathcal{W}_{h}$ such that $\left\|u_{\eta}^{\varepsilon}-v_{h}\right\|_{H_{h}^{1}}$ can be analytically estimated. This will provide a bound on the first term in the right hand side of (4.38). In Step 3, we bound from above the second term in the right hand side of (4.38), using Lemmas 4.8 and 4.9. Step 4 collects our estimates and concludes.

Step 1. We first show that the bilinear form $\mathcal{A}_{\varepsilon, \eta}^{h}$ defined by (4.33) is coercive for the norm $\|\cdot\|_{H_{h}^{1}}$ defined by (3.2). Consider the bilinear form $\widetilde{\mathcal{A}}_{\varepsilon, \eta}^{h}$ defined by (4.34). We pointed out above (see Rem. 4.10) that it is coercive on $\mathcal{V}_{h}$. Hence, there exists $\alpha>0$ such that, for all $v_{h} \in \mathcal{W}_{h}$,

$$
\alpha\left\|\widetilde{v}_{h}\right\|_{H^{1}(\mathcal{D})}^{2} \leq \widetilde{\mathcal{A}}_{\varepsilon, \eta}^{h}\left(\widetilde{v}_{h}, \widetilde{v}_{h}\right)=\mathcal{A}_{\varepsilon, \eta}^{h}\left(v_{h}, v_{h}\right),
$$

where $\widetilde{v}_{h} \in \mathcal{V}_{h}$ is such that $v_{h}=\mathcal{R}^{\varepsilon}\left(\widetilde{v}_{h}\right)$. Since, in the bilinear form $\mathcal{A}_{\varepsilon, \eta}^{h}$, the matrix $A_{\eta}$ is bounded, we deduce the estimate

$$
\left\|\widetilde{v}_{h}\right\|_{H^{1}(\mathcal{D})}^{2} \leq C\left\|v_{h}\right\|_{H_{h}^{1}}^{2},
$$

that we will use in the sequel. The sequel of this step is devoted to proving that there exists $\widetilde{C}$ independent of $h$ and $\varepsilon$ such that, for all $\widetilde{v}_{h} \in \mathcal{V}_{h}$,

$$
\left\|v_{h}\right\|_{H_{h}^{1}}^{2} \leq \widetilde{C}\left\|\widetilde{v}_{h}\right\|_{H^{1}(\mathcal{D})}^{2} \quad \text { with } v_{h}=\mathcal{R}^{\varepsilon}\left(\widetilde{v}_{h}\right) .
$$

Combined with (4.53), this shows that $\mathcal{A}_{\varepsilon, \eta}^{h}$ is coercive for the norm $\|\cdot\|_{H_{h}^{1}}$. 
To prove (4.55), we first write that, since $v_{h}=\mathcal{R}^{\varepsilon}\left(\widetilde{v}_{h}\right)$ and $\widetilde{v}_{h} \in \mathcal{V}_{h}$, there exist some coefficients $\left\{\beta_{i}\right\}_{i=1}^{L}$ such that, for any $x \in \mathcal{D}, \widetilde{v}_{h}=\sum_{i=1}^{L} \beta_{i} \phi_{i}^{0}$ and $v_{h}=\mathcal{R}^{\varepsilon}\left(\widetilde{v}_{h}\right)=\sum_{i=1}^{L} \beta_{i} \phi_{i}^{\varepsilon}$. Consider now an element $\mathbf{K}$, and its corresponding oversampling domain $\mathbf{S}$. We know from (2.8) and (2.10) that

$$
\forall x \in \mathbf{K}, \quad \widetilde{v}_{h}(x)=\sum_{i=1}^{L} \sum_{j=1}^{d+1} \beta_{i} \alpha_{i j} \chi_{j}^{0, \mathbf{S}}(x), \quad v_{h}(x)=\sum_{i=1}^{L} \sum_{j=1}^{d+1} \beta_{i} \alpha_{i j} \chi_{j}^{\varepsilon, \mathbf{S}}(x) .
$$

Consider now the functions

$$
\widetilde{w}_{h}^{\mathbf{S}}(x):=\sum_{i=1}^{L} \sum_{j=1}^{d+1} \beta_{i} \alpha_{i j} \chi_{j}^{0, \mathbf{S}}(x), \quad w_{h}^{\mathbf{S}}(x):=\sum_{i=1}^{L} \sum_{j=1}^{d+1} \beta_{i} \alpha_{i j} \chi_{j}^{\varepsilon, \mathbf{S}}(x),
$$

defined on $\mathbf{S}$, and that satisfy, by construction,

$$
\forall x \in \mathbf{K}, \quad \widetilde{v}_{h}(x)=\widetilde{w}_{h}^{\mathbf{S}}(x), \quad v_{h}(x)=w_{h}^{\mathbf{S}}(x) .
$$

In view of (2.7), we have

$$
-\operatorname{div}\left[A^{\varepsilon}(x) \nabla w_{h}^{\mathbf{S}}(x)\right]=0 \quad \text { in } \mathbf{S}, \quad w_{h}^{\mathbf{S}}=\widetilde{w}_{h}^{\mathbf{S}} \text { on } \partial \mathbf{S},
$$

which implies that

$$
\left\|w_{h}^{\mathbf{S}}\right\|_{H^{1}(\mathbf{S})} \leq C\left\|\widetilde{w}_{h}^{\mathbf{S}}\right\|_{H^{1}(\mathbf{S})} .
$$

We deduce from (4.56) and the above bound that

$$
\left\|v_{h}\right\|_{H^{1}(\mathbf{K})}=\left\|w_{h}^{\mathbf{S}}\right\|_{H^{1}(\mathbf{K})} \leq\left\|w_{h}^{\mathbf{S}}\right\|_{H^{1}(\mathbf{S})} \leq C\left\|\widetilde{w}_{h}^{\mathbf{S}}\right\|_{H^{1}(\mathbf{S})} .
$$

We next see that there exists $C$ independent of $h$ such that, for any piecewise-affine function $\tau$ on $\mathbf{S}$, we have $\|\tau\|_{H^{1}(\mathbf{S})} \leq C\|\tau\|_{H^{1}(\mathbf{K})}$, provided there exists $0<c_{-} \leq c_{+}$independent of the element such that $c_{-} \leq \frac{|\mathbf{S}|}{|\mathbf{K}|} \leq c_{+}$. Using this bound for $\tau=\widetilde{w}_{h}^{\mathbf{S}}$, we infer from (4.57) and (4.56) that

$$
\left\|v_{h}\right\|_{H^{1}(\mathbf{K})} \leq C\left\|\widetilde{w}_{h}^{\mathbf{S}}\right\|_{H^{1}(\mathbf{S})} \leq \bar{C}\left\|\widetilde{w}_{h}^{\mathbf{S}}\right\|_{H^{1}(\mathbf{K})}=\bar{C}\left\|\widetilde{v}_{h}\right\|_{H^{1}(\mathbf{K})}
$$

Summing over all elements $\mathbf{K}$, we obtain (4.55), and this concludes this first step.

Step 2. We now bound the first term of the right-hand side of (4.38), which is the best approximation error. Let $\Pi^{h} u_{\eta}^{\star}$ be the $H^{1}$ projection of $u_{\eta}^{\star}$, solution to (4.10), on the standard FEM space $\mathcal{V}_{h}$. We have $\mathcal{R}^{\varepsilon}\left(\Pi^{h} u_{\eta}^{\star}\right) \in \mathcal{W}_{h}$ (recall $\mathcal{R}^{\varepsilon}$ is defined by (4.36), and equivalently writes as in (4.51)). Our argument is based on the following triangle inequality:

$$
\begin{aligned}
\mathbb{E}\left[\inf _{v_{h} \in \mathcal{W}_{h}}\left\|u_{\eta}^{\varepsilon}-v_{h}\right\|_{H_{h}^{1}}^{2}\right] \leq & 2 \mathbb{E}\left[\left\|u_{\eta}^{\varepsilon}-v_{\eta}^{\varepsilon}\right\|_{H^{1}(\mathcal{D})}^{2}\right]+2 \mathbb{E}\left[\inf _{v_{h} \in \mathcal{W}_{h}}\left\|v_{\eta}^{\varepsilon}-v_{h}\right\|_{H_{h}^{1}}^{2}\right] \\
\leq & 2 \mathbb{E}\left[\left\|u_{\eta}^{\varepsilon}-v_{\eta}^{\varepsilon}\right\|_{H^{1}(\mathcal{D})}^{2}\right]+2 \mathbb{E}\left[\left\|v_{\eta}^{\varepsilon}-\mathcal{R}^{\varepsilon}\left(\Pi^{h} u_{\eta}^{\star}\right)\right\|_{H_{h}^{1}}^{2}\right] \\
\leq & 2 \mathbb{E}\left[\left\|u_{\eta}^{\varepsilon}-v_{\eta}^{\varepsilon}\right\|_{H^{1}(\mathcal{D})}^{2}\right]+4 \mathbb{E}\left[\left\|v_{\eta}^{\varepsilon}-\mathcal{R}^{\varepsilon}\left(u_{\eta}^{\star}\right)\right\|_{H_{h}^{1}}^{2}\right] \\
& +4\left\|\mathcal{R}^{\varepsilon}\left(u_{\eta}^{\star}\right)-\mathcal{R}^{\varepsilon}\left(\Pi^{h} u_{\eta}^{\star}\right)\right\|_{H_{h}^{1}}^{2}
\end{aligned}
$$

where $v_{\eta}^{\varepsilon}(\cdot, \omega) \in H^{1}(\mathcal{D})$ is defined by $(4.25)$. The estimate (4.28) in Theorem 4.2 bounds the first term from above. In the following two sub-steps, we bound the other two terms of (4.60). 
Step 2a. Bound on $\mathbb{E}\left(\left\|v_{\eta}^{\varepsilon}-\mathcal{R}^{\varepsilon}\left(u_{\eta}^{\star}\right)\right\|_{H_{h}^{1}}^{2}\right)$

Using the expansion (4.19) of $u_{\eta}^{\star}$ in a series in powers of $\eta$, and (4.51), we write

$$
\mathcal{R}^{\varepsilon}\left(u_{\eta}^{\star}\right)=u_{0}^{\star}+\eta \mathbb{E}\left(X_{0}\right) \bar{u}_{1}^{\star}+\varepsilon \sum_{p=1}^{d}\left(w_{e_{p}}^{0}\left(\frac{\dot{\varepsilon}}{\varepsilon}\right)-\overline{\xi_{\varepsilon}^{p}}\right)\left(\partial_{p} u_{0}^{\star}+\eta \mathbb{E}\left(X_{0}\right) \partial_{p} \bar{u}_{1}^{\star}\right)+\eta^{2} g_{\eta},
$$

where

$$
g_{\eta}=r_{\eta}+\varepsilon \sum_{p=1}^{d}\left(w_{e_{p}}^{0}\left(\frac{\dot{\varepsilon}}{\varepsilon}\right)-\overline{\xi_{\varepsilon}^{p}}\right) \partial_{p} r_{\eta}
$$

Using (4.25), we thus have

$$
\begin{aligned}
v_{\eta}^{\varepsilon}(\cdot, \omega)-\mathcal{R}^{\varepsilon}\left(u_{\eta}^{\star}\right)= & \eta \varepsilon \sum_{p=1}^{d}\left(\mathbb{E}\left(X_{0}\right) \psi_{e_{p}}\left(\frac{\dot{\varepsilon}}{\varepsilon}\right) \partial_{p} u_{0}^{\star}+\sum_{k \in I_{\varepsilon}}\left(X_{k}(\omega)-\mathbb{E}\left(X_{0}\right)\right) \chi_{e_{p}}\left(\frac{\dot{\varepsilon}}{\varepsilon}-k\right) \partial_{p} u_{0}^{\star}\right) \\
& +\varepsilon \sum_{p=1}^{d} \overline{\xi_{\varepsilon}^{p}}\left(\partial_{p} u_{\eta}^{\star}-\eta^{2} \partial_{p} r_{\eta}\right)-\eta^{2} g_{\eta} .
\end{aligned}
$$

To bound $\mathbb{E}\left[\sum_{\mathbf{K}}\left\|v_{\eta}^{\varepsilon}-\mathcal{R}^{\varepsilon}\left(u_{\eta}^{\star}\right)\right\|_{H^{1}(\mathbf{K})}^{2}\right]$, we first establish a few simple results. First, there exists $\delta>0$ such that, for any $1 \leq p \leq d$, we have

$$
w_{e_{p}}^{0} \in C^{1, \delta}(Q),
$$

where $w_{p}^{0}$ is the periodic corrector, solution to (4.17). This is a consequence of the fact that $A_{\text {per }}$ is Höldercontinuous (see Assumption (4.8)), in view of Theorem 8.22 and Corollary 8.36 of [34]. We infer from (4.63) and the periodicity of $w_{e_{p}}^{0}$ that, for any $1 \leq p \leq d$, we have

$$
w_{e_{p}}^{0} \in W^{1, \infty}\left(\mathbb{R}^{d}\right) .
$$

Second, for any $1 \leq p \leq d$, we have

$$
\left\|\chi_{e_{p}}\left(\frac{\dot{\varepsilon}}{\varepsilon}-k\right)\right\|_{L^{2}(\mathcal{D})}^{2} \leq C \varepsilon^{d} R_{d, \varepsilon} \quad \text { and } \quad \nabla \chi_{e_{p}} \in\left(L^{2}\left(\mathbb{R}^{d}\right)\right)^{d},
$$

where $C$ is independent from $\varepsilon$ and $k, R_{d, \varepsilon}=1$ if $d>2$ and $R_{d, \varepsilon}=1+\ln (1 / \varepsilon)$ if $d=2$ (see (4.27) and (4.31)). Third, we see that, for any $1 \leq p \leq d$,

$$
\left\|\overline{\xi_{\varepsilon}^{p}}\right\|_{L^{\infty}(\mathcal{D})} \leq C \quad \text { and } \quad\left\|\nabla \xi_{\varepsilon}^{p}\right\|_{L^{\infty}(\mathbf{K})} \leq \frac{C}{h},
$$

where $C$ is independent from $\varepsilon$ and $h$. The second assertion is given by Lemma 4.12 above, whereas the first assertion comes from (4.49): using again Theorem 8.22 and Corollary 8.36 of [34], and (4.63), we first see that, for any $\mathbf{S}, \xi_{\varepsilon}^{p} \in C^{1, \delta}(\mathbf{S})$ for some $\delta>0$. Using next the maximum principle on (4.49), we have $\left\|\xi_{\varepsilon}^{p}\right\|_{L^{\infty}(\mathbf{S})} \leq\left\|w_{e_{p}}\left(\frac{\cdot}{\varepsilon}\right)\right\|_{L^{\infty}\left(\mathbb{R}^{d}\right)} \leq C$. Lastly, using (4.61), (4.23), (4.64) and (4.66), we obtain that, for any element $\mathbf{K}$,

$$
\left\|g_{\eta}\right\|_{H^{1}(\mathbf{K})} \leq C\left\|r_{\eta}\right\|_{H^{2}(\mathbf{K})}\left(1+\varepsilon+\frac{\varepsilon}{h}\right) \leq C\left\|r_{\eta}\right\|_{H^{2}(\mathbf{K})},
$$

hence

$$
\left\|g_{\eta}\right\|_{L^{2}(\mathcal{D})}^{2} \leq \sum_{\mathbf{K}}\left\|g_{\eta}\right\|_{H^{1}(\mathbf{K})}^{2} \leq C\left\|r_{\eta}\right\|_{H^{2}(\mathcal{D})}^{2} \leq C .
$$


We are now in position to estimate (4.62). Using that $u_{0}^{\star} \in W^{1, \infty}(\mathcal{D})$, we deduce from $(4.65),(4.66)$ and $(4.67)$ that

$$
\begin{aligned}
\mathbb{E}\left[\left\|v_{\eta}^{\varepsilon}-\mathcal{R}^{\varepsilon}\left(u_{\eta}^{\star}\right)\right\|_{L^{2}(\mathcal{D})}^{2}\right] \leq & C \eta^{2} \varepsilon^{2} \sum_{p=1}^{d}\left\|\partial_{p} u_{0}^{\star}\right\|_{L^{\infty}}^{2}\left[\mathbb{E}\left(X_{0}\right)^{2}\left\|\psi_{e_{p}}\left(\frac{\cdot}{\varepsilon}\right)\right\|_{L^{2}(\mathcal{D})}^{2}\right. \\
& \left.+\operatorname{Var}\left(X_{0}\right) \sum_{k \in I_{\varepsilon}}\left\|\chi_{e_{p}}\left(\frac{\dot{-}}{\varepsilon}-k\right)\right\|_{L^{2}(\mathcal{D})}^{2}\right] \\
& +C \varepsilon^{2} \sum_{p=1}^{d}\left\|\bar{\xi}_{\bar{\varepsilon}}^{p}\right\|_{L^{\infty}(\mathcal{D})}^{2}\left(\left\|\nabla u_{\eta}^{\star}\right\|_{L^{2}(\mathcal{D})}^{2}+\eta^{4}\left\|\nabla r_{\eta}\right\|_{L^{2}(\mathcal{D})}^{2}\right)+C \eta^{4}\left\|g_{\eta}\right\|_{L^{2}(\mathcal{D})}^{2} \\
\leq & C \eta^{2} \varepsilon^{2}\left(1+\left(\operatorname{Card} I_{\varepsilon}\right) \varepsilon^{d} R_{d, \varepsilon}\right)+C \varepsilon^{2}+C \eta^{4} \\
\leq & C\left[\eta^{2} \varepsilon^{2} R_{d, \varepsilon}+\varepsilon^{2}+\eta^{4}\right]
\end{aligned}
$$

for some constant $C$ independent of $\varepsilon, \eta$ and $h$, and where, we recall, $R_{d, \varepsilon}=1+\ln (1 / \varepsilon)$ if $d=2$ and $R_{d, \varepsilon}=1$ if $d>2$.

We thus have a bound on $v_{\eta}^{\varepsilon}(\cdot, \omega)-\mathcal{R}^{\varepsilon}\left(u_{\eta}^{\star}\right)$ in the $L^{2}$ norm. To prove a bound in the broken $H^{1}$ norm, we consider $\nabla v_{\eta}^{\varepsilon}(\cdot, \omega)-\nabla \mathcal{R}^{\varepsilon}\left(u_{\eta}^{\star}\right)$ : we see from (4.62) that, in each element $\mathbf{K}$,

$$
\nabla v_{\eta}^{\varepsilon}(\cdot, \omega)-\nabla \mathcal{R}^{\varepsilon}\left(u_{\eta}^{\star}\right)=\eta \varepsilon D_{0}+\eta D_{1}+D_{2}-\eta^{2} \nabla g_{\eta}
$$

where

$$
\begin{aligned}
& D_{0}=\sum_{p=1}^{d}\left(\mathbb{E}\left(X_{0}\right) \psi_{e_{p}}\left(\frac{\dot{z}}{\varepsilon}\right) \nabla \partial_{p} u_{0}^{\star}+\sum_{k \in I_{\varepsilon}}\left(X_{k}(\omega)-\mathbb{E}\left(X_{0}\right)\right) \chi_{e_{p}}\left(\frac{\dot{-}}{\varepsilon}-k\right) \nabla \partial_{p} u_{0}^{\star}\right), \\
& D_{1}=\sum_{p=1}^{d}\left(\mathbb{E}\left(X_{0}\right) \nabla \psi_{e_{p}}\left(\frac{\dot{\varepsilon}}{\varepsilon}\right) \partial_{p} u_{0}^{\star}+\sum_{k \in I_{\varepsilon}}\left(X_{k}(\omega)-\mathbb{E}\left(X_{0}\right)\right) \nabla \chi_{e_{p}}\left(\frac{\dot{\bar{\varepsilon}}}{\varepsilon}-k\right) \partial_{p} u_{0}^{\star}\right), \\
& D_{2}=\varepsilon \sum_{p=1}^{d}\left(\overline{\xi_{\varepsilon}^{p}} \nabla \partial_{p}\left(u_{\eta}^{\star}-\eta^{2} r_{\eta}\right)+\nabla \overline{\xi_{\varepsilon}^{p}} \partial_{p}\left(u_{\eta}^{\star}-\eta^{2} r_{\eta}\right)\right) .
\end{aligned}
$$

Note that $D_{0}$ and $D_{1}$ are globally defined on $\mathcal{D}$, but $D_{2}$ is not (as $\overline{\xi_{\varepsilon}^{p}}$ may have jumps from one element $\mathbf{K}$ to the other). We now bound these three quantities. Using (4.65) and the fact that $u_{0}^{\star} \in W^{2, \infty}(\mathcal{D})$, we have

$$
\begin{aligned}
\mathbb{E}\left(\left\|D_{0}\right\|_{L^{2}(\mathcal{D})}^{2}\right) & \leq C \sum_{p=1}^{d}\left\|\nabla \partial_{p} u_{0}^{\star}\right\|_{L^{\infty}}^{2}\left(\mathbb{E}\left(X_{0}\right)^{2}\left\|\psi_{e_{p}}\left(\frac{\dot{ }}{\varepsilon}\right)\right\|_{L^{2}(\mathcal{D})}^{2}+\operatorname{Var}\left(X_{0}\right) \sum_{k \in I_{\varepsilon}}\left\|\chi_{e_{p}}\left(\frac{\dot{\bar{\varepsilon}}}{\varepsilon}-k\right)\right\|_{L^{2}(\mathcal{D})}^{2}\right) \\
& \leq C R_{d, \varepsilon},
\end{aligned}
$$

where $C$ is a constant independent of $\varepsilon$ and $h$. We now turn to $D_{1}$ : using again $(4.65)$ and that $u_{0}^{\star} \in W^{1, \infty}(\mathcal{D})$, we obtain

$$
\begin{aligned}
\mathbb{E}\left(\left\|D_{1}\right\|_{L^{2}(\mathcal{D})}^{2}\right) & \leq C \sum_{p=1}^{d}\left\|\partial_{p} u_{0}^{\star}\right\|_{L^{\infty}}^{2}\left(\mathbb{E}\left(X_{0}\right)^{2}\left\|\nabla \psi_{e_{p}}\left(\frac{\dot{\bar{\varepsilon}}}{\varepsilon}\right)\right\|_{L^{2}(\mathcal{D})}^{2}+\operatorname{Var}\left(X_{0}\right) \sum_{k \in I_{\varepsilon}}\left\|\nabla \chi_{e_{p}}\left(\frac{\dot{\bar{\varepsilon}}}{\varepsilon}-k\right)\right\|_{L^{2}(\mathcal{D})}^{2}\right) \\
& \leq C \sum_{p=1}^{d}\left(1+\operatorname{Var}\left(X_{0}\right) \sum_{k \in I_{\varepsilon}} \varepsilon^{d}\left\|\nabla \chi_{e_{p}}\right\|_{L^{2}\left(\mathbb{R}^{d}\right)}^{2}\right) \\
& \leq C,
\end{aligned}
$$


where $C$ is a constant independent of $\varepsilon$ and $h$. Turning to $D_{2}$, using (4.66), we have in each element $\mathbf{K}$ that

$$
\left\|D_{2}\right\|_{L^{2}(\mathbf{K})}^{2} \leq C \varepsilon^{2}\left\|u_{\eta}^{\star}-\eta^{2} r_{\eta}\right\|_{H^{2}(\mathbf{K})}^{2}\left(1+\frac{1}{h^{2}}\right) \leq C \frac{\varepsilon^{2}}{h^{2}}\left\|u_{\eta}^{\star}-\eta^{2} r_{\eta}\right\|_{H^{2}(\mathbf{K})}^{2},
$$

hence, using (4.23),

$$
\sum_{\mathbf{K}}\left\|D_{2}\right\|_{L^{2}(\mathbf{K})}^{2} \leq C \frac{\varepsilon^{2}}{h^{2}}\left\|u_{\eta}^{\star}-\eta^{2} r_{\eta}\right\|_{H^{2}(\mathcal{D})}^{2} \leq C \frac{\varepsilon^{2}}{h^{2}} .
$$

Collecting (4.69), (4.70), (4.71), (4.72) and (4.67), we obtain that

$$
\mathbb{E}\left[\sum_{\mathbf{K}}\left\|\nabla v_{\eta}^{\varepsilon}-\nabla \mathcal{R}^{\varepsilon}\left(u_{\eta}^{\star}\right)\right\|_{L^{2}(\mathbf{K})}^{2}\right] \leq C\left(\eta^{2} \varepsilon^{2} R_{d, \varepsilon}+\eta^{2}+\frac{\varepsilon^{2}}{h^{2}}+\eta^{4}\right) .
$$

Collecting this bound with (4.68), and assuming that $|\eta|<1$ and $\varepsilon^{2} R_{d, \varepsilon} \leq 1$, we deduce that

$$
\mathbb{E}\left[\left\|v_{\eta}^{\varepsilon}-\mathcal{R}^{\varepsilon}\left(u_{\eta}^{\star}\right)\right\|_{H_{h}^{1}}^{2}\right]=\mathbb{E}\left[\sum_{\mathbf{K}}\left\|v_{\eta}^{\varepsilon}-\mathcal{R}^{\varepsilon}\left(u_{\eta}^{\star}\right)\right\|_{H^{1}(\mathbf{K})}^{2}\right] \leq C\left(\eta^{2}+\frac{\varepsilon^{2}}{h^{2}}\right),
$$

where $C$ is independent from $\varepsilon, h$ and $\eta$.

Step 2b. Bound on $\left\|\mathcal{R}^{\varepsilon}\left(u_{\eta}^{\star}\right)-\mathcal{R}^{\varepsilon}\left(\Pi^{h} u_{\eta}^{\star}\right)\right\|_{H_{h}^{1}}^{2}$

Recall that $\Pi^{h} u_{\eta}^{\star}$ is the $H^{1}$ projection of $u_{\eta}^{\star}$ (on the standard $\mathbb{P}_{1}$ FEM space $\mathcal{V}_{h}$ ), hence $\left\|\Pi^{h} u_{\eta}^{\star}\right\|_{H^{1}(\mathcal{D})} \leq$ $\left\|u_{\eta}^{\star}\right\|_{H^{1}(\mathcal{D})}$. In addition, using (4.23), we have, using a standard result from the theory of $\mathbb{P}_{1}$ finite elements (see [22], Thm. 3.1.6 p. 124)

$$
\left\|u_{\eta}^{\star}-\Pi^{h} u_{\eta}^{\star}\right\|_{L^{2}(\mathcal{D})}+h\left\|u_{\eta}^{\star}-\Pi^{h} u_{\eta}^{\star}\right\|_{H^{1}(\mathcal{D})} \leq C h^{2}\left\|\nabla^{2} u_{\eta}^{\star}\right\|_{L^{2}(\mathcal{D})} \leq C h^{2},
$$

where $C$ is a constant independent of $h$ and $\eta$. In view of (4.51), we have

$$
\mathcal{R}^{\varepsilon}\left(u_{\eta}^{\star}\right)-\mathcal{R}^{\varepsilon}\left(\Pi^{h} u_{\eta}^{\star}\right)=u_{\eta}^{\star}-\Pi^{h} u_{\eta}^{\star}+\varepsilon \sum_{p=1}^{d}\left(w_{e_{p}}^{0}\left(\frac{\dot{\varepsilon}}{\varepsilon}\right)-\overline{\xi_{\varepsilon}^{p}}\right) \partial_{p}\left(u_{\eta}^{\star}-\Pi^{h} u_{\eta}^{\star}\right) .
$$

We deduce from (4.64), (4.66) and (4.74) that

$$
\left\|\mathcal{R}^{\varepsilon}\left(u_{\eta}^{\star}\right)-\mathcal{R}^{\varepsilon}\left(\Pi^{h} u_{\eta}^{\star}\right)\right\|_{L^{2}(\mathcal{D})} \leq C\left(h^{2}+\varepsilon h\right) .
$$

We now turn to bounding the gradients. Recall that $\nabla\left(\Pi^{h} u_{\eta}^{\star}\right)$ is constant in each element $\mathbf{K}$. We thus have, using (4.64) and (4.66), that

$$
\begin{aligned}
\left\|\nabla \mathcal{R}^{\varepsilon}\left(u_{\eta}^{\star}\right)-\nabla \mathcal{R}^{\varepsilon}\left(\Pi^{h} u_{\eta}^{\star}\right)\right\|_{L^{2}(\mathbf{K}) \leq} & \left\|u_{\eta}^{\star}-\Pi^{h} u_{\eta}^{\star}\right\|_{H^{1}(\mathbf{K})}\left(1+\sum_{p=1}^{d}\left(\left\|\nabla w_{e_{p}}^{0}\left(\frac{\dot{\varepsilon}}{\varepsilon}\right)\right\|_{L^{\infty}(\mathbf{K})}+\varepsilon\left\|\nabla \xi_{\varepsilon}^{p}\right\|_{L^{\infty}(\mathbf{K})}\right)\right) \\
& +\varepsilon \sum_{p=1}^{d}\left\|w_{e_{p}}^{0}\left(\frac{\dot{\varepsilon}}{\varepsilon}\right)-\xi_{\varepsilon}^{p}\right\|_{L^{\infty}(\mathbf{K})}\left\|u_{\eta}^{\star}\right\|_{H^{2}(\mathbf{K})} \\
\leq & C\left\|u_{\eta}^{\star}-\Pi^{h} u_{\eta}^{\star}\right\|_{H^{1}(\mathbf{K})}\left(1+\frac{\varepsilon}{h}\right)+\varepsilon\left\|u_{\eta}^{\star}\right\|_{H^{2}(\mathbf{K})} .
\end{aligned}
$$

We then deduce, using (4.74) and (4.23), that

$$
\begin{aligned}
\sum_{\mathbf{K}}\left\|\nabla \mathcal{R}^{\varepsilon}\left(u_{\eta}^{\star}\right)-\nabla \mathcal{R}^{\varepsilon}\left(\Pi^{h} u_{\eta}^{\star}\right)\right\|_{L^{2}(\mathbf{K})}^{2} & \leq C\left\|u_{\eta}^{\star}-\Pi^{h} u_{\eta}^{\star}\right\|_{H^{1}(\mathcal{D})}^{2}\left(1+\frac{\varepsilon}{h}\right)^{2}+\varepsilon^{2}\left\|u_{\eta}^{\star}\right\|_{H^{2}(\mathcal{D})}^{2} \\
& \leq C h^{2}\left(1+\frac{\varepsilon}{h}\right)^{2}+C \varepsilon^{2}
\end{aligned}
$$


where $C$ is a constant independent of $\varepsilon, \eta$ and $h$. Collecting this bound and (4.75), we obtain

$$
\left\|\mathcal{R}^{\varepsilon}\left(\Pi^{h} u_{\eta}^{\star}\right)-\mathcal{R}^{\varepsilon}\left(u_{\eta}^{\star}\right)\right\|_{H_{h}^{1}}^{2}=\sum_{\mathbf{K}}\left\|\mathcal{R}^{\varepsilon}\left(u_{\eta}^{\star}\right)-\mathcal{R}^{\varepsilon}\left(\Pi^{h} u_{\eta}^{\star}\right)\right\|_{H^{1}(\mathbf{K})}^{2} \leq C\left(h^{2}+\varepsilon^{2}\right),
$$

where $C$ is a constant independent of $\varepsilon, \eta$ and $h$.

Step 2c. We are now in position to bound the first term in (4.38). We infer from (4.60), (4.28), (4.73) and (4.76) that

$$
\sqrt{\mathbb{E}\left(\inf _{v_{h} \in \mathcal{W}_{h}}\left\|u_{\eta}^{\varepsilon}-v_{h}\right\|_{H_{h}^{1}}^{2}\right)} \leq C\left(\sqrt{\varepsilon}+\eta \sqrt{\varepsilon \ln (1 / \varepsilon)}+\eta+\frac{\varepsilon}{h}+h\right) \leq C\left(\sqrt{\varepsilon}+\eta+\frac{\varepsilon}{h}+h\right),
$$

where we have assumed that $\varepsilon \ln (1 / \varepsilon) \leq 1$.

Step 3. We next turn to estimating the non-conforming error, namely the second term of the right-hand side of (4.38). For any $w_{h} \in \mathcal{W}_{h}$, introduce $\widetilde{w}_{h} \in \mathcal{V}_{h}$ such that $\mathcal{R}^{\varepsilon}\left(\widetilde{w}_{h}\right)=w_{h}$ (recall that $\mathcal{R}^{\varepsilon}$ is defined by (4.36)). We note that $b\left(w_{h}\right)=\widetilde{b}_{h}\left(\widetilde{w}_{h}\right)$, where the linear forms $b$ and $\widetilde{b}_{h}$ are defined by (4.33) and (4.34). Using the weak form of the homogenized equation (see (4.13)), we see that $b\left(\widetilde{w}_{h}\right)=\mathcal{A}_{\eta}^{\star}\left(u_{\eta}^{\star}, \widetilde{w}_{h}\right)$. In addition, by definition of $\widetilde{\mathcal{A}}_{\varepsilon, \eta}^{h}$ (see (4.34)), we have $\widetilde{\mathcal{A}}_{\varepsilon, \eta}^{h}\left(\Pi^{h} u_{\eta}^{\star}, \widetilde{w}_{h}\right)=\mathcal{A}_{\varepsilon, \eta}^{h}\left(\mathcal{R}^{\varepsilon}\left(\Pi^{h} u_{\eta}^{\star}\right), w_{h}\right)$. For any $w_{h} \in \mathcal{W}_{h}$, we have

$$
\begin{aligned}
& \left|\mathcal{A}_{\varepsilon, \eta}^{h}\left(u_{\eta}^{\varepsilon}, w_{h}\right)-b\left(w_{h}\right)\right| \\
\leq & \left|\mathcal{A}_{\varepsilon, \eta}^{h}\left(u_{\eta}^{\varepsilon}, w_{h}\right)-\mathcal{A}_{\varepsilon, \eta}^{h}\left(\mathcal{R}^{\varepsilon}\left(\Pi^{h} u_{\eta}^{\star}\right), w_{h}\right)\right|+\left|\mathcal{A}_{\varepsilon, \eta}^{h}\left(\mathcal{R}^{\varepsilon}\left(\Pi^{h} u_{\eta}^{\star}\right), w_{h}\right)-b\left(\widetilde{w}_{h}\right)\right|+\left|b\left(\widetilde{w}_{h}\right)-b\left(w_{h}\right)\right| \\
\leq & \left\|A_{\eta}\right\|_{L^{\infty}}\left\|u_{\eta}^{\varepsilon}-\mathcal{R}^{\varepsilon}\left(\Pi^{h} u_{\eta}^{\star}\right)\right\|_{H_{h}^{1}}\left\|w_{h}\right\|_{H_{h}^{1}}+\left|\widetilde{\mathcal{A}}_{\varepsilon, \eta}^{h}\left(\Pi^{h} u_{\eta}^{\star}, \widetilde{w}_{h}\right)-\mathcal{A}_{\eta}^{\star}\left(u_{\eta}^{\star}, \widetilde{w}_{h}\right)\right|+\left|b\left(\widetilde{w}_{h}\right)-\widetilde{b}_{h}\left(\widetilde{w}_{h}\right)\right| \\
\leq & \left\|A_{\eta}\right\|_{L^{\infty}}\left\|u_{\eta}^{\varepsilon}-\mathcal{R}^{\varepsilon}\left(\Pi^{h} u_{\eta}^{\star}\right)\right\|_{H_{h}^{1}}\left\|w_{h}\right\|_{H_{h}^{1}}+\left|\widetilde{\mathcal{A}}_{\varepsilon, \eta}^{h}\left(\Pi^{h} u_{\eta}^{\star}, \widetilde{w}_{h}\right)-\mathcal{A}_{\eta}^{\star}\left(\Pi^{h} u_{\eta}^{\star}, \widetilde{w}_{h}\right)\right| \\
& \quad+\left\|A_{\eta}^{\star}\right\|\left\|u_{\eta}^{\star}-\Pi^{h} u_{\eta}^{\star}\right\|_{H^{1}(\mathcal{D})}\left\|\widetilde{w}_{h}\right\|_{H^{1}(\mathcal{D})}+\left|b\left(\widetilde{w}_{h}\right)-\widetilde{b}_{h}\left(\widetilde{w}_{h}\right)\right|
\end{aligned}
$$

where we have successively used the continuity of the bilinear forms $\mathcal{A}_{\varepsilon, \eta}^{h}$ and $\mathcal{A}_{\eta}^{\star}$. Using Lemmas 4.8 and 4.9 for the second and the fourth terms respectively, we deduce that

$$
\begin{aligned}
& \left|\mathcal{A}_{\varepsilon, \eta}^{h}\left(u_{\eta}^{\varepsilon}, w_{h}\right)-b\left(w_{h}\right)\right| \leq C\left\|u_{\eta}^{\varepsilon}-\mathcal{R}^{\varepsilon}\left(\Pi^{h} u_{\eta}^{\star}\right)\right\|_{H_{h}^{1}}\left\|w_{h}\right\|_{H_{h}^{1}} \\
& \quad+C\left(\frac{\varepsilon}{h}+\eta \lambda(\omega, h, \varepsilon)+\eta^{2} \mathcal{C}(\eta)\right)\left\|\Pi^{h} u_{\eta}^{\star}\right\|_{H^{1}(\mathcal{D})}\left\|\widetilde{w}_{h}\right\|_{H^{1}(\mathcal{D})}+C\left\|u_{\eta}^{\star}-\Pi^{h} u_{\eta}^{\star}\right\|_{H^{1}(\mathcal{D})}\left\|\widetilde{w}_{h}\right\|_{H^{1}(\mathcal{D})}+C \varepsilon\left\|\widetilde{w}_{h}\right\|_{H^{1}(\mathcal{D})}
\end{aligned}
$$

hence, using (4.54) and (4.74),

$$
\frac{\left|\mathcal{A}_{\varepsilon, \eta}^{h}\left(u_{\eta}^{\varepsilon}, w_{h}\right)-b\left(w_{h}\right)\right|}{\left\|w_{h}\right\|_{H_{h}^{1}}} \leq C\left\|u_{\eta}^{\varepsilon}-\mathcal{R}^{\varepsilon}\left(\Pi^{h} u_{\eta}^{\star}\right)\right\|_{H_{h}^{1}}+C\left(h+\frac{\varepsilon}{h}+\eta \lambda(\omega, h, \varepsilon)+\varepsilon+\eta^{2} \mathcal{C}(\eta)\right) .
$$

The first term is bounded as in Step 2:

$$
\left\|u_{\eta}^{\varepsilon}-\mathcal{R}^{\varepsilon}\left(\Pi^{h} u_{\eta}^{\star}\right)\right\|_{H_{h}^{1}} \leq\left\|u_{\eta}^{\varepsilon}-v_{\eta}^{\varepsilon}\right\|_{H^{1}(\mathcal{D})}+\left\|v_{\eta}^{\varepsilon}-\mathcal{R}^{\varepsilon}\left(u_{\eta}^{\star}\right)\right\|_{H_{h}^{1}}+\left\|\mathcal{R}^{\varepsilon}\left(u_{\eta}^{\star}\right)-\mathcal{R}^{\varepsilon}\left(\Pi^{h} u_{\eta}^{\star}\right)\right\|_{H_{h}^{1}},
$$

hence, using (4.28), (4.73) and (4.76), and assuming that $\varepsilon \ln (1 / \varepsilon) \leq 1$, we have

$$
\sqrt{\mathbb{E}\left[\left\|u_{\eta}^{\varepsilon}-\mathcal{R}^{\varepsilon}\left(\Pi^{h} u_{\eta}^{\star}\right)\right\|_{H_{h}^{1}}^{2}\right]} \leq C\left(\sqrt{\varepsilon}+\eta+\frac{\varepsilon}{h}+h\right) .
$$


Collecting (4.78) and (4.79), we thus obtain

$$
\sqrt{\mathbb{E}\left[\left(\sup _{w_{h} \in \mathcal{W}_{h}} \frac{\left|\mathcal{A}_{\varepsilon, \eta}^{h}\left(u_{\eta}^{\varepsilon}, w_{h}\right)-b\left(w_{h}\right)\right|}{\left\|w_{h}\right\|_{H_{h}^{1}}}\right)^{2}\right]} \leq C\left(h+\frac{\varepsilon}{h}+\eta \sqrt{\mathbb{E}\left[\lambda^{2}(\cdot, h, \varepsilon)\right]}+\sqrt{\varepsilon}+\eta+\eta^{2} \mathcal{C}(\eta)\right) .
$$

Step 4. Collecting (4.38), (4.77) and (4.80), we get

$$
\sqrt{\mathbb{E}\left[\left\|u_{\eta}^{\varepsilon}-u_{S}\right\|_{H_{h}^{1}}^{2}\right]} \leq C\left(\sqrt{\varepsilon}+h+\frac{\varepsilon}{h}+\eta \sqrt{\mathbb{E}\left[\lambda^{2}(\cdot, h, \varepsilon)\right]}+\eta+\eta^{2} \mathcal{C}(\eta)\right)
$$

where $C$ is a constant independent of $\varepsilon, h$ and $\eta$, and $\mathcal{C}$ is a bounded function as $\eta$ goes to 0 . Using (4.42), we deduce that

$$
\sqrt{\mathbb{E}\left[\left\|u_{\eta}^{\varepsilon}-u_{S}\right\|_{H_{h}^{1}}^{2}\right]} \leq C\left(\sqrt{\varepsilon}+h+\frac{\varepsilon}{h}+\eta\left(\frac{\varepsilon}{h}\right)^{d / 2} \ln (N(h))+\eta+\eta^{2} \mathcal{C}(\eta)\right)
$$

where $N(h)$ is the number of elements $\mathbf{K}$ in the domain (which is of order $h^{-d}$ in dimension $d$ ). This concludes the proof of Theorem 4.5.

\subsection{The one dimensional case}

In this section, we briefly consider the one dimensional situation. As in the multi-dimensional case, we assume here that $a_{\eta}^{\varepsilon}(x, \omega)=a_{\eta}\left(\frac{x}{\varepsilon}, \omega\right)$, where $a_{\eta}$ is a stationary random function satisfying, for any $|\eta| \leq 1$, the condition $0<a_{-} \leq a_{\eta}(x, \omega) \leq a^{+}$almost everywhere in $\mathbb{R}$, almost surely. In line with (4.6), we assume that

$$
a_{\eta}(x, \omega)=a_{\text {per }}(x)+\eta a_{1}(x, \omega),
$$

where $\eta$ is a small parameter $(|\eta| \leq 1), a_{\text {per }}$ is a 1-periodic function satisfying the condition $0<a_{-} \leq a_{\text {per }}(x) \leq$ $a^{+}$almost everywhere on $\mathbb{R}$, and $a_{1}$ is a bounded stationary random function: $\left|a_{1}(x, \omega)\right| \leq C$ almost everywhere in $\mathbb{R}$, almost surely. In the vein of (4.7), we suppose that

$$
a_{1}(x, \omega)=\sum_{k \in \mathbb{Z}} \mathbf{1}_{(k, k+1]}(x) X_{k}(\omega) b_{\text {per }}(x) \quad \text { such that } \quad \exists C, \forall k \in \mathbb{Z}, \quad\left|X_{k}(\omega)\right| \leq C \quad \text { almost surely, }
$$

where $\left(X_{k}(\omega)\right)_{k \in \mathbb{Z}}$ is a sequence of i.i.d. scalar random variables and $b_{\text {per }} \in L^{\infty}(\mathbb{R})$ is a 1-periodic function.

Note that, in this one-dimensional setting, we do not make any regularity assumption on $a_{\text {per }}$ (in the vein of (4.8)). In the multi-dimensional case, this assumption is useful to e.g. state that the periodic corrector satisfies $w_{p}^{0} \in W^{1, \infty}\left(\mathbb{R}^{d}\right)$ for any $p \in \mathbb{R}^{d}$. In the one-dimensional case, the corrector problem can be solved analytically, and one can see that the above assumption $a_{\text {per }}(x) \geq a_{-}>0$ almost everywhere on $\mathbb{R}$ is sufficient to obtain such regularity on the corrector. Similarly, we do not need to assume here, in contrast to Theorem 4.5, that $u_{0}^{\star}$ and $\bar{u}_{1}^{\star}$ defined by (4.20) and (4.21) both belong to $W^{2, \infty}(\mathcal{D})$ (an assumption equivalent to $f \in L^{\infty}(\mathcal{D}$ ), in the present one-dimensional setting). The assumption $f \in L^{2}(\mathcal{D})$ is sufficient.

The problem (4.4) now reads

$$
-\frac{\mathrm{d}}{\mathrm{d} x}\left(a_{\eta}\left(\frac{x}{\varepsilon}, \omega\right) \frac{\mathrm{d}}{\mathrm{d} x} u_{\eta}^{\varepsilon}(x, \omega)\right)=f(x) \quad \text { in }(0,1), \quad u_{\eta}^{\varepsilon}(0, \omega)=u_{\eta}^{\varepsilon}(1, \omega)=0 .
$$

We consider a uniform discretization of the interval $(0,1)$ in the elements $\mathbf{K}_{i}=\left(x_{i}, x_{i+1}\right)$, with $x_{i+1}-x_{i}=h=$ $1 / L$ for some $L \in \mathbb{N}^{\star}$. 
The one-dimensional version of Theorem 4.5 reads as follows:

Theorem 4.13. In the one-dimensional setting, assume that $a_{\eta}^{\varepsilon}$ satisfies (4.81)-(4.82). Let $u_{\eta}^{\varepsilon}$ be the solution to (4.83) with $f \in L^{2}(0,1)$, and $u_{S}$ be the weakly stochastic MsFEM solution to (4.32). Suppose that $h / \varepsilon \in \mathbb{N}^{\star}$. We then have

$$
\sqrt{\mathbb{E}\left[\left\|u_{\eta}^{\varepsilon}-u_{S}\right\|_{H_{h}^{1}}^{2}\right]} \leq C\left(\varepsilon+h+\eta\left(\frac{\varepsilon}{h}\right)^{1 / 2} \ln (1 / h)+\eta+\eta^{2} \mathcal{C}(\eta)\right)
$$

where $C$ is a constant independent of $\varepsilon, h$ and $\eta$ and $\mathcal{C}$ is a bounded function as $\eta$ goes to 0.

Proof. The proof of this result follows the same lines as that for the multi-dimensional case. It is based upon the homogenization result contained in Theorem 4.3 above.

As pointed out above, the rate of convergence stated in Theorem 4.2 (and hence the estimate provided by Thm. 4.5) is not optimal in dimension one. This hence motivates Theorems 4.3 and 4.13 , which are their respective one-dimensional variants. On another note, the assumption $h / \varepsilon \in \mathbb{N}^{\star}$ implies that some terms in the error bound vanish. A result similar to (4.84) holds in the absence of such assumption, with the additional term $\varepsilon / h$ in the right-hand side.

\section{Appendix A. Proofs of Lemmas 4.8, 4.9 And 4.11}

Proof of Lemma 4.8. This result relies on the expansion

$$
\phi_{j}^{\varepsilon, \mathbf{K}}(x)=\phi_{j}^{0, \mathbf{K}}(x)+\varepsilon \sum_{m=1}^{d}\left(w_{e_{m}}^{0}\left(\frac{x}{\varepsilon}\right)-\left.\xi_{\varepsilon}^{m}(x)\right|_{\mathbf{K}}\right) \partial_{m} \phi_{j}^{0, \mathbf{K}}
$$

from (4.50) and the fact that $\nabla \phi_{j}^{0, \mathbf{K}}$ is constant on $\mathbf{K}$.

For any $v_{h}$ and $w_{h}$ in $\mathcal{V}_{h}$, we write

$$
\left|\mathcal{A}_{\eta}^{\star}\left(v_{h}, w_{h}\right)-\widetilde{\mathcal{A}}_{\varepsilon, \eta}^{h}\left(v_{h}, w_{h}\right)\right|=\left|\sum_{\mathbf{K} \in \mathcal{T}_{h}}\left(\int_{\mathbf{K}}\left(\nabla w_{h}\right)^{T} A_{\eta}^{\star} \nabla v_{h}-\sum_{i, j=1}^{L} v_{h}^{j} w_{h}^{i} \int_{\mathbf{K}}\left(\nabla \phi_{i}^{\varepsilon, \mathbf{K}}\right)^{T} A_{\eta}\left(\frac{x}{\varepsilon}, \omega\right) \nabla \phi_{j}^{\varepsilon, \mathbf{K}} \mathrm{d} x\right)\right|,
$$

where $v_{h}=\sum_{j=1}^{L} v_{h}^{j} \phi_{j}^{0}$ and likewise for $w_{h}$. Using the above expansion of $\phi_{j}^{\varepsilon, \mathbf{K}}$ and the fact that $\nabla \phi_{j}^{0, \mathbf{K}}$ is constant on $\mathbf{K}$, we have

$$
\begin{aligned}
& \sum_{i, j=1}^{L} v_{h}^{j} w_{h}^{i} \int_{\mathbf{K}}\left(\nabla \phi_{i}^{\varepsilon, \mathbf{K}}\right)^{T} A_{\eta}\left(\frac{x}{\varepsilon}, \omega\right) \nabla \phi_{j}^{\varepsilon, \mathbf{K}} \mathrm{d} x \\
= & \sum_{m, p=1}^{d} \frac{1}{|\mathbf{K}|} \int_{\mathbf{K}}\left[e_{p}+\nabla w_{e_{p}}^{0}\left(\frac{x}{\varepsilon}\right)-\varepsilon \nabla \xi_{\varepsilon}^{p}(x)\right]^{T} A_{\eta}\left(\frac{x}{\varepsilon}, \omega\right)\left[e_{m}+\nabla w_{e_{m}}^{0}\left(\frac{x}{\varepsilon}\right)-\varepsilon \nabla \xi_{\varepsilon}^{m}(x)\right] \mathrm{d} x \\
& \times \sum_{i, j=1}^{L} v_{h}^{j} w_{h}^{i} \int_{\mathbf{K}} \partial_{p} \phi_{i}^{0, \mathbf{K}} \partial_{m} \phi_{j}^{0, \mathbf{K}} \\
= & \sum_{m, p=1}^{d} \frac{1}{|\mathbf{K}|} \int_{\mathbf{K}}\left[e_{p}+\nabla w_{e_{p}}^{0}\left(\frac{x}{\varepsilon}\right)-\varepsilon \nabla \xi_{\varepsilon}^{p}(x)\right]^{T} A_{\eta}\left(\frac{x}{\varepsilon}, \omega\right)\left[e_{m}+\nabla w_{e_{m}}^{0}\left(\frac{x}{\varepsilon}\right)-\varepsilon \nabla \xi_{\varepsilon}^{m}(x)\right] \mathrm{d} x \int_{\mathbf{K}} \partial_{m} v_{h} \partial_{p} w_{h} .
\end{aligned}
$$

We thus obtain

$$
\left|\mathcal{A}_{\eta}^{\star}\left(v_{h}, w_{h}\right)-\widetilde{\mathcal{A}}_{\varepsilon, \eta}^{h}\left(v_{h}, w_{h}\right)\right|=\left|\sum_{\mathbf{K}} \sum_{m, p=1}^{d} \Lambda_{m p}^{\mathbf{K}} \int_{\mathbf{K}} \partial_{m} v_{h} \partial_{p} w_{h}\right| \leq \sum_{\mathbf{K}}\left\|v_{h}\right\|_{H^{1}(\mathbf{K})}\left\|w_{h}\right\|_{H^{1}(\mathbf{K})} \sum_{m, p=1}^{d}\left|\Lambda_{m p}^{\mathbf{K}}\right|,
$$


where

$$
\Lambda_{m p}^{\mathbf{K}}=\left[A_{\eta}^{\star}\right]_{m p}-\frac{1}{|\mathbf{K}|} \int_{\mathbf{K}}\left[e_{p}+\nabla w_{e_{p}}^{0}\left(\frac{x}{\varepsilon}\right)-\varepsilon \nabla \xi_{\varepsilon}^{p}(x)\right]^{T} A_{\eta}\left(\frac{x}{\varepsilon}, \omega\right)\left[e_{m}+\nabla w_{e_{m}}^{0}\left(\frac{x}{\varepsilon}\right)-\varepsilon \nabla \xi_{\varepsilon}^{m}(x)\right] \mathrm{d} x,
$$

which we write

$$
\Lambda_{m p}^{\mathbf{K}}=D_{0}+D_{1}-D_{2},
$$

with

$$
\begin{aligned}
D_{0} & =\left[A_{\eta}^{\star}\right]_{m p}-\frac{1}{|\mathbf{K}|} \int_{\mathbf{K}}\left[e_{p}+\nabla w_{e_{p}}^{0}\left(\frac{x}{\varepsilon}\right)\right]^{T} A_{\eta}\left(\frac{x}{\varepsilon}, \omega\right)\left[e_{m}+\nabla w_{e_{m}}^{0}\left(\frac{x}{\varepsilon}\right)\right] \mathrm{d} x, \\
D_{1} & =\frac{\varepsilon}{|\mathbf{K}|}\left(\int_{\mathbf{K}}\left[e_{p}+\nabla w_{e_{p}}^{0}\left(\frac{\dot{\varepsilon}}{\varepsilon}\right)\right]^{T} A_{\eta}\left(\frac{\dot{-}}{\varepsilon}, \omega\right) \nabla \xi_{\varepsilon}^{m}+\int_{\mathbf{K}}\left(\nabla \xi_{\varepsilon}^{p}(x)\right)^{T} A_{\eta}\left(\frac{x}{\varepsilon}, \omega\right)\left[e_{m}+\nabla w_{e_{m}}^{0}\left(\frac{x}{\varepsilon}\right)\right] \mathrm{d} x\right), \\
D_{2} & =\frac{\varepsilon^{2}}{|\mathbf{K}|} \int_{\mathbf{K}}\left(\nabla \xi_{\varepsilon}^{p}(x)\right)^{T} A_{\eta}\left(\frac{x}{\varepsilon}, \omega\right) \nabla \xi_{\varepsilon}^{m}(x) \mathrm{d} x .
\end{aligned}
$$

We are thus left with bounding $\left|\Lambda_{m p}^{\mathrm{K}}\right|$ from above. We first bound $D_{1}$ and $D_{2}$. Using Lemma 4.12 (recall that $A_{\text {per satisfies }}(4.8)$, i.e. is Hölder continuous) and the fact that $w_{e_{p}}^{0} \in H^{1}(Q)$ and is $Q$-periodic, we obtain

$$
\left|D_{2}\right| \leq C \frac{\varepsilon^{2}}{h^{2}}
$$

and

$$
\begin{aligned}
\left|\frac{\varepsilon}{|\mathbf{K}|} \int_{\mathbf{K}}\left[e_{p}+\nabla w_{e_{p}}^{0}\left(\frac{x}{\varepsilon}\right)\right]^{T} A_{\eta}\left(\frac{x}{\varepsilon}, \omega\right) \nabla \xi_{\varepsilon}^{m}(x) \mathrm{d} x\right| & \leq \frac{\varepsilon}{|\mathbf{K}|}\left\|A_{\eta}\right\|_{L^{\infty}} \frac{C}{h} \int_{\mathbf{K}}\left|e_{p}+\nabla w_{e_{p}}^{0}\left(\frac{x}{\varepsilon}\right)\right| \mathrm{d} x \\
& \leq C \frac{\varepsilon}{|\mathbf{K}| h}\left(|\mathbf{K}|+\varepsilon^{d} \int_{\mathbf{K} / \varepsilon}\left|\nabla w_{e_{p}}^{0}(y)\right| \mathrm{d} y\right) \\
& \leq C \frac{\varepsilon}{h}
\end{aligned}
$$

hence

$$
\left|D_{1}\right| \leq C \frac{\varepsilon}{h},
$$

where $C$ is a deterministic constant independent of $h, \varepsilon$ and $\eta$. We next turn to $D_{0}$. We introduce the cells $Q_{i}^{\varepsilon}=\varepsilon(Q+i), i \in \mathbb{Z}^{d}$, let $I_{\mathbf{K}}^{\varepsilon}=\bigcup_{Q_{i}^{\varepsilon} \subset \mathbf{K}} Q_{i}^{\varepsilon}$, and recast (A.3) as

$$
D_{0}=D_{0}^{\text {bulk }}-D_{0}^{\text {boundary }}
$$

with

$$
\begin{aligned}
D_{0}^{\text {bulk }} & =\left[A_{\eta}^{\star}\right]_{m p}-\frac{1}{|\mathbf{K}|} \int_{I_{\mathbf{K}}^{\varepsilon}}\left[e_{p}+\nabla w_{e_{p}}^{0}\left(\frac{x}{\varepsilon}\right)\right]^{T} A_{\eta}\left(\frac{x}{\varepsilon}, \omega\right)\left[e_{m}+\nabla w_{e_{m}}^{0}\left(\frac{x}{\varepsilon}\right)\right] \mathrm{d} x, \\
D_{0}^{\text {boundary }} & =\frac{1}{|\mathbf{K}|} \int_{\mathbf{K} \backslash I_{\mathbf{K}}^{\varepsilon}}\left[e_{p}+\nabla w_{e_{p}}^{0}\left(\frac{x}{\varepsilon}\right)\right]^{T} A_{\eta}\left(\frac{x}{\varepsilon}, \omega\right)\left[e_{m}+\nabla w_{e_{m}}^{0}\left(\frac{x}{\varepsilon}\right)\right] \mathrm{d} x .
\end{aligned}
$$

We denote by $J_{\mathbf{K}}^{\varepsilon}$ the set of cells $Q_{i}^{\varepsilon}$ that intersect the element $\mathbf{K}$, i.e.

$$
J_{\mathbf{K}}^{\varepsilon}=\bigcup_{Q_{i}^{\varepsilon} \cap \mathbf{K} \neq \emptyset} Q_{i}^{\varepsilon}
$$


By construction, $I_{\mathbf{K}}^{\varepsilon} \subset \mathbf{K} \subset J_{\mathbf{K}}^{\varepsilon}$. Using that $w_{e_{p}}^{0} \in H^{1}(Q)$ and is $Q$-periodic, we write

$$
\begin{aligned}
\left|D_{0}^{\text {boundary }}\right| & \leq\left\|A_{\eta}\right\|_{L^{\infty}} \frac{1}{|\mathbf{K}|} \sqrt{\int_{J_{\mathbf{K}}^{\varepsilon} \backslash I_{\mathbf{K}}^{\varepsilon}}\left[e_{p}+\nabla w_{e_{p}}^{0}\left(\frac{x}{\varepsilon}\right)\right]^{2}} \mathrm{~d} x \sqrt{\int_{J_{\mathbf{K}}^{\varepsilon} \backslash I_{\mathbf{K}}^{\varepsilon}}\left[e_{m}+\nabla w_{e_{m}}^{0}\left(\frac{x}{\varepsilon}\right)\right]^{2} \mathrm{~d} x} \\
& \leq C \frac{\varepsilon^{d}}{|\mathbf{K}|} \frac{|\partial \mathbf{K}|}{\varepsilon^{d-1}} \sqrt{\int_{Q}\left[e_{p}+\nabla w_{e_{p}}^{0}(y)\right]^{2}} \mathrm{~d} y \sqrt{\int_{Q}\left[e_{m}+\nabla w_{e_{m}}^{0}(y)\right]^{2} \mathrm{~d} y} \\
& \leq C \frac{\varepsilon}{h} .
\end{aligned}
$$

We next consider (A.7):

$$
D_{0}^{\text {bulk }}=\frac{\left|\mathbf{K} \backslash I_{\mathbf{K}}^{\varepsilon}\right|}{|\mathbf{K}|}\left[A_{\eta}^{\star}\right]_{m p}+\frac{\left|I_{\mathbf{K}}^{\varepsilon}\right|}{|\mathbf{K}|} \bar{D}_{0}^{\text {bulk }}
$$

with

$$
\bar{D}_{0}^{\mathrm{bulk}}=\left[A_{\eta}^{\star}\right]_{m p}-\frac{1}{\left|I_{\mathbf{K}}^{\varepsilon}\right|} \int_{I_{\mathbf{K}}^{\varepsilon}}\left[e_{p}+\nabla w_{e_{p}}^{0}\left(\frac{x}{\varepsilon}\right)\right]^{T} A_{\eta}\left(\frac{x}{\varepsilon}, \omega\right)\left[e_{m}+\nabla w_{e_{m}}^{0}\left(\frac{x}{\varepsilon}\right)\right] \mathrm{d} x .
$$

Using the expansion (4.14) of $A_{\eta}^{\star}$, we write

$$
\begin{aligned}
\bar{D}_{0}^{\text {bulk }}= & \int_{Q}\left[e_{p}+\nabla w_{e_{p}}^{0}(y)\right]^{T} A_{\text {per }}(y)\left[e_{m}+\nabla w_{e_{m}}^{0}(y)\right] \mathrm{d} y \\
& -\frac{1}{\left|I_{\mathbf{K}}^{\varepsilon}\right|} \int_{I_{\mathbf{K}}^{\varepsilon}}\left[e_{p}+\nabla w_{e_{p}}^{0}\left(\frac{x}{\varepsilon}\right)\right]^{T} A_{\text {per }}\left(\frac{x}{\varepsilon}\right)\left[e_{m}+\nabla w_{e_{m}}^{0}\left(\frac{x}{\varepsilon}\right)\right] \mathrm{d} x \\
& +\eta\left(\int_{Q}\left[e_{p}+\nabla w_{e_{p}}^{0}(y)\right]^{T} \mathbb{E}\left(A_{1}(y, \cdot)\right)\left[e_{m}+\nabla w_{e_{m}}^{0}(y)\right] \mathrm{d} y\right. \\
& \left.-\frac{1}{\left|I_{\mathbf{K}}^{\varepsilon}\right|} \int_{I_{\mathbf{K}}^{\varepsilon}}\left[e_{p}+\nabla w_{e_{p}}^{0}\left(\frac{x}{\varepsilon}\right)\right]^{T} A_{1}\left(\frac{x}{\varepsilon}, \omega\right)\left[e_{m}+\nabla w_{e_{m}}^{0}\left(\frac{x}{\varepsilon}\right)\right] \mathrm{d} x\right)+\eta^{2} \mathcal{C}(\eta) .
\end{aligned}
$$

The leading order term in (A.10) vanishes. We are hence left with

$$
\bar{D}_{0}^{\text {bulk }}=\frac{\eta}{\left|I_{\mathbf{K}}^{\varepsilon}\right|} \int_{I_{\mathbf{K}}^{\varepsilon}}\left[e_{p}+\nabla w_{e_{p}}^{0}\left(\frac{x}{\varepsilon}\right)\right]^{T}\left(\mathbb{E}\left(A_{1}\left(\frac{x}{\varepsilon}, \cdot\right)\right)-A_{1}\left(\frac{x}{\varepsilon}, \omega\right)\right)\left[e_{m}+\nabla w_{e_{m}}^{0}\left(\frac{x}{\varepsilon}\right)\right] \mathrm{d} x+\eta^{2} \mathcal{C}(\eta)
$$

Collecting (A.2), (A.4), (A.5), (A.6), (A.8), (A.9) and (A.11), together with the fact that $\frac{\left|\mathbf{K} \backslash I_{\mathbf{K}}^{\varepsilon}\right|}{|\mathbf{K}|}\left|\left[A_{\eta}^{\star}\right]_{m p}\right| \leq$ $C \frac{\varepsilon}{h}$, we obtain

$$
\left|\Lambda_{m p}^{\mathbf{K}}\right| \leq C \frac{\varepsilon}{h}+\frac{\eta}{\left|I_{\mathbf{K}}^{\varepsilon}\right|}\left|\int_{I_{\mathbf{K}}^{\varepsilon}}\left[e_{p}+\nabla w_{e_{p}}^{0}\left(\frac{x}{\varepsilon}\right)\right]^{T}\left(\mathbb{E}\left(A_{1}\left(\frac{x}{\varepsilon}, \cdot\right)\right)-A_{1}\left(\frac{x}{\varepsilon}, \omega\right)\right)\left[e_{m}+\nabla w_{e_{m}}^{0}\left(\frac{x}{\varepsilon}\right)\right] \mathrm{d} x\right|+\eta^{2} \mathcal{C}(\eta) .
$$

We set

$$
\lambda(\omega, h, \varepsilon)=\max _{\mathbf{K} \in \mathcal{T}_{h}} \max _{1 \leq m, p \leq d}\left|\frac{1}{\left|I_{\mathbf{K}}^{\varepsilon}\right|} \int_{I_{\mathbf{K}}^{\varepsilon}}\left[e_{p}+\nabla w_{e_{p}}^{0}\left(\frac{x}{\varepsilon}\right)\right]^{T}\left(\mathbb{E}\left(A_{1}\left(\frac{x}{\varepsilon}, \cdot\right)\right)-A_{1}\left(\frac{x}{\varepsilon}, \omega\right)\right)\left[e_{m}+\nabla w_{e_{m}}^{0}\left(\frac{x}{\varepsilon}\right)\right] \mathrm{d} x\right| .
$$

We thus have, for any $\mathbf{K}, \sum_{m, p=1}^{d}\left|\Lambda_{m p}^{\mathbf{K}}\right| \leq C\left(\frac{\varepsilon}{h}+\eta \lambda(\omega, h, \varepsilon)+\eta^{2} \mathcal{C}(\eta)\right)$. Using (A.1), we thus obtain that

$$
\left|\mathcal{A}_{\eta}^{\star}\left(v_{h}, w_{h}\right)-\widetilde{\mathcal{A}}_{\varepsilon, \eta}^{h}\left(v_{h}, w_{h}\right)\right| \leq C\left(\frac{\varepsilon}{h}+\eta \lambda(\omega, h, \varepsilon)+\eta^{2} \mathcal{C}(\eta)\right)\left\|v_{h}\right\|_{H^{1}(\mathcal{D})}\left\|w_{h}\right\|_{H^{1}(\mathcal{D})} .
$$

This concludes the proof of Lemma 4.8. 
Proof of Lemma 4.9. Again, as for Lemma 4.8, this result relies on the expansion (4.50) of $\phi_{i}^{\varepsilon, \mathbf{K}}$ and the fact that $\nabla \phi_{i}^{0, \mathbf{K}}$ is constant on $\mathbf{K}$.

Setting $w_{h}(x)=\sum_{i} w_{h}^{i} \phi_{i}^{0}(x)$, we observe that

$$
\left|\widetilde{b}_{h}\left(w_{h}\right)-b\left(w_{h}\right)\right|=\left|\sum_{\mathbf{K}} \sum_{i=1}^{L} w_{h}^{i} \int_{\mathbf{K}} f(x)\left(\phi_{i}^{\varepsilon, \mathbf{K}}(x)-\phi_{i}^{0, \mathbf{K}}(x)\right) \mathrm{d} x\right| .
$$

Using (4.50) and the fact that $\nabla \phi_{i}^{0, \mathbf{K}}$ is constant on $\mathbf{K}$, we obtain

$$
\sum_{i=1}^{L} w_{h}^{i} \int_{\mathbf{K}} f(x)\left(\phi_{i}^{\varepsilon, \mathbf{K}}(x)-\phi_{i}^{0, \mathbf{K}}(x)\right) \mathrm{d} x=\sum_{p=1}^{d} \frac{\varepsilon}{|\mathbf{K}|} \int_{\mathbf{K}} f(x)\left(w_{e_{p}}^{0}\left(\frac{x}{\varepsilon}\right)-\xi_{\varepsilon}^{p}(x)\right) \mathrm{d} x \sum_{i=1}^{L} w_{h}^{i} \int_{\mathbf{K}} \partial_{p} \phi_{i}^{0, \mathbf{K}} .
$$

We have

$$
\left|\sum_{i=1}^{L} w_{h}^{i} \int_{\mathbf{K}} \partial_{p} \phi_{i}^{0, \mathbf{K}}\right|=\left|\int_{\mathbf{K}} \partial_{p} w_{h}\right| \leq \sqrt{|\mathbf{K}|}\left\|w_{h}\right\|_{H^{1}(\mathbf{K})}
$$

and

$$
\begin{aligned}
\left|\int_{\mathbf{K}} f(x)\left(w_{e_{p}}^{0}\left(\frac{x}{\varepsilon}\right)-\xi_{\varepsilon}^{p}(x)\right) \mathrm{d} x\right| & \leq\|f\|_{L^{2}(\mathbf{K})}\left(\left\|w_{e_{p}}^{0}\left(\frac{\dot{\varepsilon}}{\varepsilon}\right)\right\|_{L^{2}(\mathbf{K})}+\left\|\xi_{\varepsilon}^{p}\right\|_{L^{2}(\mathbf{K})}\right) \\
& \leq\|f\|_{L^{2}(\mathbf{K})} \sqrt{|\mathbf{K}|}\left(\left\|w_{e_{p}}^{0}\right\|_{L^{\infty}\left(\mathbb{R}^{d}\right)}+\left\|\xi_{\varepsilon}^{p}\right\|_{L^{\infty}(\mathbf{K})}\right) .
\end{aligned}
$$

Recall now that, since $A_{\text {per }}$ satisfies (4.8) (i.e. is Hölder continuous), we know that $\xi_{\varepsilon}^{p}$ and $w_{e_{p}}^{0}$ are both continuous, and that $w_{e_{p}}^{0} \in L^{\infty}\left(\mathbb{R}^{d}\right)$. Using the maximum principle on (4.49), we write

$$
\left\|\xi_{\varepsilon}^{p}\right\|_{L^{\infty}(\mathbf{K})} \leq\left\|w_{e_{p}}^{0}\right\|_{L^{\infty}(\partial \mathbf{S})} \leq\left\|w_{e_{p}}^{0}\right\|_{L^{\infty}\left(\mathbb{R}^{d}\right)},
$$

and we thus deduce from (A.14) that

$$
\left|\int_{\mathbf{K}} f(x)\left(w_{e_{p}}^{0}\left(\frac{x}{\varepsilon}\right)-\xi_{\varepsilon}^{p}(x)\right) \mathrm{d} x\right| \leq C\|f\|_{L^{2}(\mathbf{K})} \sqrt{|\mathbf{K}|}
$$

for a constant $C$ independent of $h$ and $\varepsilon$. Collecting (A.12), (A.13) and (A.15), we obtain

$$
\left|\widetilde{b}_{h}\left(w_{h}\right)-b\left(w_{h}\right)\right| \leq C \varepsilon\|f\|_{L^{2}(\mathcal{D})}\left\|w_{h}\right\|_{H^{1}(\mathcal{D})} .
$$

This concludes the proof of Lemma 4.9 .

Proof of Lemma 4.11. We first prove the uniform bound on $\lambda$. Recall that the field $A_{1}$ is bounded almost surely and almost everywhere. This implies that

$$
\begin{aligned}
\mid \frac{1}{\left|I_{\mathbf{K}}^{\varepsilon}\right|} \int_{I_{\mathbf{K}}^{\varepsilon}}\left[e_{p}+\nabla w_{e_{p}}^{0}\left(\frac{x}{\varepsilon}\right)\right]^{T}\left(A_{1}\left(\frac{x}{\varepsilon}, \omega\right)\right. & \left.-\mathbb{E}\left(A_{1}\left(\frac{x}{\varepsilon}, \cdot\right)\right)\right)\left[e_{m}+\nabla w_{e_{m}}^{0}\left(\frac{x}{\varepsilon}\right)\right] \mathrm{d} x \mid \\
& \leq 2\left\|A_{1}\right\|_{L^{\infty}} \frac{1}{\left|I_{\mathbf{K}}^{\varepsilon}\right|}\left\|e_{p}+\nabla w_{e_{p}}^{0}\left(\frac{\cdot}{\varepsilon}\right)\right\|_{L^{2}\left(I_{\mathbf{K}}^{\varepsilon}\right)}\left\|e_{m}+\nabla w_{e_{m}}^{0}\left(\frac{\cdot}{\varepsilon}\right)\right\|_{L^{2}\left(I_{\mathbf{K}}^{\varepsilon}\right)} .
\end{aligned}
$$

Then, using the $Q$-periodicity of $w_{e_{p}}^{0}$, we obtain

$$
\left\|e_{p}+\nabla w_{e_{p}}^{0}\left(\frac{\cdot}{\varepsilon}\right)\right\|_{L^{2}\left(I_{\mathbf{K}}^{\varepsilon}\right)}^{2}=\sum_{Q_{i}^{\varepsilon} \subset I_{\mathbf{K}}^{\varepsilon}} \int_{Q_{i}^{\varepsilon}}\left[e_{p}+\nabla w_{e_{p}}^{0}\left(\frac{x}{\varepsilon}\right)\right]^{2} \mathrm{~d} x=\left|I_{\mathbf{K}}^{\varepsilon}\right|\left\|e_{p}+\nabla w_{e_{p}}^{0}\right\|_{L^{2}(Q)}^{2} .
$$


We thus have

$$
\lambda(\omega, h, \varepsilon) \leq 2\left\|A_{1}\right\|_{L^{\infty}} \max _{1 \leq p, m \leq d}\left[\left\|e_{p}+\nabla w_{e_{p}}^{0}\right\|_{L^{2}(Q)}\left\|e_{m}+\nabla w_{e_{m}}^{0}\right\|_{L^{2}(Q)}\right],
$$

hence $\lambda(\omega, h, \varepsilon)$ is bounded almost surely by a deterministic constant independent of $h$ and $\varepsilon$.

We next turn to (4.42). Rewrite (4.40) as

$$
\lambda(\omega, h, \varepsilon)=\max _{\mathbf{K}} \max _{1 \leq m, p \leq d}\left|S_{\mathbf{K}}^{m, p}\right|,
$$

with

$$
S_{\mathbf{K}}^{m, p}:=\frac{1}{\left|I_{\mathbf{K}}^{\varepsilon}\right|} \int_{I_{\mathbf{K}}^{\varepsilon}}\left[e_{p}+\nabla w_{e_{p}}^{0}\left(\frac{x}{\varepsilon}\right)\right]^{T}\left(A_{1}\left(\frac{x}{\varepsilon}, \omega\right)-\mathbb{E}\left(A_{1}\left(\frac{x}{\varepsilon}, \cdot\right)\right)\left[e_{m}+\nabla w_{e_{m}}^{0}\left(\frac{x}{\varepsilon}\right)\right] \mathrm{d} x .\right.
$$

Using the periodicity of the correctors $w_{p}^{0}$ and the specific form (4.7) of $A_{1}$, we have

$$
S_{\mathbf{K}}^{m, p}=\tau^{m, p} \frac{1}{N_{\mathbf{K}}} \sum_{i ; Q_{i}^{\varepsilon} \subset I_{\mathbf{K}}^{\varepsilon}} \frac{X_{i}-\mathbb{E}\left(X_{0}\right)}{\sqrt{\operatorname{Var}\left(X_{0}\right)}}
$$

with

$$
\tau^{m, p}=\sqrt{\operatorname{Var}\left(X_{0}\right)} \int_{Q}\left[e_{p}+\nabla w_{e_{p}}^{0}(y)\right]^{T} B_{\mathrm{per}}(y)\left[e_{m}+\nabla w_{e_{m}}^{0}(y)\right] \mathrm{d} y \quad \text { and } \quad N_{\mathbf{K}}=\operatorname{Card}\left\{i ; Q_{i}^{\varepsilon} \subset I_{\mathbf{K}}^{\varepsilon}\right\} .
$$

Thus, $\lambda(\omega, h, \varepsilon)$ reads

$$
\lambda(\omega, h, \varepsilon)=\gamma \max _{\mathbf{K}}\left|S_{\mathbf{K}}^{\varepsilon}(\omega)\right|,
$$

where $\gamma=\max _{1 \leq m, p \leq d} \tau^{m, p}$ and

$$
S_{\mathbf{K}}^{\varepsilon}(\omega)=\frac{1}{N_{\mathbf{K}}} \sum_{i ; Q_{i}^{\varepsilon} \subset I_{\mathbf{K}}^{\varepsilon}} \frac{X_{i}-\mathbb{E}\left(X_{0}\right)}{\sqrt{\operatorname{Var}\left(X_{0}\right)}}
$$

Introduce $F_{N_{\mathbf{K}}}(x)=\mathbb{P}\left(\sqrt{N_{\mathbf{K}}}\left|S_{\mathbf{K}}^{\varepsilon}\right| \leq x\right)$, and let $\varphi_{N_{\mathbf{K}}}$ be the probability density function of the random variable $\sqrt{N_{\mathbf{K}}}\left|S_{\mathbf{K}}^{\varepsilon}(\omega)\right|$. Using the assumption that each element $\mathbf{K}$ contains a number $N_{\mathbf{K}}$ of cells of size $\varepsilon$ that satisfies $N_{\mathbf{K}} \geq \alpha\left(\frac{h}{\varepsilon}\right)^{d}$ for some $\alpha>0$, independent of $\mathbf{K}, h$ and $\varepsilon$, we write

$$
\mathbb{E}\left(\alpha \frac{h^{d}}{\varepsilon^{d}} \frac{\lambda^{2}(\cdot, h, \varepsilon)}{\gamma^{2}}\right) \leq \mathbb{E}\left(N_{\mathbf{K}} \max _{\mathbf{K}}\left|S_{\mathbf{K}}^{\varepsilon}\right|^{2}\right)=\int_{0}^{\infty} x^{2} \frac{\mathrm{d}}{\mathrm{d} x} \mathbb{P}\left(\sqrt{N_{\mathbf{K}}} \max _{\mathbf{K}}\left|S_{\mathbf{K}}^{\varepsilon}\right| \leq x\right) \mathrm{d} x .
$$

Since

$$
\mathbb{P}\left(\sqrt{N_{\mathbf{K}}} \max _{\mathbf{K}}\left|S_{\mathbf{K}}^{\varepsilon}\right| \leq x\right)=\left[\mathbb{P}\left(\sqrt{N_{\mathbf{K}}}\left|S_{\mathbf{K}}^{\varepsilon}\right| \leq x\right)\right]^{N(h)}=\left[F_{N_{\mathbf{K}}}(x)\right]^{N(h)},
$$

we deduce that

$$
\mathbb{E}\left(\alpha \frac{h^{d}}{\varepsilon^{d}} \frac{\lambda^{2}(\cdot, h, \varepsilon)}{\gamma^{2}}\right) \leq \int_{0}^{\infty} x^{2} N(h) F_{N_{\mathbf{K}}}^{N(h)-1}(x) \varphi_{N_{\mathbf{K}}}(x) \mathrm{d} x=e_{1}+e_{2}
$$

where

$$
e_{1}=\int_{0}^{c_{h}} x^{2} N(h) F_{N_{\mathbf{K}}}^{N(h)-1}(x) \varphi_{N_{\mathbf{K}}}(x) \mathrm{d} x \quad \text { and } \quad e_{2}=\int_{c_{h}}^{\infty} x^{2} N(h) F_{N_{\mathbf{K}}}^{N(h)-1}(x) \varphi_{N_{\mathbf{K}}}(x) \mathrm{d} x,
$$

with $c_{h}=2 \ln (N(h))$. We now successively bound from above $e_{1}$ and $e_{2}$. First, integrating by part, and using that $0 \leq F_{N_{\mathrm{K}}} \leq 1$, we obtain

$$
0 \leq e_{1}=\left[x^{2} F_{N_{\mathrm{K}}}^{N(h)}(x)\right]_{x=0}^{x=c_{h}}-\int_{0}^{c_{h}} 2 x F_{N_{\mathrm{K}}}^{N(h)}(x) \mathrm{d} x \leq c_{h}^{2} .
$$


Second, again using $0 \leq F_{N_{\mathrm{K}}} \leq 1$, we get

$$
0 \leq e_{2} \leq \int_{c_{h}}^{\infty} x^{2} N(h) \varphi_{N_{\mathbf{K}}}(x) \mathrm{d} x=N(h) \mathbb{E}\left(\mathbf{1}_{\left\{\sqrt{N_{\mathbf{K}}}\left|S_{\mathbf{K}}^{\varepsilon}\right|>c_{h}\right\}} N_{\mathbf{K}}\left|S_{\mathbf{K}}^{\varepsilon}\right|^{2}\right) .
$$

Using the Cauchy-Schwartz inequality, we obtain

$$
e_{2}^{2} \leq N(h)^{2} \mathbb{E}\left[N_{\mathbf{K}}^{2}\left|S_{\mathbf{K}}^{\varepsilon}\right|^{4}\right] \mathbb{P}\left(\sqrt{N_{\mathbf{K}}}\left|S_{\mathbf{K}}^{\varepsilon}\right|>c_{h}\right) .
$$

Introduce $Y_{i}=\frac{X_{i}-\mathbb{E}\left(X_{0}\right)}{\sqrt{\operatorname{Var}\left(X_{0}\right)}}$, so that $S_{\mathbf{K}}^{\varepsilon}(\omega)=\frac{1}{N_{\mathbf{K}}} \sum_{i} Y_{i}(\omega)$. Recall now that $\left(Y_{i}\right)_{i \in \mathbb{Z}^{d}}$ is a sequence of independent identically distributed variables, with mean zero. Any such variables satisfy the bounds

$$
\forall p \in \mathbb{N}^{\star}, \quad \exists C_{p}>0, \quad \forall N \in \mathbb{N}^{\star}, \quad\left|\mathbb{E}\left[\left(\frac{1}{N} \sum_{i=1}^{N} Y_{i}\right)^{2 p}\right]\right| \leq \frac{C_{p}}{N^{p}},
$$

for a constant $C_{p}$ that depends on $p$ and the moments of $Y_{i}$, up to order $2 p$. Recall that all moments of $Y_{i}$ are well defined, as $Y_{i}$ is bounded almost surely. Thus

$$
e_{2}^{2} \leq C_{4} N(h)^{2} \mathbb{P}\left(\sqrt{N_{\mathbf{K}}}\left|S_{\mathbf{K}}^{\varepsilon}\right|>c_{h}\right) \leq C_{4} N(h)^{2}\left[\mathbb{P}\left(\sqrt{N_{\mathbf{K}}} S_{\mathbf{K}}^{\varepsilon}>c_{h}\right)+\mathbb{P}\left(-\sqrt{N_{\mathbf{K}}} S_{\mathbf{K}}^{\varepsilon}>c_{h}\right)\right] .
$$

We now recall the Markov inequality: for any positive non-decreasing function $\psi$ on $\mathbb{R}$, and any real-valued random variable $Z$, we have

$$
\forall b \in \mathbb{R}, \quad \mathbb{P}(Z \geq b) \leq \frac{\mathbb{E}(\psi(Z))}{\psi(b)} .
$$

We apply this inequality to the random variable $Z(\omega)=\sqrt{N_{\mathbf{K}}} S_{\mathbf{K}}^{\varepsilon}(\omega)$, with $\psi=\exp (t \cdot)$ for some $t \geq 0$, and $b=c_{h}$. This yields

$$
\mathbb{P}\left(\sqrt{N_{\mathbf{K}}} S_{\mathbf{K}}^{\varepsilon} \geq c_{h}\right) \leq \mathrm{e}^{-t c_{h}} \mathbb{E}\left[\exp \left(t \sqrt{N_{\mathbf{K}}} S_{\mathbf{K}}^{\varepsilon}\right)\right] \leq \mathrm{e}^{-t c_{h}}\left[\mathbb{E}\left(\exp \left(\frac{t}{\sqrt{N_{\mathbf{K}}}} Y_{0}\right)\right)\right]^{N_{\mathbf{K}}},
$$

where we have used the fact that $S_{\mathbf{K}}^{\varepsilon}$ is a sum of i.i.d. variables. Using a Taylor expansion with respect to $t$, we see that

$$
\mathbb{E}\left[\exp \left(\frac{t}{\sqrt{N_{\mathbf{K}}}} Y_{0}\right)\right]=1+\frac{t^{2}}{2 N_{\mathbf{K}}} \mathbb{E}\left(Y_{0}^{2}\right)+\frac{1}{6 N_{\mathbf{K}}^{3 / 2}} \mathbb{E}\left[Y_{0}^{3} \exp \left(\xi Y_{0} / \sqrt{N_{\mathbf{K}}}\right)\right] \quad \text { for some } \xi \in(0, t) .
$$

Thus

$$
\left[\mathbb{E}\left(\exp \left(\frac{t}{\sqrt{N_{\mathbf{K}}}} Y_{0}\right)\right)\right]^{N_{\mathbf{K}}} \leq \exp \left[\frac{t^{2}}{2} \mathbb{E}\left(Y_{0}^{2}\right)+\frac{1}{6 \sqrt{N_{\mathbf{K}}}} \mathbb{E}\left(Y_{0}^{3} \exp \left(\xi Y_{0} / \sqrt{N_{\mathbf{K}}}\right)\right)\right]
$$

Using (A.20), taking $t=1$, and using that $\mathrm{e}^{-c_{h}}=\frac{1}{N(h)^{2}}$, we obtain

$$
\begin{aligned}
\mathbb{P}\left(\sqrt{N_{\mathbf{K}}} S_{\mathbf{K}}^{\varepsilon} \geq c_{h}\right) & \leq \frac{1}{N(h)^{2}} \exp \left[\frac{1}{2} \mathbb{E}\left(Y_{0}^{2}\right)+\frac{1}{6 \sqrt{N_{\mathbf{K}}}} \mathbb{E}\left(Y_{0}^{3} \exp \left(\xi Y_{0} / \sqrt{N_{\mathbf{K}}}\right)\right)\right] \text { for some } \xi \in(0,1), \\
& \leq \frac{1}{N(h)^{2}} \exp \left[\frac{1}{2} \mathbb{E}\left(Y_{0}^{2}\right)+\frac{1}{6} \mathbb{E}\left(\left|Y_{0}\right|^{3} \exp \left(\left|Y_{0}\right|\right)\right)\right] .
\end{aligned}
$$

Likewise, considering $Z(\omega)=-\sqrt{N_{\mathbf{K}}} S_{\mathbf{K}}^{\varepsilon}(\omega)$, we obtain a similar bound. Collecting (A.19), (A.21) and the fact that $Y_{0}$ is bounded almost surely, we have

$$
e_{2}^{2} \leq C
$$


with $C$ independent of $h$ and $\varepsilon$. Collecting (A.17), (A.18) and (A.22), we get, for a constant $C$ independent of $h$ and $\varepsilon$,

$$
\mathbb{E}\left(\lambda(\cdot, h, \varepsilon)^{2}\right) \leq C \frac{\varepsilon^{d}}{h^{d}}[\ln (N(h))]^{2} .
$$

This concludes the proof of Lemma 4.11 .

Remark A.1. The above proof shows that, when $\varepsilon \rightarrow 0$, the random variable $(h / \varepsilon)^{d / 2} \lambda(\omega, h, \varepsilon)$ converges in law to $\mathcal{G}_{h}(\omega)=\max _{\mathbf{K}}\left|G_{\mathbf{K}}(\omega)\right|$, where $G_{\mathbf{K}}(\omega)$ are i.i.d. Gaussian random variables. Precise results on the behavior of $\mathcal{G}_{h}(\omega)$ when $h \rightarrow 0$ (i.e., when the number of Gaussian random variables involved diverges) are given in e.g. [46].

Acknowledgements. Support from EOARD under Grants FA8655-10-C-4002 and FA8655-13-1-3061 is gratefully acknowledged.

\section{REFERENCES}

[1] J. Aarnes and Y.R. Efendiev, Mixed multiscale finite element methods for stochastic porous media flows. SIAM J. Sci. Comput. 30 (2009) 2319-2339.

[2] G. Allaire and M. Amar, Boundary layer tails in periodic homogenization. ESAIM: COCV 4 (1999) $209-243$.

[3] G. Allaire and R. Brizzi, A multiscale finite element method for numerical homogenization. SIAM Multiscale Model. Simul. 4 (2005) 790-812.

[4] A. Anantharaman, Ph.D. thesis, Thèse de l'Université Paris-Est (2011). Available at http://tel.archives-ouvertes.fr/ tel-00558618/fr

[5] A. Anantharaman, R. Costaouec, C. Le Bris, F. Legoll and F. Thomines, Introduction to numerical stochastic homogenization and the related computational challenges: some recent developments, in Multiscale Modeling and Analysis for Materials Simulation, vol. 22 of Lect. Notes Ser., edited by W. Bao and Q. Du. Institute for Mathematical Sciences, National University of Singapore (2011) 197-272.

[6] A. Anantharaman and C. Le Bris, Homogénéisation d'un matériau périodique faiblement perturbé aléatoirement [Homogenization of a weakly randomly perturbed periodic material]. C.R. Acad. Sci. Sér. I 348 (2010) 529-534.

[7] A. Anantharaman and C. Le Bris, A numerical approach related to defect-type theories for some weakly random problems in homogenization. SIAM Multiscale Model. Simul. 9 (2011) 513-544.

[8] A. Anantharaman and C. Le Bris, Elements of mathematical foundations for a numerical approach for weakly random homogenization problems. Commun. Comput. Phys. 11 (2012) 1103-1143.

[9] M. Avellaneda and F. H. Lin, Compactness methods in the theory of homogenization. Commun. Pure Appl. Math. 40 (1987) 803-847.

[10] G. Bal, J. Garnier, S. Motsch and V. Perrier, Random integrals and correctors in homogenization. Asymptot. Anal. 59 (2008) $1-26$.

[11] G. Bal and W. Jing, Corrector theory for MsFEM and HMM in random media. SIAM Multiscale Model. Simul. 9 (2011) $1549-1587$.

[12] A. Bensoussan, J.-L. Lions and G. Papanicolaou, Asymptotic analysis for periodic structures, vol. 5 of Studies in Mathematics and its Applications. North-Holland Publishing Co., Amsterdam, New York (1978).

[13] X. Blanc, R. Costaouec, C. Le Bris and F. Legoll, Variance reduction in stochastic homogenization using antithetic variables. Markov Processes and Related Fields 18 (2012) 31-66. (preliminary version available at http://cermics.enpc.fr/ legoll/ hdr/FL24.pdf).

[14] X. Blanc, C. Le Bris and P.-L. Lions, Une variante de la théorie de l'homogénéisation stochastique des opérateurs elliptiques [A variant of stochastic homogenization theory for elliptic operators]. C.R. Acad. Sci. Sér. I 343 (2006) 717-724.

[15] X. Blanc, C. Le Bris and P.-L. Lions, Stochastic homogenization and random lattices. J. Math. Pures Appl. 88 (2007) 34-63.

[16] A. Bourgeat and A. Piatnitski, Estimates in probability of the residual between the random and the homogenized solutions of one-dimensional second-order operator. Asymptot. Anal. 21 (1999) 303-315.

[17] L. Carballal Perdiz, Etude d'une méthodologie multiéchelles appliquée à différents problèmes en milieu continu et discret (in french). Thèse de l'Université Toulouse III (2010). Available at http://thesesups.ups-tlse.fr/1170/.

[18] Z. Chen, Multiscale methods for elliptic homogenization problems, Numer. Methods Partial Differ. Eq. 22 (2006) 317-360.

[19] Z. Chen, M. Cui, T. Y. Savchuk and X. Yu, The multiscale finite element method with nonconforming elements for elliptic homogenization problems. SIAM Multiscale Model. Simul. 7 (2008) 517-538.

[20] Z. Chen and T.Y. Hou, A mixed multiscale finite element method for elliptic problems with oscillating coefficients. Math. Comput. 72 (2002) 541-576.

[21] Z. Chen and T.Y. Savchuk, Analysis of the multiscale finite element method for nonlinear and random homogenization problems. SIAM J. Numer. Anal. 46 (2008) 260-279. 
[22] P.G. Ciarlet, The finite element method for elliptic problems. North-Holland (1978).

[23] D. Cioranescu and P. Donato, An introduction to homogenization. In vol. 17 of Oxford Lect. Ser. Math. Appl. The Clarendon Press, Oxford University Press, New York (1999).

[24] R. Costaouec, Asymptotic expansion of the homogenized matrix in two weakly stochastic homogenization settings. Appl. Math. Res. Express 2012 (2012) 76-104.

[25] R. Costaouec, C. Le Bris and F. Legoll, Approximation numérique d'une classe de problèmes en homogénéisation stochastique [Numerical approximation of a class of problems in stochastic homogenization]. C.R. Acad. Sci. Série I 348 (2010) 99-103.

[26] P. Dostert, Y.R. Efendiev and T.Y. Hou, Multiscale finite element methods for stochastic porous media flow equations and application to uncertainty quantification. Comput. Methods Appl. Mechanics Engrg. 197 (2008) 3445-3455.

[27] W. E and B. Engquist, The heterogeneous multiscale methods. Commun. Math. Sci. 1 (2003) 87-132.

[28] W. E and B. Engquist, The Heterogeneous Multiscale Method for homogenization problems, in Multiscale Methods in Science and Engineering, vol. 44, Lect. Notes Comput. Sci. Engrg. Springer, Berlin (2005) 89-110.

[29] W. E, B. Engquist, X. Li, W. Ren and E. Vanden-Eijnden, Heterogeneous multiscale methods: a review. Commun. Comput. Phys. 2 (2007) 367-450.

[30] Y.R. Efendiev and T.Y. Hou, Multiscale finite element methods: theory and applications, Surveys and tutorials in the applied mathematical sciences. Springer, New York (2009).

[31] Y.R. Efendiev, T.Y. Hou and V. Ginting, Multiscale finite element methods for nonlinear problems and their applications. Commun. Math. Sci. 2 (2004) 553-589.

[32] Y.R. Efendiev, T.Y. Hou and X.-H. Wu, Convergence of a nonconforming multiscale finite element method. SIAM J. Numer. Anal. 37 (2000) 888-910.

[33] FreeFEM, http://www.freefem.org

[34] D. Gilbarg and N. S. Trudinger, Elliptic partial differential equations of second order, reprint of the 1998 edn., Classics in Mathematics. Springer (2001).

[35] V. Ginting, A. Malqvist and M. Presho, A novel method for solving multiscale elliptic problems with randomly perturbed data. SIAM Multiscale Model. Simul. 8 (2010) 977-996.

[36] A. Gloria, An analytical framework for numerical homogenization. Part II: Windowing and oversampling. SIAM Multiscale Model. Simul. 7 (2008) 274-293.

[37] T.Y. Hou and X.-H. Wu, A multiscale finite element method for elliptic problems in composite materials and porous media. J. Comput. Phys. 134 (1997) 169-189.

[38] T.Y. Hou, X.-H. Wu and Z. Cai, Convergence of a multiscale finite element method for elliptic problems with rapidly oscillating coefficients. Math. Comput. 68 (1999) 913-943.

[39] T.Y. Hou, X.-H. Wu and Y. Zhang, Removing the cell resonance error in the multiscale finite element method via a PetrovGalerkin formulation. Commun. Math. Sci. 2 (2004) 185-205.

[40] V.V. Jikov, S.M. Kozlov and O.A. Oleinik, Homogenization of differential operators and integral functionals. Springer-Verlag (1994).

[41] C. Le Bris, Some numerical approaches for "weakly" random homogenization, Numerical Mathematics and Advanced Applications 2009, in Proc. of ENUMATH 2009. Edited by G. Kreiss et al. Springer Lect. Ser. Notes Comput. Sci. Engrg. (2010) $29-45$.

[42] C. Le Bris, F. Legoll and F. Thomines, Rate of convergence of a two-scale expansion for some weakly stochastic homogenization problems. Asymptot. Anal. 80 (2012) 237-267.

[43] F. Legoll and F. Thomines, On a variant of random homogenization theory: convergence of the residual process and approximation of the homogenized coefficients. ESAIM: M2AN 48 (2014) 347-386.

[44] A. Lozinski, Habilitation à Diriger des Recherches, Université Paul Sabatier, Toulouse (2010). Available at http://www.math.univ-toulouse.fr/.

[45] Y. Maday, Reduced basis method for the rapid and reliable solution of partial differential equations, in vol. III of Intern. Congress of Math., Eur. Math. Soc. Zürich (2006) 1255-1270.

[46] Y. Mittal, Limiting behavior of maxima in stationary Gaussian sequences. Ann. Probab. 2 (1974) $231-242$.

[47] G.C. Papanicolaou and S.R.S. Varadhan, Boundary value problems with rapidly oscillating random coefficients, in vol. 10 of Proc. Colloq. on Random Fields: Rigorous Results in Statistical Mechanics and Quantum Field Theory, 1979. Edited by J. Fritz, J.L. Lebaritz and D. Szasz. Colloquia Mathematica Societ. J. Bolyai, North-Holland (1981) 835-873.

[48] L. Tartar, Estimations of homogenized coefficients, in Topics in the mathematical modelling of composite materials, vol. 31 of Progr. Nonlinear Differ. Equ. Appl., edited by A. Cherkaev and R. Kohn. Birkhäuser (1987). 